\title{
Computable Categoricity of Trees of Finite Height
}

\author{
Steffen Lempp \\ Department of Mathematics \\ University of Wisconsin-Madison \\ Charles McCoy \\ Department of Mathematics \\ University of Wisconsin-Madison
}

Russell Miller
Department of Mathematics
Queens College - C.U.N.Y.

Reed Solomon

Department of Mathematics

University of Connecticut*

August 11, 2004

\begin{abstract}
We characterize the structure of computably categorical trees of finite height, and prove that our criterion is both necessary and sufficient. Intuitively, the characterization is easiest to express in terms of isomorphisms of (possibly infinite) trees, but in fact it is equivalent to a $\Sigma_{3}^{0}$-condition. We show that all trees which are not computably categorical have computable dimension $\omega$. Finally, we prove that for every $n \geq 1$ in $\omega$, there exists a computable tree of finite height which is $\Delta_{n+1}^{0}$-categorical but not $\Delta_{n}^{0}$-categorical.
\end{abstract}

*The first author was partially supported by NSF grant DMS-9732526 and by the Vilas Foundation of the University of Wisconsin. The second author was partially supported by a VIGRE grant to the University of Wisconsin. The third author was partially supported by a VIGRE postdoc under NSF grant number 9983660 to Cornell University. The fourth author was partially supported by an NSF postdoctoral fellowship. 


\section{Introduction}

Computability theorists have developed powerful techniques for studying computational properties of the natural numbers. Many of these techniques can be applied to more general algebraic structures once they are suitably coded into the natural numbers. In this article, we use tools from computability theory to study computational problems for trees of finite height.

We begin with some general definitions and background in computable model theory. Let $\mathcal{A}$ be a countable structure over a fixed computable language whose domain $|\mathcal{A}|$ is a subset of $\omega$. The degree of $\mathcal{A}$ is the Turing degree of the atomic diagram of $(\mathcal{A}, a)_{a \in|\mathcal{A}|}$. In particular, if the language is finite, then $\mathcal{A}$ is computable if $|\mathcal{A}|$ is a computable set and the interpretations of the function and relation symbols are all computable. Throughout this paper, we assume that all structures are coded into the natural numbers.

In computable model theory, one frequently works in a given class of countable algebraic structures such as abelian groups, partial orders, fields, or as in this paper, finite height trees. Any computable structure from one of these classes is isomorphic to infinitely many other computable structures. It may happen, however, that two computable structures are isomorphic, yet that the only isomorphisms between them are noncomputable (as maps from one domain to the other). If so, then these structures lie in distinct computable isomorphism classes of the isomorphism type of the structure. On the other hand, if there exists a computable function taking one structure isomorphically to the other, then the two structures lie in the same computable isomorphism class.

The computable dimension of a computable structure is the number of computable isomorphism classes of that structure. The most common computable dimensions are 1 and $\omega$, and many classes of algebraic structures are known to admit only these computable dimensions. The following theorem is a compilation of results due to Goncharov ([12]); Goncharov, Dzgoev ([13]); Goncharov, Lempp, Solomon ([14]); LaRoche ([22]); Metakides, Nerode ([23]); Nurtazin ([28]); and Remmel ([29], [30]).

Theorem 1.1 Computable structures in the following classes have computable dimension 1 or $\omega$ : algebraically closed fields, real closed fields, abelian groups, linear orders, Boolean algebras, and ordered abelian groups.

On the other hand, Goncharov ([11]) proved that for each $0<n \leq \omega$, there exist structures with computable dimension $n$. Since then, many classes of structures have been discovered which admit computable dimension $n$ for each $0<n \leq \omega$. The following examples come from Goncharov ([11]); Goncharov, Molokov, Romanovskii ([15]); Hirschfeldt, Khoussainov, Shore, Slinko ([16]); and Kudinov ([21]).

Theorem 1.2 For each $0<n \leq \omega$, there are computable structures in the following classes with computable dimension n: graphs, lattices, partial orders, nilpotent groups, and integral domains. 
There are many other natural computational questions that one can ask about the members of these algebraic classes. For example, is it possible for the computable dimension of $\mathcal{A}$ to change when a single constant is named? What are the possible degree spectra for a structure or for a relation on a structure within each class? The degree spectrum of $\mathcal{A}$ is the set of Turing degrees $\mathbf{d}$ for which there is an isomorphic copy of $\mathcal{A}$ of degree $\mathbf{d}$. The degree spectrum of a relation $U$ on $\mathcal{A}$ is the set of degrees $\mathbf{d}$ such that there is an isomorphic computable copy of $\mathcal{A}$ for which the image of $U$ in this copy has degree $\mathbf{d}$.

Hirschfeldt, Khoussainov, Shore and Slinko ([16]) gave highly effective coding methods which show that for the classes of structures from Theorem 1.2, any answer to the above questions which can occur in a countable model, can actually occur within these classes. More specifically, they show that for each of these classes and for each nontrivial countable structure $\mathcal{M}$, there is a structure $\mathcal{A}$ from that class such that

1. the degree spectrum of $\mathcal{M}$ is equal to the degree spectrum of $\mathcal{A}$,

2. the computable dimension of $\mathcal{M}$ is the same as the computable dimension of $\mathcal{A}$,

3. for each $x \in|\mathcal{M}|$, there is an $a \in|\mathcal{A}|$ such that $(\mathcal{M}, x)$ has the same computable dimension as $(\mathcal{A}, a)$,

4. for each $S \subset|\mathcal{M}|$, there is a $U \subset|\mathcal{A}|$ such that the degree spectrum of the relation $S$ with respect to $\mathcal{M}$ is the same as the degree spectrum of $U$ with respect to $\mathcal{A}$.

These results suggest that the algebraic structure on the members of these classes interacts in a trivial way with the computational structure in the sense that any "pathological" computational behavior which can occur in a countable model can actually occur within these classes of structures. For example, Slaman ([32]) and Wehner ([34]) independently proved that there is a computable model $\mathcal{M}$ whose degree spectrum contains all degrees except $\mathbf{0}$. Therefore, by the result above, there are graphs, lattices, and so on with this property.

Because Property (2) above fails for the classes in Theorem 1.1, the interaction between the algebraic structure on the members of these classes and their computational structure is nontrivial in the sense that the algebraic structure necessarily limits the types of computational behavior that can occur. It is therefore interesting to ask about how the algebraic structure and the computational properties interact. For example, Downey and Jockusch ([7]) showed that every low Boolean algebra has a computable copy. Therefore, it is not possible for a Boolean algebra to have a degree spectrum consisting of all degrees except $\mathbf{0}$. On the other hand, by Miller ([25]), there is a linear order which has copies in every $\Delta_{2}^{0}$ degree except 0. The question of whether a linear order can have a spectrum consisting of all degrees except 0 remains open. The reader is referred to [16] for a more detailed survey of similar results.

We would hope for a fine line separating the classes of structures which behave as in Theorem 1.1 and those which behave as in Theorem 1.2. In the class of groups, the fact that abelian groups fall in Theorem 1.1 and nilpotent groups fall in Theorem 1.2 gives a reasonably sharp distinction. To sharpen the difference further, we could weaken nilpotent 
groups to torsion free nilpotent groups (that is, nilpotent groups in which no element except the identity has finite order) and we could add structure to the abelian groups by making them ordered. In both cases, the classes retain their previous possible computable dimensions.

For ring structures and ordered structures, the story is quite different. There is a large gap between algebraically or real closed fields (Theorem 1.1) and integral domains (Theorem 1.2). The obvious open question is what are the possible computable dimensions for computable fields. For ordered structures, there is a gap between linear orderings and Boolean algebras (Theorem 1.1) and lattices and partial orderings (Theorem 1.2). Trees are one obvious class of structures which falls within this gap and therefore they are of particular interest. It is not immediately apparent whether one would expect trees to admit only limited coding, like linear orders, or to admit very general coding, like partial orders, with respect to computable dimension and the other properties mentioned above. Our main result says that with respect to computable dimension, they have limited behavior in that they must have computable dimension 1 or $\omega$. It would therefore be interesting to explore the answers to the other computational questions for trees.

In addition to proving that finite height trees must have dimension 1 or $\omega$, we give an algebraic characterization for when they have computable dimension 1 . If the computable dimension of $\mathcal{A}$ is 1 , we say that $\mathcal{A}$ is computably categorical. This notion is somewhat analogous to the concept of categoricity in ordinary model theory: a theory is categorical in a given power $\kappa$ if all models of the theory of power $\kappa$ are isomorphic. Computable categoricity, however, is a property of structures, not of theories: a computable structure $\mathcal{A}$ is computably categorical if every other computable structure which is isomorphic to $\mathcal{A}$ is computably isomorphic to $\mathcal{A}$.

A standard example of a categorical theory is the theory of dense linear orders without end points, which is categorical in power $\omega$. One proves this by taking two arbitrary countable dense linear orders and building an isomorphism between them by a back-and-forth construction. The same construction allows us to prove that the structure $\mathbb{Q}$ is computably categorical. (More formally, let $(\omega, \prec)$ be a computable linear order isomorphic to $(\mathbb{Q},<)$. Then $(\omega, \prec)$ is computably categorical.)

Characterizations of computable categoricity have been found for several types of structures. The following examples comes from Goncharov, Dzgoev ([13]); Goncharov, Lempp, Solomon ([14]); and Remmel ([29], [30]).

Theorem 1.3 The following equivalences for computable categoricity hold.

1. A computable linear order is computably categorical if and only if it has a finite number of pairs of adjacent elements.

2. A computable Boolean algebra is computably categorical if and only if it has a finite number of atoms.

3. A computable ordered abelian group is computably categorical if and only if it has finite rank. 
There are a number of natural generalizations for computable categoricity, two of which are important for this article. A computable structure $\mathcal{A}$ is relatively computably categorical if for every isomorphic (not necessarily computable) copy $\mathcal{B}$, there is an isomorphism between $\mathcal{A}$ and $\mathcal{B}$ which is computable from the degree of $\mathcal{B}$. It follows directly from this definition that any structure which is relatively computably categorical is also computably categorical. For linear orders and Boolean algebras, the notions of computable categoricity and relative computable categoricity coincide. However, this is not always the case. In general, computable categoricity does not imply relative computable categoricity without addition assumptions on the structures involved.

Theorem 1.4 (Kudinov ([20])) There is a computable $\mathcal{A}$ for which the $\Pi_{1}^{0}$ diagram is decidable and which is computably categorical but not relatively so.

By the following result of Goncharov, Kudinov's example is the best possible in terms of decidable fragments.

Theorem 1.5 (Goncharov ([8])) Let $\mathcal{A}$ be a computable structure for which the $\Pi_{2}^{0}$ diagram is decidable. Then, $\mathcal{A}$ is relatively computably categorical if and only if it is computably categorical.

In the present paper we consider computable trees of finite height, and develop a structural criterion for such trees which is equivalent to both computable categoricity and relative computable categoricity. There are a number of definitions for trees, but for our purposes, a tree consists of a universe $T$ with a strict partial order $\prec$ on $T$ such that for every $x \in T$, $\prec$ well-orders the set of $\prec$-predecessors of $x$ in $T$, and such that $T$ contains a least element under $\prec$ called the root. We view our trees as growing upward with the root $r$ at the base. A tree is computable if $T$ is a computable set and $\prec$ is a computable relation. Without loss of generality, we can restrict ourselves to trees whose domain is an initial segment of $\omega$. An index for $T$ is then an index for the characteristic function of $\prec$.

Because we are only concerned with trees of finite height, we can define the level of a node $x \in T$ by

$$
\operatorname{level}_{T}(x)=|\{y \in T: y \prec x\}| .
$$

A more formal definition sets the level of the root to be 0 and inductively defines level $T(x)=$ $\sup \left\{\operatorname{level}_{T}(y)+1: y \prec x\right\}$, thereby also covering the case of an element with infinitely many predecessors. The level of a node is not generally computable, but it can be approximated from below by the computable function $f(x, s)=|\{y<s: y \prec x\}|$ which is increasing in the variable $s$ and which has the property that for all $x \in T, \operatorname{level}_{T}(x)=\lim _{s} f(x, s)$. The height of $T$ is defined by

$$
\operatorname{ht}(T)=\sup _{x \in T}\left(\operatorname{level}_{T}(x)+1\right) .
$$

A path $\gamma$ through $T$ is a maximal linearly ordered subset of $T$. Thus, for a finite height tree, the height of $T$ is the greatest $n$ such that $T$ contains a path with $n$ elements. In a tree of 
finite height $n$, we say that a node is established if it lies on a path of length $n$, and (for computable trees) established at stage $s$ if it lies on a path of length $n$ which is contained in the approximation $T_{s}$ at stage $s$. (If a node is established at stage $s$, then at that stage we know what its level in $T$ must be, since no more predecessors of the node can appear at later stages.)

We define our structural criterion for computable categoricity by induction on the height of the tree.

Definition 1.6 Let $(T, \prec)$ be a tree of finite height, and $x$ a node of $T$, with immediate successors $\left\{x_{i}: i \in I\right\}$. Let $T\left[x_{i}\right]=\left\{y \in T: x_{i} \preceq y\right\}$. We say that $x$ is of strongly finite type if it satisfies the following conditions:

i. There are only finitely many isomorphism types in the set $\left\{T\left[x_{i}\right]: i \in I\right\}$, each of which is of strongly finite type; and

ii. For each $j$ and $k$ in $I$, if $T\left[x_{j}\right]$ embeds into $T\left[x_{k}\right]$, then either $T\left[x_{j}\right]$ and $T\left[x_{k}\right]$ are isomorphic, or the isomorphism type of $T\left[x_{k}\right]$ appears only finitely often in $\left\{T\left[x_{i}\right]: i \in\right.$ $I\}$.

$T$ itself is of strongly finite type if every node in $T$ is of strongly finite type, or equivalently, if the root node is of strongly finite type. (By part (i), it is also equivalent to require that every minimal $\omega$-branch point in $T$ be of strongly finite type.)

Notice that ht $\left(T\left[x_{i}\right]\right)<\operatorname{ht}(T)$ for every $i \in I$, so that the concept is well-defined for every tree of finite height. Also, finite trees are automatically of strongly finite type, having no $\omega$-branch points. We also have a weaker criterion.

Definition 1.7 Using the same notation, we say that $x$ is of finite type if it satisfies:

i. There are only finitely many isomorphism types in the set $\left\{T\left[x_{i}\right]: i \in I\right\}$, each of which is of finite type; and

ii. Every isomorphism type which appears infinitely often in the set $\left\{T\left[x_{i}\right]: i \in I\right\}$ is of strongly finite type; and

iii. For each $j$ and $k$ in $I$, if $T\left[x_{j}\right]$ embeds into $T\left[x_{k}\right]$, then either $T\left[x_{j}\right]$ and $T\left[x_{k}\right]$ are isomorphic, or the isomorphism type of $T\left[x_{j}\right]$ appears only finitely often in $\left\{T\left[x_{i}\right]: i \in\right.$ $I\}$, or the isomorphism type of $T\left[x_{k}\right]$ appears only finitely often in $\left\{T\left[x_{i}\right]: i \in I\right\}$.

$T$ itself is of finite type if every node in $T$ is of finite type. Again, this is equivalent to every minimal $\omega$-branch point being of finite type.

We can now state our main theorem.

Theorem 1.8 For a computable tree $(T, \prec)$ of finite height, the following are equivalent: 
1. $T$ is of finite type;

2. $T$ is computably categorical;

\section{T has finite computable dimension;}

4. $T$ is relatively computably categorical.

The proof of Theorem 1.8 is contained in Sections 2, 3 and 4. In Section 2, we show that finite type implies relative computable categoricity, which in turn implies computable categoricity. In Sections 3 and 4, we show that any tree which is not of finite type cannot be computably categorical, which in turn implies that it is not relatively computably categorical. The proof proceeds by induction, with Section 3 containing the base case and Section 4 containing the inductive argument. This establishes the equivalence of conditions 1, 2 and 4 . We also show, in Section 4, that if a computable tree does not have finite type, then it must have infinite computable dimension. This establishes the equivalence of condition 3 with the other conditions.

To our knowledge, this is the first example of a structural criterion for computable categoricity which needs to be defined by recursion. Notice, however, that this criterion only applies to trees of finite height. The following result handles the case of infinite height trees.

Theorem 1.9 (Miller ([24])) The computable dimension of every computable tree of infinite height is $\omega$ (regardless of whether or not the tree has $\omega$-branch points).

Together, Theorems 1.8 and 1.9 show that trees, like linear orders, cannot exhibit the behavior of the structures listed in Theorem 1.2. Chisholm ([4]) has some related unpublished work for intrinsically 1-computable trees. A computable tree $T$ is 1-computable if its $\Sigma_{1}^{0}$ diagram is computable and $T$ is intrinsically 1-computable if every computable copy of $T$ is 1-computable. Chisholm proved that every intrinsically 1-computable tree is computably categorical. Notice, however, that intrinsic 1-computability is a strong assumption for trees, as Chisholm also showed that every intrinsically 1-computable tree is intrinsically decidable. (That is, every computable copy of the tree is decidable.)

Once we know that there are computable trees of finite height with infinite computable dimension, it is natural to ask exactly how difficult it is to compute an isomorphism between arbitrary pairs of such trees. (For computable trees without the restriction to finite height, the isomorphism problem is $\Sigma_{1}^{1}$-complete as computable trees can be used to code arbitrary computable ordinals.) In Section 5 we begin to answer this question for finite height trees by showing that no degree $\mathbf{0}^{(\mathbf{n})}$ is capable of computing an isomorphism between every pair of isomorphic computable trees of finite height. More specifically, a computable structure $\mathcal{A}$ is called $\Delta_{n}^{0}$-categorical if for every computable $\mathcal{B}$ isomorphic to $\mathcal{A}$, there is a $\Delta_{n}^{0}$-isomorphism from $\mathcal{A}$ to $\mathcal{B}$. In this notation, $\Delta_{1}^{0}$-categoricity is equivalent to computable categoricity. In Section 5, we show the following theorem. 
Theorem 1.10 For every $n \geq 1$ there is a computable tree of finite height which is $\Delta_{n+1^{-}}^{0}$ categorical but not $\Delta_{n}^{0}$-categorical.

Another natural question to ask is how difficult it is to express the property " $T$ is computably categorical". On its face, our structural criterion is an analytic predicate of similar complexity to stating the definition of computable categoricity. However, Ash, Knight, Mannasse and Slaman ([3]) showed that for any computable language $\mathcal{L}$, a computable $\mathcal{L}$-structure is relatively computably categorical if and only if it has a formally $\Sigma_{1}^{0}$ Scott family. In Section 2 , we show that computable trees of finite height and finite type have formally $\Sigma_{1}^{0}$ Scott families consisting of finitary formulas. (In fact, any formally $\Sigma_{1}^{0}$ Scott family can be transformed into one consisting of finitary formulas.) It is known (see Proposition 6.10 in Ash, Knight $([2]))$ that the existence of such families is described by a $\Sigma_{3}^{0}$ condition. Therefore, since computable categoricity and relative computable categoricity coincide for trees of finite height, there is a $\Sigma_{3}^{0}$ predicate which expresses " $T$ is computably categorical". Theories are known to exist in which the property of computable categoricity is strictly more complex than $\Sigma_{3}^{0}$; we refer the reader to [35] for details.

It is important to note that our definition of tree is based on a partial order $\prec$. In other papers, "tree" is sometimes defined using the infimum function $\wedge$, where the infimum $x \wedge y$ of $x$ and $y$ is the $\prec$-maximal $z$ such that $z \preceq x$ and $z \preceq y$. One can define $\prec$ from $\wedge$ by $a \prec b$ if and only if $a \wedge b=a$. Therefore, the notions of tree in terms of $\wedge$ and $\prec$ are classically bi-interpretable, and if $(T, \wedge)$ is a computable tree, then so is the corresponding $(T, \prec)$. However, the computability of $\prec$ need not imply computability of $\wedge$. Therefore, by choosing a definition based on $\prec$ rather than $\wedge$, we consider a broader class of computable trees. In Section 6, we give a brief discussion and conjecture about criteria for computable categoricity of trees in the language of the infimum.

By a homomorphism of trees, we mean a map which respects the partial orders, but need not preserve infima. Similarly, an embedding is a one-to-one homomorphism $T \hookrightarrow T^{\prime}$. We will frequently use the equivalence relation $\equiv$ given by

$$
T \equiv T^{\prime} \Longleftrightarrow T \hookrightarrow T^{\prime} \hookrightarrow T
$$

A tree $\left(T^{\prime}, \prec^{\prime}\right)$ is a subtree of $(T, \prec)$ if $T^{\prime} \subseteq T$ and the inclusion map respects the partial orders. Thus the infimum of two elements in $T$ may not be the same as their infimum in $T^{\prime}$. (This broader notion of subtree is another reason for choosing a definition of tree based on $\prec$ rather than $\wedge$.) Also, the root of $T$ may be distinct from the root of $T^{\prime}$, as in the case of the subtrees $T[x]$, which we will consider frequently. If $x \in T$, then $T[x]=\{y \in T: x \preceq y\}$. The partial order on $T[x]$ is the restriction to $T[x]$ of the partial order $\prec$ on $T$. Therefore $T[x]$ is a subtree of $T$ with root $x$. If $x$ is an immediate successor of the root in $T$, then we refer to $T[x]$ as a successor tree (of the root) in $T$. We define the height of $T$ above $x$ by

$$
\operatorname{ht}_{x}(T)=\operatorname{ht}(T[x]) .
$$




\section{Relatively computably categorical trees}

In [8], Goncharov gave a syntactic condition which, under some extra hypotheses, is equivalent to computable categoricity. In [3] Ash, Knight, Mannasse, and Slaman proved that this condition is actually equivalent, with no extra hypotheses needed, to the stronger notion of relative computable categoricity. In fact, they proved a more general result which applies to relative $\alpha$-categoricity for any $\alpha<\omega_{1}^{C K}$. In order to understand these statements fully, one would need to know about computable infinitary formulas as defined by Ash. However, all formulas we will need in this paper are finitary. Thus we state the relevant definitions and results correctly and completely, but we provide some clarifying remarks.

Definition 2.1 Let $\mathcal{L}$ be a computable language, and let $\mathcal{A}$ be a computable $\mathcal{L}$-structure. A formally $\Sigma_{1}^{0}$ Scott family for $\mathcal{A}$ is a c.e. collection $\Theta$ of computable $\Sigma_{1}$ formulas (possibly infinitary) so that

1. there is a finite tuple $\vec{c}$ so that for any $\theta(\vec{x}) \in \Theta$, all of the parameters appearing in $\theta$ are among $\vec{c}$;

2. for each tuple $\vec{a} \in \mathcal{A}$ of distinct elements, there is a $\theta(\vec{x}) \in \Theta$ so that $\mathcal{A}=\theta(\vec{a})$;

3. for each $\theta \in \Theta$, each tuple $\vec{a} \in \mathcal{A}$ of distinct elements and each tuple $\vec{a}^{\prime} \in \mathcal{A}$ of distinct elements, if $\mathcal{A} \models \theta(\vec{a}) \wedge \theta\left(\vec{a}^{\prime}\right)$, then $(\mathcal{A}, \vec{a}) \cong\left(\mathcal{A}, \vec{a}^{\prime}\right)$.

Note that since we talk about a c.e. set of formulas, we must have some way of assigning code numbers to the computable formulas. Note also that any finitary existential formula is (logically equivalent to) a computable $\Sigma_{1}^{0}$ formula. Again, throughout this paper, we will deal only with finitary formulas.

Theorem 2.2 (Ash-Knight-Mannasse-Slaman [3]) Let $\mathcal{L}$ be a computable language, and let $\mathcal{A}$ be a computable $\mathcal{L}$-structure. Then $\mathcal{A}$ is relatively computably categorical iff $\mathcal{A}$ has a formally $\Sigma_{1}^{0}$ Scott family.

The forward direction of this result is difficult and requires a forcing construction. The other direction follows from a straightforward back-and-forth argument. In this section, we use the easy direction of Theorem 2.2 to show that a tree with finite type is relatively computably categorical, and thus computably categorical.

Definition 2.3 Using the same notation as the introduction, we say that $x$ has weakly finite type if there are only finitely many isomorphism types in the set $\left\{T\left[x_{i}\right]: i \geq 1\right\}$, each of which has weakly finite type. $T$ itself has weakly finite type if every $\omega$-branch point in $T$ has weakly finite type.

Definition 2.4 Let $T$ be a tree of finite height with root node $r$, and let $x \in T, x \neq r$. $T_{x}$ is defined to be $\left(\{z \in T: z\right.$ is not comparable to $\left.x\} \cup\{r\}, \prec_{T}\right)$. 
We need to prove a few facts about this operation.

Lemma 2.5 Let $T$ be a tree of finite height with root node $r$. Let $x, y \in T, x, y \neq r$, and $x, y$ incomparable.

1. $T_{x}$ is a tree.

2. $\left(T_{x}\right)_{y}=\left(T_{y}\right)_{x}$.

3. If $T$ has weakly finite type, then so does $T_{x}$.

Proof. 1. The ordering relation on $T_{x}$ is inherited from $T$, and the root node of $T$, by definition, is in $T_{x}$.

2. Since each tree inherits its relation from $T$, we need only show that the two trees have the same underlying set. Note that $r$ is a member of both trees. Let $z \neq r$ be a member of $\left(T_{x}\right)_{y}$. Then $z$ is incomparable with $x$, since it belongs to $T_{x}$. (The operation never adds elements to a tree, so $\left(T_{x}\right)_{y} \subseteq T_{x}$.) And by definition, $z$ is incomparable with $y$ in $T$, since the relation on $T_{x}$ is inherited from $T$. Consequently, $z \in\left(T_{y}\right)_{x}$. By symmetry, $\left(T_{x}\right)_{y}=\left(T_{y}\right)_{x}$.

3. We induct on the height of $T$. First note that the definition only applies to trees of height $\geq 2$. If $\operatorname{ht}(T)=2$, then $x$ is an immediate successor of $r$ with no successors, so the claim is obviously true.

Let $\operatorname{ht}(T)=n+1$. If $x$ is an immediate successor of $r$, then $T_{x}=T-T[x]$, which has weakly finite type. If $x$ is strictly above an immediate successor $r_{1}$ of $r$, then we must first understand exactly how $T_{x}$ looks. Let $\left(r_{1}^{i}\right)_{i \in I}$ be the immediate successors of $r_{1}$ in the tree $\left(T\left[r_{1}\right]\right)_{x}$. (The set $I$ might be finite or infinite.) Then the tree $T_{x}$ is as follows:

1. $T-T\left[r_{1}\right] \subseteq T_{x} ;$ and

2. for each $i \in I$, the tree $\left(\left(T\left[r_{1}\right]\right)_{x}\right)\left[r_{1}^{i}\right]$ is attached directly above $r$.

By induction, $\left(T\left[r_{1}\right]\right)_{x}$ has weakly finite type. Consequently, each $\omega$-branch point of $\left(T\left[r_{1}\right]\right)_{x}$ has only finitely many types directly above it. Therefore, there are only finitely many isomorphism types in the set $\left\{\left(\left(T\left[r_{1}\right]\right)_{x}\right)\left[r_{1}^{i}\right]: i \in I\right\}$. And so, if $r$ is an $\omega$-branch point in $T_{x}$, then it has weakly finite type. Moreover, since $\left(T\left[r_{1}\right]\right)_{x}$ has weakly finite type and $T$ has weakly finite type, all other $\omega$-branch points of $T_{x}$ have weakly finite type. Thus, $T_{x}$ has weakly finite type.

Based on part 2 of the preceding lemma, if $x, y$ are incomparable nodes in $T$, we write $T_{x, y}$ for $\left(T_{x}\right)_{y}=\left(T_{y}\right)_{x}$. One further piece of notation we use is to denote the isomorphism type of a tree $T[x]$ by ot $(T[x])$.

Lemma 2.6 If $T$ has weakly finite type, then the set $\{$ ot $(T[x]): x \in T\}$ is finite. 
Proof. We induct on the height of $T$. If ht $(T)=1$, then it's clear. Let ht $(T)$ be $n+1$, let $r$ be its root node and its immediate successors be members of the sequence $\left(r_{i}\right)_{i \in I}$. Whether $I$ is finite or infinite, the set $\left\{o t\left(T\left[r_{i}\right]\right): i \in I\right\}$ is finite, since $T$ has weakly finite type. Moreover, by the induction hypothesis, for each $i \in I$, the set $\left\{o t(T[z]): z \succ r_{i}\right\}$ is finite. Consequently, $\{o t(T[x]): x \in T\}=\{o t(T)\} \cup\left\{o t\left(T\left[r_{i}\right]\right): i \in I\right\} \cup \bigcup_{i \in I}\left\{o t(T[z]): z \succ r_{i}\right\}$ is finite. (Even if $I$ is infinite, there are only finitely many different sets in this union by the comments above.)

Based on Lemma 2.6, we could restate Definition 2.3 by saying the a finite height tree $T$ has weakly finite type if and only if it has finitely many orbits under automorphisms of $T$.

Lemma 2.7 If $T$ has weakly finite type and $T$ has root node $r$, then the set $\left\{\right.$ ot $\left.\left(T_{x}\right): x \neq r\right\}$ is finite.

Proof. We induct on the height of $T$. If $h t(T)=2$, then there is only one element in the set $\left\{\right.$ ot $\left.\left(T_{x}\right): x \neq r\right\}$.

Let $h t(T)=n+1$. Let the immediate successors of $r$ be members of the sequence $\left(r_{i}\right)_{i \in I}$; and let $r_{1}, \ldots, r_{k}$ be the successors so that for all $i \in I$, there is $1 \leq j \leq k$ with $T\left[r_{i}\right] \cong T\left[r_{j}\right]$. By induction, we know that for each $1 \leq j \leq k$, there are $p_{j} \in \mathbb{N}$ and $r_{j}^{1}, \ldots, r_{j}^{p_{j}} \succ r_{j}$ so that for each $x \succ r_{j}$, there is $1 \leq q \leq p_{j}$ with $\left(T\left[r_{j}\right]\right)_{x} \cong\left(T\left[r_{j}\right]\right)_{r_{j}^{q}}$. It is clear from the description of $T_{x}$ in the proof of Lemma 2.5 that the set $\left\{\right.$ ot $\left.\left(T_{r_{j}}\right): 1 \leq j \leq k\right\} \cup \bigcup_{1 \leq j \leq k}\left\{\right.$ ot $\left.\left(T_{r_{j}^{q}}\right): 1 \leq q \leq p_{j}\right\}$ is equal to $\left\{o t\left(T_{x}\right): x \neq r\right\}$.

The proof of the next two lemmas will use induction on the degree of $\omega$-branching, which we define formally below. Intuitively, $T$ has degree $m \omega$-branching if and only if the following two conditions hold: first, there is a chain of elements in $T$ which contains $m$ many elements which are $\omega$ branching and second, for any chain of elements in $T$, there are at most $m$ many elements in the chain which are $\omega$-branching.

Definition 2.8 Let $T$ be a finite height tree.

1. $T$ has degree $0 \omega$-branching iff it is finite;

2. $T$ has degree $n+1 \omega$-branching iff

a. $T$ does not have degree $n \omega$-branching; and

b. if $x$ is a minimal $\omega$-branch point of $T$ with immediate successors $r_{i}, i \geq 1$, then for each $i \geq 1$, there is $m \leq n$ so that $T\left[r_{i}\right]$ has degree $m \omega$-branching.

Lemma 2.9 If $T_{1}$ and $T_{2}$ have weakly finite type and $T_{1} \hookrightarrow T_{2}$, then there is a finite subtree $T_{1}^{\prime} \subseteq T_{1}$ such that $T_{1}^{\prime} \nrightarrow T_{2}$. 
Proof. We induct on the height of tree $T_{1}$. If $\operatorname{ht}\left(T_{1}\right)=1$, then $T_{1} \hookrightarrow T_{2}$.

Let $T_{1}$ have height $n+1$. We induct on the degree of $\omega$-branching in $T_{1}$. First, if the degree is 0 , then $T_{1}$ is finite, so $T_{1}^{\prime}=T_{1}$.

Let $T_{1}$ have degree $m+1 \omega$-branching. We induct on the height of $T_{2}$. If $\operatorname{ht}\left(T_{2}\right)=1$, then $T_{1}$ is infinite, but $T_{2}$ is finite, so the desired conclusion is clear.

Let $T_{2}$ have height $p+1$. We induct on the degree of $\omega$-branching in $T_{2}$. If the degree is 0 , then then $T_{1}$ is infinite, but $T_{2}$ is finite.

Let $T_{2}$ have degree $q+1 \omega$-branching.

Case 1: The root node $r$ of $T_{1}$ is finite branching with immediate successors $r_{1}, \ldots, r_{k}$. By induction on the height of $T_{1}$, for each $i \in\{1, \ldots, k\}$ and each $s \in T_{2}$, if $T_{1}\left[r_{i}\right] \nsucc T_{2}[s]$, then there is a finite subtree $\left(T_{1}\left[r_{i}\right]\right)^{s} \subseteq T_{1}\left[r_{i}\right]$ so that $\left(T_{1}\left[r_{i}\right]\right)^{s} \leftrightarrow T_{2}[s]$. Consequently, by Lemma 2.6, for each $i \in\{1, \ldots, k\}$, there is a single finite $T_{1}\left[r_{i}\right]^{\prime} \subseteq T_{1}\left[r_{i}\right]$ so that for all $s \in T_{2}$, if $T_{1}\left[r_{i}\right] \nrightarrow T_{2}[s]$, then $T_{1}\left[r_{i}\right]^{\prime} \nrightarrow T_{2}[s]$. (We may assume that $r_{i} \in T_{1}\left[r_{i}\right]^{\prime}$ for each $i \in\{1, \ldots, k\}$.)

Define $T_{1}^{\prime} \subseteq T_{1}$ as follows:

1. $r \in T_{1}^{\prime}$;

2. $T_{1}\left[r_{i}\right]^{\prime} \subseteq T_{1}^{\prime}$ for each $i \in\{1, \ldots, k\}$.

We claim that $T_{1}^{\prime} \hookrightarrow T_{2}$. Assume otherwise that $f: T_{1}^{\prime} \hookrightarrow T_{2}$. Then $f$ maps $r, r_{1}, \ldots, r_{k}$ to some $s, s_{1}, \ldots s_{k}$ so that

1. $s$ is below all of $s_{1}, \ldots, s_{k}$;

2. no two of $s_{1}, \ldots, s_{k}$ are comparable; and

3. $T_{1}\left[r_{i}\right]^{\prime} \hookrightarrow T_{2}\left[s_{i}\right]$ for all $1 \leq i \leq k$.

Consequently, by the way in which we defined each $T_{1}\left[r_{i}\right]^{\prime}$,

1. $s$ is below all of $s_{1}, \ldots, s_{k}$;

2. no two of $s_{1}, \ldots, s_{k}$ are comparable; and

3. $T_{1}\left[r_{i}\right] \hookrightarrow T_{2}\left[s_{i}\right]$ for all $1 \leq i \leq k$.

Therefore, $T_{1} \hookrightarrow T_{2}$, a contradiction.

Case 2: The root node $r$ is an $\omega$-branch node with immediate successors $r_{i}, i \geq 1$, where $r_{1}, r_{2}, \ldots, r_{k}, r_{k+1}, \ldots, r_{l}$ are such that

1. $T_{1}\left[r_{1}\right], \ldots, T_{1}\left[r_{k}\right]$ are all of the direct successor trees of $r$ whose isomorphism types occur finitely often directly above $r$; 
2. $T_{1}\left[r_{k+1}\right], \ldots, T_{1}\left[r_{l}\right]$ are direct successor trees of $r$ whose isomorphism types occur infinitely often directly above $r$;

3. for all $j, j^{\prime}$ with $k+1 \leq j<j^{\prime} \leq l, T_{1}\left[r_{j}\right] \not T_{1}\left[r_{j^{\prime}}\right]$; and

4. for all $i \geq 1$, there is $j \leq l$ so that $T_{1}\left[r_{i}\right] \cong T_{1}\left[r_{j}\right]$.

Case 2a: The root node $s$ of $T_{2}$ is an $\omega$-branch node of $T_{2}$ with immediate successors $s_{i}, i \geq$ 1 , where $s_{1}, s_{2}, \ldots s_{t}, s_{t+1}, \ldots, s_{u}$ have properties analogous to those of $r_{1}, \ldots, r_{k}, r_{k+1} \ldots, r_{l}$. Consider $T_{3} \subset T_{1}$ defined as follows:

1. $r \in T_{3}$;

2. $T_{1}\left[r_{i}\right] \subset T_{3}$ for each $1 \leq i \leq k$.

If $T_{3} \nrightarrow T_{2}$, then by induction on the degree of $\omega$-branching in $T_{1}$, there is a finite subtree $T_{1}^{\prime} \subseteq T_{3} \subseteq T_{1}$ so that $T_{1}^{\prime} \hookrightarrow T_{2}$. So assume that $T_{3} \hookrightarrow T_{2}$. Thus, it cannot be the case that each $T_{1}\left[r_{k+1}\right], \ldots, T_{1}\left[r_{l}\right]$ embeds into one of $T_{2}\left[s_{t+1}\right], \ldots, T_{2}\left[s_{u}\right]$. Otherwise, $T_{1} \hookrightarrow T_{2}$. $\left(T_{2}\right.$ with countably many more copies of each of $T_{2}\left[s_{t+1}\right], \ldots T_{2}\left[s_{u}\right]$ attached directly above $s$ is a tree isomorphic to $T_{2}$ itself.)

Let $T_{1}\left[r_{\alpha_{1}}\right], \ldots, T_{1}\left[r_{\alpha_{v}}\right]$ be a list of all the trees among $T_{1}\left[r_{1}\right], \ldots, T_{1}\left[r_{k}\right]$ which individually do not embed into any $T_{2}\left[s_{t+1}\right], \ldots, T_{2}\left[s_{u}\right]$. Similarly, let $T_{1}\left[r_{\beta_{1}}\right], \ldots, T_{2}\left[r_{\beta_{w}}\right]$ be a list of all those among $T_{1}\left[r_{k+1}\right], \ldots, T_{1}\left[r_{l}\right]$ with this property. Consider the tree $T_{1}^{*} \subseteq T_{1}$ defined as follows:

1. $r \in T_{1}^{*}$;

2. $T_{1}\left[r_{\alpha_{1}}\right], \ldots, T_{1}\left[r_{\alpha_{v}}\right] \subset T_{1}^{*}$

3. for each $i \in\{1, \ldots, w\}, T_{1}^{*}$ contains infinitely many copies of $T_{1}\left[r_{\beta_{i}}\right]$ directly above $r$.

This tree cannot be embedded into the subtree $\{s\} \cup T_{2}\left[s_{1}\right] \cup \cdots \cup T_{2}\left[s_{t}\right]$; otherwise $T_{1} \hookrightarrow T_{2}$. By induction on the degree of $\omega$-branching of $T_{2}$, there is a finite number $\mu$ and a tree $\hat{T}_{1}$ so that

1. $r \in \hat{T}_{1}$

2. $T_{1}\left[r_{\alpha_{1}}\right], \ldots, T_{1}\left[r_{\alpha_{v}}\right] \subset \hat{T}_{1}$

3. for each $i \in\{1, \ldots, w\}, \hat{T}_{1}$ contains exactly $\mu$ copies of $T_{1}\left[r_{\beta_{i}}\right]$ directly above $r$; and

4. $\hat{T}_{1} \nrightarrow\{s\} \cup T_{2}\left[s_{1}\right] \cup \cdots \cup T_{2}\left[s_{t}\right]$. 
In fact, $\hat{T}_{1} \hookrightarrow T_{2}$. Why? Assume otherwise that $f: \hat{T}_{1} \hookrightarrow T_{2}$. None of the roots of the copies of $T_{1}\left[r_{\alpha_{1}}\right], \ldots, T_{1}\left[r_{\alpha_{v}}\right], T_{1}\left[r_{\beta_{1}}\right], \ldots, T_{2}\left[r_{\beta_{w}}\right]$ can be mapped to a point in $T_{2}\left[s_{i}\right]$ with $i \geq t+1$. Thus, $f: \hat{T}_{1} \hookrightarrow\{s\} \cup T_{2}\left[s_{1}\right] \cup \cdots \cup T_{2}\left[s_{t}\right]$, a direct contradiction. And so, by induction on the degree of $\omega$-branching of $T_{1}$, there is a finite subtree $T_{1}^{\prime} \subseteq \hat{T}_{1} \subset T_{1}$ such that $T_{1}^{\prime} \nrightarrow T_{2}$.

Case 2b: The root node $s$ of $T_{2}$ has finitely many immediate successors. We proceed by induction on the number of immediate successors $s$ has. First assume that $s$ has only one immediate successor $s_{1}$. Since the root node $r$ of $T_{1}$ has infinitely many immediate successors, we know, by induction on the height of $T_{2}$, that there is a finite tree $T_{1}^{\prime} \subset T_{1}$ so that

1. $r \in T_{1}^{\prime}$;

2. $r$ has at least two immediate successors in $T_{1}^{\prime}$; and

3. $T_{1}^{\prime} \nrightarrow T_{2}-\left\{s_{1}\right\}\left(\cong T_{2}\left[s_{1}\right]\right)$.

Of course, this implies that $T_{1}^{\prime} \hookrightarrow T_{2}$; if $f: T_{1}^{\prime} \hookrightarrow T_{2}$, then $f(r) \in T_{2}\left[s_{1}\right]$ or $s_{1} \notin \operatorname{range}(() f)$, since $s_{1}$ is the sole immediate successor of $s$, and $r$ has at least two.

Assume that $s$ has $t+1$ immediate successors $s_{1}, \ldots, s_{t+1}$ in $T_{2}$. Of course, for each $j \in\{1, \ldots, t+1\}, T_{1} \nrightarrow T_{2}-T_{2}\left[s_{j}\right]$. Therefore, by induction on the number of immediate successors of the root node $s$, for each $j \in\{1, \ldots, t+1\}$, there is a tree $\left(T_{1}\right)^{j} \subset T_{1}$ and a finite number $\mu_{j}$ so that

1. $r \in\left(T_{1}\right)^{j}$

2. for each $i \in\{1, \ldots, k\}, T_{1}\left[r_{i}\right] \subset\left(T_{1}\right)^{j}$;

3. for each $i \in\{k+1 \ldots, l\},\left(T_{1}\right)^{j}$ contains exactly $\mu_{j}$ many copies of $T_{1}\left[r_{i}\right]$ directly above $r$; and

4. $\left(T_{1}\right)^{j} \nrightarrow T_{2}-T_{2}\left[s_{j}\right]$.

Let $\mu=\max \left\{\mu_{j}: j \in\{1, \ldots, t+1\}\right\}$. Define $T_{1}^{*}$ as follows:

1. $r \in T_{1}^{*}$;

2. for each $i \in\{1, \ldots, k\}, T_{1}\left[r_{i}\right] \subset T_{1}^{*}$;

3. for each $i \in\{k+1 \ldots, l\}, T_{1}^{*}$ contains exactly $\mu$ many copies of $T_{1}\left[r_{i}\right]$ directly above $r$.

Assume, without loss of generality, that the immediate successors of $r$ in $T_{1}^{*}$ can be listed as $r_{1}, \ldots, r_{w}$. If for some $v_{1}, \ldots, v_{w} \in T_{2}, f: T_{1}^{*} \hookrightarrow T_{2}$ and $f\left(r_{i}\right)=v_{i}$ for $1 \leq i \leq w$, then the following must be true: 
a. for each $1 \leq j \leq t+1$, there is $1 \leq i \leq w$ so that $v_{i} \succeq s_{j}$; and

b. $\left(T_{1}-T_{1}^{*}\right) \cup\{r\} \hookrightarrow\left(T_{2}\right)_{v_{1}, \ldots, v_{w}}$ (where $\left(T_{2}\right)_{v_{1}, \ldots, v_{w}}$ is defined in Definition 2.5).

Otherwise, $\left(T_{1}\right)^{j} \hookrightarrow T_{2}-T_{2}\left[s_{j}\right]$ for some $j$ or $T_{1} \hookrightarrow T_{2}$.

Notice that if $v_{1}, \ldots, v_{w}$ satisfy a. above, then $\mathrm{ht}\left(\left(T_{2}\right)_{v_{1}, \ldots, v_{w}}\right)<\mathrm{ht}\left(T_{2}\right)$. And so, by induction on the height of tree $T_{2}$, it must be the case that for each tuple $\vec{v}=v_{1}, \ldots, v_{w} \in T_{2}$ which satisfy a. and b. above, there is a finite number $\kappa_{\vec{v}}$ and a subtree $T^{\vec{v}} \subseteq\left(T_{1}-T_{1}^{*}\right) \cup\{r\}$ so that

1. $r \in T^{\vec{v}}$

2. for each $i \in\{k+1, \ldots, l\}, T^{\vec{v}}$ contains exactly $\kappa_{\vec{v}}$ many copies of $T_{1}\left[r_{i}\right]$ directly above $r$; and

3. $T^{\vec{v}} \nrightarrow\left(T_{2}\right)_{v_{1}, \ldots, v_{w}}$.

Therefore, by Lemma 2.7, there is a single number $\kappa$ and a single tree $\hat{T} \subseteq\left(T_{1}-T_{1}^{*}\right) \cup\{r\}$ so that

1. $r \in \hat{T}$

2. for each $i \in\{k+1, \ldots, l\}, \hat{T}$ contains exactly $\kappa$ many copies of $T_{1}\left[r_{i}\right]$ directly above $r$; and

3. for each list of incomparable elements $v_{1}, \ldots, v_{w}$ which satisfy a. and b. above, $\hat{T} \hookrightarrow$ $\left(T_{2}\right)_{v_{1}, \ldots, v_{w}}$.

Finally, define $\hat{T}_{1} \subseteq T_{1}$ as follows:

1. $r \in \hat{T}_{1}$;

2. for each $i \in\{1, \ldots, k\}, T_{1}\left[r_{i}\right] \subset \hat{T}_{1}$;

3. for each $i \in\{k+1, \ldots, l\}, \hat{T}_{1}$ contains exactly $\mu+\kappa$ many copies of $T_{1}\left[r_{i}\right]$ directly above $r$.

A straightforward argument similar to ones previously given shows that $\hat{T}_{1} \hookrightarrow T_{2}$. Therefore, by induction on the degree of $\omega$-branching in $T_{1}$ it must be the case that there is a finite $T_{1}^{\prime} \subseteq \hat{T}_{1} \subset T_{1}$ so that $T_{1}^{\prime} \nrightarrow T_{2}$.

Lemma 2.10 If $T_{1}$ and $T_{2}$ have strongly finite type, and $T_{1} \hookrightarrow T_{2} \hookrightarrow T_{1}$, then $T_{1} \cong T_{2}$. 
This lemma need not hold if either $T_{1}$ or $T_{2}$ is only of finite type. For instance, let $T_{1}$ be the tree $\omega^{<3}$, with $x \preceq y$ iff $x$ is an initial segment of $y$, and delete $\{\langle 0, n\rangle: n \in \omega\}$ from $T_{1}$ to get $T_{2}$ (or let $T_{2}$ be any other tree of height 3 in which $T_{1}$ embeds). Recall that the equivalence relation $\equiv$ on all trees is defined by

$$
T \equiv T^{\prime} \Longleftrightarrow T \hookrightarrow T^{\prime} \hookrightarrow T
$$

So the lemma says that for trees of strongly finite type, $\equiv$ and $\cong$ are identical.

Proof. We induct on the height of $T_{1}$. If $\operatorname{ht}\left(T_{1}\right)=1$, then the result is obvious.

Let $\operatorname{ht}\left(T_{1}\right)=n+1$. We induct on the degree of $\omega$-branching of $T_{1}$. If the degree is 0 , then $T_{1}$ is finite. Therefore, if $T_{1} \hookrightarrow T_{2} \hookrightarrow T_{1}$, then both trees are finite of the same size, so any embedding must be an isomorphism.

Let the $T_{1}$ have degree $q+1 \omega$-branching. Let $r$ be the root node of $T_{1}$ and $s$ be the root of $T_{2}$. It is clear that $\mathrm{ht}\left(T_{1}\right)=\mathrm{ht}\left(T_{2}\right)$, and that in any embedding of one into the other, the root node must be mapped to the root node.

Case 1: $r$ has finitely many immediate successors. First, we argue that $s$ must have the same number of immediate successors. Let $r$ have immediate successors $r_{1}, \ldots, r_{m}$. Let $p$ be the number of trees among $T_{1}\left[r_{1}\right], \ldots, T_{1}\left[r_{m}\right]$ which have height equal to $n$. Since $T_{1} \hookrightarrow T_{2} \hookrightarrow T_{1}$, the number of such immediate successors of $s$ must also be $p$, and in the embeddings, a successor at the base of a tree of height $n$ must be mapped to a successor at the base of a tree of height $n$.

If $r$ has no more immediate successors, then $s$ cannot either, because $T_{2} \hookrightarrow T_{1}$. Otherwise, let $h_{1}$ be the greatest number $<n$ so that $r$ has an immediate successor $r_{i}$ with ht $\left(T_{1}\left[r_{i}\right]\right)=h_{1}$, and let $p_{1}$ be the number of such $r_{i}$ 's. First, notice that $s$ cannot have a successor $s_{i}$ with $h_{1}<$ $\operatorname{ht}\left(T_{2}\left[s_{i}\right]\right)<n$, since $T_{2} \hookrightarrow T_{1}$, and all of the successors $r_{i}$ of $r$ with $h t\left(T_{1}\left[r_{i}\right]\right)>h_{1}$ are images of successors $s_{i}$ of $s$ with ht $\left(T_{2}\left[s_{i}\right]\right)=n$. Moreover, $s$ must have exactly $p_{1}$ immediate successors $s_{i}$ with ht $\left(T_{2}\left[s_{i}\right]\right)=h_{1}$, since $T_{1} \hookrightarrow T_{2} \hookrightarrow T_{1}$. Continuing in this fashion we complete the argument that $r$ and $s$ must have exactly the same number of immediate successors.

We complete this case by arguing by induction on the number of immediate successors of $r, s$. First, if $r$ has only one immediate successor $r_{1}$, and $s$ has only one immediate successor $s_{1}$, then obviously, $T_{1}\left[r_{1}\right] \hookrightarrow T_{2}\left[s_{1}\right] \hookrightarrow T_{1}\left[r_{1}\right]$. By induction on the height on the trees, $T_{1}\left[r_{1}\right] \cong T_{2}\left[s_{1}\right]$, so $T_{1} \cong T_{2}$.

Assume that $r$ has immediate successors $r_{1}, \ldots, r_{m+1}$ and $s$ has immediate successors $s_{1}, \ldots, s_{m+1}$. By induction on the height of the trees, there must be a successor $r_{i}$ of $r$ so that

1. $T_{1}\left[r_{i}\right]$ has height $n$; and

2. $T_{1}\left[r_{i}\right]$ is maximal among $T_{1}\left[r_{1}\right], \ldots, T_{1}\left[r_{m+1}\right]$ with respect to embedding; i.e., if $j \neq i$ and $T_{1}\left[r_{i}\right] \hookrightarrow T_{1}\left[r_{j}\right]$, then $T_{1}\left[r_{i}\right] \cong T_{1}\left[r_{j}\right]$.

Assume, without loss of generality, that it is $r_{1}$. 
Now consider $f: T_{1} \hookrightarrow T_{2}$ and $g: T_{2} \hookrightarrow T_{1}$. Since $T_{1}\left[r_{1}\right]$ has height $n$, there are $1 \leq j, k \leq m+1$ such that $f: T_{1}\left[r_{1}\right] \hookrightarrow T_{2}\left[s_{j}\right]$ and $f: T_{1}-T_{1}\left[r_{1}\right] \hookrightarrow T_{2}-T_{2}\left[s_{j}\right]$; and $g: T_{2}\left[s_{j}\right] \hookrightarrow T_{1}\left[r_{k}\right]$ and $g: T_{2}-T_{2}\left[s_{j}\right] \hookrightarrow T_{1}-T_{1}\left[r_{k}\right]$. However, then $T_{1}\left[r_{1}\right] \hookrightarrow T_{1}\left[r_{k}\right]$, so by our choice of $T_{1}\left[r_{1}\right]$, we know that $T_{1}\left[r_{1}\right] \cong T_{1}\left[r_{k}\right]$. Consequently, by induction on the height of trees, $T_{1}\left[r_{1}\right] \cong T_{2}\left[s_{j}\right]$; and by induction on the number of immediate successors the root node has, $T_{1}-T_{1}\left[r_{1}\right] \cong T_{2}-T_{2}\left[s_{j}\right]$. Therefore, $T_{1} \cong T_{2}$.

Case 2: $r$ is an $\omega$-branch node with immediate successors $r_{i}, i \geq 1$, and $s$ is an $\omega$-branch node with immediate successors $s_{i}, i \geq 1$. First, consider the subtree $T_{1}^{\prime} \subseteq T_{1}$ which is defined as follows:

1. $r \in T_{1}^{\prime}$

2. $T_{1}\left[r_{i}\right] \subseteq T_{1}^{\prime}$ iff $T_{1}\left[r_{i}\right]$ has height $n$.

Define $T_{2}^{\prime}$ similarly. Of course $T_{1}^{\prime} \hookrightarrow T_{2}^{\prime} \hookrightarrow T_{1}^{\prime}$.

Next, consider the subtree $T_{1}^{\prime \prime} \subseteq T_{1}^{\prime}$ defined as follows:

1. $r \in T_{1}^{\prime \prime}$;

2. $T_{1}\left[r_{i}\right] \subseteq T_{1}^{\prime \prime}$ iff $T_{1}\left[r_{i}\right] \subseteq T_{1}^{\prime}$ and the isomorphism type of $T_{1}\left[r_{i}\right]$ occurs only finitely often directly above $r$.

Define $T_{2}^{\prime \prime}$ similarly. We claim that $T_{1}^{\prime \prime} \hookrightarrow T_{2}^{\prime \prime} \hookrightarrow T_{1}^{\prime \prime}$. To see this, let $T_{1}\left[r_{j}\right] \subset T_{1}^{\prime \prime}$. Of course, it must be the case that $T_{1}\left[r_{j}\right] \hookrightarrow T_{2}\left[s_{k}\right]$ for some $T_{2}\left[s_{k}\right] \subset T_{2}^{\prime}$. If $T_{2}\left[s_{k}\right] \not \subset T_{2}^{\prime \prime}$, then the isomorphism type of $T_{2}\left[s_{k}\right]$ occurs infinitely often directly above $s$. Since $h t\left(T_{2}\left[s_{k}\right]\right)=n$, it must be the case that $T_{2}\left[s_{k}\right] \hookrightarrow T_{1}\left[r_{l}\right]$, where the isomorphism type of $T_{1}\left[r_{l}\right]$ occurs infinitely often directly above $r_{0}$. However, then $T_{1}\left[r_{j}\right] \hookrightarrow T_{1}\left[r_{l}\right]$, a contradiction to the fact that $T_{1}$ has strongly finite type. And so, $T_{2}\left[s_{k}\right] \subset T_{2}^{\prime \prime}$. Consequently, $T_{1}^{\prime \prime} \hookrightarrow T_{2}^{\prime \prime}$. A symmetric argument shows that $T_{2}^{\prime \prime} \hookrightarrow T_{1}^{\prime \prime}$. By induction on the degree of $\omega$-branching of $T_{1}, T_{1}^{\prime \prime} \cong T_{2}^{\prime \prime}$.

Next, let $T_{1}\left[r_{j}\right] \subset T_{1}^{\prime}-T_{1}^{\prime \prime}$. Of course, it must be the case that $T_{1}\left[r_{j}\right] \hookrightarrow T_{2}\left[s_{k}\right]$ for some $T_{2}\left[s_{k}\right] \subset T_{2}^{\prime}-T_{2}^{\prime \prime}$. Similarly, $T_{2}\left[s_{k}\right] \hookrightarrow T_{2}\left[r_{l}\right]$ for some $T_{1}\left[r_{l}\right] \subset T_{1}^{\prime}-T_{1}^{\prime \prime}$. But then $T_{1}\left[r_{j}\right] \hookrightarrow T_{1}\left[r_{l}\right]$, so $T_{1}\left[r_{j}\right] \cong T_{1}\left[r_{l}\right]$, since $T_{1}$ has strongly finite type. By induction on the height of the trees, $T_{1}\left[r_{j}\right] \cong T_{1}\left[s_{k}\right]$. Similarly, let $T_{2}\left[s_{p}\right] \subset T_{2}^{\prime}-T_{2}^{\prime \prime}$. Then $T_{2}\left[s_{p}\right] \cong T_{1}\left[r_{t}\right]$ for some $T_{1}\left[r_{t}\right] \subset T_{1}^{\prime}-T_{1}^{\prime \prime}$. Therefore, $\{r\} \cup\left(T_{1}^{\prime}-T_{1}^{\prime \prime}\right) \cong\{s\} \cup\left(T_{2}^{\prime}-T_{2}^{\prime \prime}\right)$. Consequently, $T_{1}^{\prime} \cong T_{2}^{\prime}$.

Finally, we claim that if $f: T_{1} \hookrightarrow T_{2}$, then $f:\{r\} \cup\left(T_{1}-T_{1}^{\prime}\right) \hookrightarrow\{s\} \cup\left(T_{2}-T_{2}^{\prime}\right)$. Let $T_{1}\left[r_{j}\right] \nsubseteq T_{1}^{\prime}$, and let $f: T_{1}\left[r_{j}\right] \hookrightarrow T_{2}\left[s_{k}\right]$. It cannot be the case that $T_{2}\left[s_{k}\right] \subseteq T_{2}^{\prime \prime}$, because, as we have seen, the number of immediate successors $y$ of $s$ with $T_{2}[y] \subseteq T_{2}^{\prime \prime}$ is exactly the same as the number of immediate successors $x$ of $r$ with $T_{1}[x] \subseteq T_{1}^{\prime \prime}$, and each such $x$ must be sent to such an $y$. Also, it cannot be the case that $T_{2}\left[s_{k}\right] \subseteq T_{2}^{\prime}-T_{2}^{\prime \prime}$; otherwise, as we have seen, it would be the case that $T_{2}\left[s_{k}\right] \cong T_{1}\left[r_{l}\right]$ where $T_{1}\left[r_{l}\right]$ occurs infinitely often immediately above $r$; but $T_{1}\left[r_{j}\right] \hookrightarrow T_{1}\left[r_{l}\right]$, since $T_{1}$ has strongly finite type. Thus $f: T_{1}\left[r_{j}\right] \hookrightarrow T_{1}\left[s_{k}\right]$, where $T_{1}\left[s_{k}\right] \subseteq T_{1}-T_{1}^{\prime}$. Therefore, $f:\{r\} \cup\left(T_{1}-T_{1}^{\prime}\right) \hookrightarrow\{s\} \cup\left(T_{2}-T_{2}^{\prime}\right)$. A symmetric argument shows that $\{s\} \cup\left(T_{2}-T_{2}^{\prime}\right) \hookrightarrow\{r\} \cup\left(T_{1}-T_{1}^{\prime}\right)$. Therefore, by induction on the height of the trees, $\{r\} \cup\left(T_{1}-T_{1}^{\prime}\right) \cong\{s\} \cup\left(T_{2}-T_{2}^{\prime}\right)$. And so, $T_{1} \cong T_{2}$. 
Lemma 2.11 If $T$ has strongly finite type, then $T$ has a formally $\Sigma_{1}^{0}$ Scott family of finitary formulas with no parameters.

Proof. First, note that if $T$ is a tree and $T^{\prime}$ is a finite tree, then we can say that $T^{\prime}$ can be embedded in $T[x]$ with a finitary existential formula $\psi(x)$.

We induct on the height of tree $T$. Let $r$ be the root node of $T$. If $\operatorname{ht}(T)=1$, then $T=\{r\}$, so $\{x=x\}$ is the desired Scott family.

Let $\operatorname{ht}(T)=n+1$. Let $r$ have immediate successors $\left(r_{i}\right)_{i \in I}$. First, let $r_{1}, \ldots, r_{k}, r_{k+1}, \ldots, r_{l}$ be such that

1. $T_{1}\left[r_{1}\right], \ldots, T_{1}\left[r_{k}\right]$ are all of the direct successor trees of $r$ whose isomorphism types occur finitely often directly above $r$;

2. $T_{1}\left[r_{k+1}\right], \ldots, T_{1}\left[r_{l}\right]$ are direct successor trees of $r$ whose isomorphism types occur infinitely often directly above $r$ (this list is empty if $I$ is finite);

3. for all $j, j^{\prime}$ with $k+1 \leq j<j^{\prime} \leq l, T_{1}\left[r_{j}\right] \varsubsetneqq T_{1}\left[r_{j^{\prime}}\right]$; and

4. for all $i \in I$, there is $j \leq l$ so that $T_{1}\left[r_{i}\right] \cong T_{1}\left[r_{j}\right]$.

Next, for each $j \in\{1, \ldots, l\}$, let $T_{j}^{\prime}$ be such that

1. $T_{j}^{\prime} \subseteq T\left[r_{j}\right]$

2. $T_{j}^{\prime}$ is finite; and

3. for all $i \in I$, if $T\left[r_{j}\right] \nrightarrow T\left[r_{i}\right]$, then $T_{j}^{\prime} \nrightarrow T\left[r_{i}\right]$.

Finally, for each $1 \leq j \leq l$, let $\Psi_{j}$ be the Turing machine which enumerates the formally $\Sigma_{1}^{0}$ Scott family for $T\left[r_{j}\right]$.

Given a tuple of variables $\vec{x}=x_{1}, \ldots, x_{t}$ of length $t$, we consider all 7-tuples $\rho_{t}=$ $\left\langle a, j, s, P, \sigma, \tau, s^{\prime}\right\rangle$, where

1. $a \in\{0,1\}$ and $1 \leq j \leq t$ : if $a=0$, then no member of $\vec{x}$ is going to "represent" $r$; if $a=1$, then $x_{j}$ is going to "represent" the root node $r$.

2. $s \leq \max \{|I|, t-a\}, P$ is a partition of $\{1, \ldots, t\}(\{1, \ldots, t\}-\{j\}$ if $a=1)$ into $s$ pieces: for the part of $\vec{x}$ remaining, we divide it into subtuples $\vec{y}_{1}, \ldots, \vec{y}_{s}$ according to $P$.

3. $\sigma=\left\langle i_{1}, \ldots, i_{s}\right\rangle$ is an $s$-tuple so that
a. for each $1 \leq \mu \leq s, i_{\mu} \in\{1, \ldots, l\}$;
b. for each $1 \leq \mu \leq s,\left|\vec{y}_{\mu}\right| \leq\left|T\left[r_{i_{\mu}}\right]\right|$; and
c. for each $1 \leq \mu<\nu \leq s$, if $i_{\mu}=i_{\nu}$, then $i_{\mu} \in\{k+1, \ldots, l\}$. 
4. $\tau$ is an $s$-tuple of natural numbers: for each $1 \leq \mu \leq s, \tau(\mu)$ tells us which formula to use from the formally $\Sigma_{1}^{0}$ Scott family for $T\left[r_{i_{\mu}}\right]$.

5. $s^{\prime}$ is a natural number.

Now we form the formula $\gamma_{\rho_{t}}(\vec{x})$ :

1. If in 1. above, $a=1$, then $\gamma_{\rho_{t}}$ includes a conjunct which says that $x_{j}$ is at the bottom of a chain of length $n+1$; otherwise, $\gamma_{\rho_{t}}$ includes a conjunct which says that there is $v_{0}$ so that $v_{0} \prec \vec{x}$.

2. For each tuple $\vec{y}_{\mu}$ and each tree $T\left[r_{i_{\mu}}\right]$ from 2. and 3. above, $\gamma_{\rho_{t}}$ includes conjuncts $\gamma_{\mu}\left(\vec{y}_{\mu}\right)$ so that

a. if $j_{1}, \ldots, j_{w_{\mu}}$ is a complete list of the elements of $\{1, \ldots, k\}$ so that $T\left[r_{i_{\mu}}\right] \hookrightarrow$ $T\left[r_{j_{1}}\right], \ldots, T\left[r_{j_{w_{\mu}}}\right]$, but $T\left[r_{i_{\mu}}\right] \approx T\left[r_{j_{1}}\right], \ldots, T\left[r_{j_{w_{\mu}}}\right]$ (and thus Lemma 2.10 implies $\left.T\left[r_{j_{1}}\right], \ldots, T\left[r_{j_{w_{\mu}}}\right] \nrightarrow T\left[r_{i_{\mu}}\right]\right)$, then $\gamma_{\mu}\left(\vec{y}_{\mu}\right)$ includes the conjunct which says $\exists v_{0} v_{1} \cdots v_{w_{\mu}+1}\left[v_{0}\right.$ is at the bottom of a chain of length $n+1 ; v_{1}, \ldots, v_{w_{\mu}}, v_{w_{\mu}+1}$ are incomparable; for each $1 \leq p \leq w_{\mu}, T_{j_{p}}^{\prime} \hookrightarrow T\left[v_{p}\right]$, and $v_{p}$ is at the bottom of a chain of length ht $\left(T\left[r_{j_{p}}\right]\right) ; \vec{y}_{\mu} \succeq v_{w_{\mu}+1}$, and $T_{i_{\mu}}^{\prime} \hookrightarrow T\left[v_{w_{\mu}+1}\right]$;

b. if both of the following are true about $\Psi_{i_{\mu}}$ :

i. $\Psi_{i_{\mu}}$ halts on input $\tau(\mu)$ (a natural number) in less than $s^{\prime}$ steps and outputs a formula $\delta(\vec{z})$; and

ii. $\left|\vec{y}_{\mu}\right|$ is the number of free variables actually appearing in $\delta(\vec{z})$,

then $\gamma_{\mu}\left(\vec{y}_{\mu}\right)$ includes the conjunct $\delta\left(\vec{y}_{\mu}\right)$. Otherwise, it includes the conjunct $\perp$ (falsity).

3. For each $1 \leq \mu<\nu \leq s$ so that $i_{\mu} \neq i_{\nu}$, but $T\left[r_{i_{\mu}}\right] \cong T\left[r_{i_{\nu}}\right], \gamma_{\rho_{t}}(\vec{x})$ includes a conjunct which says $\exists v_{1} v_{2}\left[v_{1}\right.$ and $v_{2}$ are incomparable; each is at the bottom of a chain of length $\operatorname{ht}\left(T\left[r_{i_{\mu}}\right]\right) ; \vec{y}_{\mu} \succeq v_{1}$ and $\left.\vec{y}_{\nu} \succeq v_{2}\right]$.

Let $\Theta=\left\{\gamma_{\rho_{t}}(\vec{x}): t \in \omega, \vec{x}\right.$ is a $t$-tuple, and $\vec{x}, \rho_{t}$ are as above $\}$. We claim that $\Theta$ is a formally $\Sigma_{1}^{0}$ Scott family of the desired form. First, since we explicitly describe how to form the formulas $\gamma_{\rho_{t}}, \Theta$ is certainly c.e. Next, if $\vec{a}$ is a $t$-tuple of distinct elements of $T$, then it is obvious to see that there is going to be some $\gamma_{\rho_{t}}(\vec{x})$ which it satisfies. Finally, assume that $\vec{a}=a_{1}, \ldots, a_{t}$ and $\vec{b}=b_{1}, \ldots, b_{t}$ are two tuples of distinct elements so that $T \models \gamma_{\rho_{t}}(\vec{a}) \wedge \gamma_{\rho_{t}}(\vec{b})$. We must show that $(T, \vec{a}) \cong(T, \vec{b})$.

First, it must be the case that $\vec{a}$ contains the root node $r$ iff $\vec{b}$ does, and that $a_{j}=r$ iff $b_{j}=r$. Next, since $T \models \gamma_{\rho_{t}}(\vec{a}), \vec{a}$ (perhaps without $a_{j}$ ) is sorted into $\vec{a}_{1}, \vec{a}_{2}, \ldots, \vec{a}_{s}$ (according to the substitution of $\vec{a}_{\mu}$ for the subtuple of variables $\vec{y}_{\mu}$ for each $1 \leq \mu \leq s$ ) so that for each $1 \leq \mu \leq s$ the following things are determined: 
1. all elements in a single tuple $\vec{a}_{\mu}$ are contained in the same direct successor tree of $r$;

2. $\vec{a}_{\mu}$ belongs to a direct successor tree of $r$ of order type that of $T\left[r_{i_{\mu}}\right]$;

3. for all $\nu$ with $1 \leq \nu \leq s$ and $\nu \neq \mu$, some element of $\vec{a}_{\mu}$ and some element of $\vec{a}_{\nu}$ are not contained in the same direct successor tree of $r$; and

4. $\vec{a}_{\mu}$ satisfies the formula $\delta(\vec{z})$, obtained from the formally $\Sigma_{1}^{0}$ Scott family for $T\left[r_{i_{\mu}}\right]$.

To see 2., notice that clause a. in the formation of $\gamma_{\vec{y}_{\mu}}$ guarantees that $\vec{a}_{\mu}$ is contained in a direct successor tree of $r$ which embeds $T_{i_{\mu}}^{\prime}$, and hence $T\left[r_{i_{\mu}}\right]$. Therefore, we know that $\vec{a}_{\mu}$ is either contained in a direct successor tree of order type $T\left[r_{i_{\mu}}\right]$ or one of the finitely many non-isomorphic direct successor trees which embed $T\left[r_{i_{\mu}}\right]$. But the rest of clause a. rules out all other possibilities.

To see 3., assume that in 2. we have determined that the type of the direct successor tree to which $\vec{a}_{\mu}$ belongs is the same as the type of the direct successor tree to which $\vec{a}_{\nu}$ belongs. Then $\gamma_{\rho}(\vec{a})$ contains a conjunct which says that $\exists v_{1} v_{2}\left[v_{1}\right.$ and $v_{2}$ are incomparable; each of $v_{1}, v_{2}$ is at the bottom of a chain of length $\operatorname{ht}\left(T\left[r_{i_{\mu}}\right]\right)$; and $v_{1} \preceq \vec{a}_{\mu}$; and $\left.v_{2} \preceq \vec{a}_{\nu}\right]$.

Of course, the tuple $\vec{b}$ is sorted by $\gamma_{\rho_{t}}$ in exactly the same way. Therefore, by the definition of a Scott family, $r$ has immediate successor trees $T_{1}, \ldots, T_{s}, T_{1}^{\prime}, \ldots, T_{s}^{\prime}$ so that for all $1 \leq p \neq$ $q \leq s$ the following are true:

1. $T_{p} \neq T_{q}$, and $T_{p}^{\prime} \neq T_{q}^{\prime}$;

2. $\vec{a}_{p} \in T_{p} ; \vec{b}_{p} \in T_{p}^{\prime}$; and

3. $\left(T_{p}, \vec{a}_{p}\right) \cong\left(T_{p}^{\prime}, \vec{b}_{p}\right)$.

And so, $(T, \vec{a}) \cong(T, \vec{b})$.

Our next result shows that finite type implies relative computable categoricity in Theorem 1.8 .

Theorem 2.12 If $T$ has finite type, then $T$ has a formally $\Sigma_{1}^{0}$ Scott family of finitary formulas.

Proof. We induct on the height of $T$. If ht $(T)=1$, then $T$ is finite, so $T$ has strongly finite type, and the previous result applies. Let $\operatorname{ht}(T)=n+1$, and let $r$ be the root node of $T$.

Case 1: $r$ has only finitely many immediate successors $r_{1}, \ldots, r_{m}$. Then $T\left[r_{1}\right], T\left[r_{2}\right], \ldots, T\left[r_{m}\right]$ all have finite type, and hence all have formally $\Sigma_{1}^{0}$ Scott families by induction. For each $1 \leq i \leq m$, let $\vec{c}_{i}$ be the parameters of $T\left[r_{i}\right]$ which appear in the Scott family for $T\left[r_{i}\right]$. Let the tuple of parameters of our formally $\Sigma_{1}^{0}$ Scott family be $\vec{c}=r, r_{1}, \ldots, r_{m}, \vec{c}_{1}, \ldots, \vec{c}_{m}$.

Let $\vec{a}$ be a tuple of distinct elements in $T$, and let $\vec{x}$ be a corresponding tuple of variables. We construct the formula $\gamma_{\vec{a}}(\vec{x})$ as follows: 
1. if any $a_{i} \in \vec{a}$ is equal to an element $c$ of our parameter set, then we include the conjunct $x_{i}=c$;

2 . let $\vec{a}^{\prime}$ be the tuple $\vec{a}$ with such elements removed, and let $\vec{x}^{\prime}$ be the corresponding tuple of variables; we include a conjunct which says that $\vec{x}^{\prime} \cap \vec{c}=\emptyset$;

3. we divide the tuple $\vec{a}^{\prime}$ into subtuples $\vec{a}_{1}, \ldots, \vec{a}_{m}$ so that $\vec{a}_{i} \in T\left[r_{i}\right]$ (some subtuples might be empty); we divide the tuple of variables $\vec{x}^{\prime}$ into $\vec{x}_{1}, \ldots, \vec{x}_{m}$ accordingly; for each $1 \leq i \leq m$, we include the conjunct which says that $\vec{x}_{i} \succeq r_{i}$;

4. for each tuple $\vec{a}_{i}$, we search until we find the first formula $\delta_{i}$ from the formally $\Sigma_{1}^{0}$ Scott family for $T\left[r_{i}\right]$ which $\vec{a}_{i}$ satisfies; we include the conjunct $\delta_{i}\left(\vec{x}_{i}\right)$.

Let $\Theta=\left\{\gamma_{\vec{a}}(\vec{x}): \vec{a} \in T\right.$ is a tuple of distinct variables $\}$. It is clear that $\Theta$ is a formally $\Sigma_{1}^{0}$ Scott family of finitary formulas.

Case 2: $r$ has infinitely many immediate successors $r_{i}, i \geq 1$. As usual, let $r_{1}, \ldots, r_{k}, \ldots, r_{l}$ be such that

1. $T_{1}\left[r_{1}\right], \ldots, T_{1}\left[r_{k}\right]$ are all of the direct successor trees of $r$ whose isomorphism types occur finitely often directly above $r$;

2. $T_{1}\left[r_{k+1}\right], \ldots, T_{1}\left[r_{l}\right]$ are direct successor trees of $r$ whose isomorphism types occur infinitely often directly above $r$;

3. for all $j, j^{\prime}$ with $k+1 \leq j<j^{\prime} \leq l, T_{1}\left[r_{j}\right] \approx T_{1}\left[r_{j^{\prime}}\right]$; and

4. for all $i \geq 1$, there is $j \leq l$ so that $T_{1}\left[r_{i}\right] \cong T_{1}\left[r_{j}\right]$.

By induction, each of the trees $T\left[r_{1}\right], \ldots, T\left[r_{k}\right]$ has a formally $\Sigma_{1}^{0}$ Scott family of finitary formulas. For each $1 \leq i \leq k$, let $\vec{c}_{i}$ be the parameters of $T\left[r_{i}\right]$ which appear in the Scott family for $T\left[r_{i}\right]$. Furthermore, notice that that the tree $T-\bigcup_{1<i<k} T\left[r_{i}\right]$ has strongly finite type. Therefore, by the previous lemma, this tree has a formally $\Sigma_{1}^{0}$ Scott family of finitary formulas with no parameters. Let the tuple of parameters of our formally $\Sigma_{1}^{0}$ Scott family be $r, r_{1}, \ldots, r_{k}, \vec{c}_{1}, \ldots, \vec{c}_{k}$.

Let $\vec{a}$ be a tuple of distinct elements in $T$, and let $\vec{x}$ be a corresponding tuple of variables. We construct the formula $\gamma_{\vec{a}}(\vec{x})$ as follows:

1. if any $a_{i} \in \vec{a}$ is equal to an element $c$ of our tuple of parameters, then we include the conjunct $x_{i}=c$;

2. let $\vec{a}^{\prime}$ be the tuple $\vec{a}$ with such elements removed, and let $\vec{x}^{\prime}$ be the corresponding tuple of variables; we include a conjunct which says that $\vec{x}^{\prime} \cap \vec{c}=\emptyset$; 
3. we divide the tuple $\vec{a}^{\prime}$ into subtuples $\vec{a}_{1}, \ldots, \vec{a}_{k}, \vec{a}_{k+1}$ so that $\vec{a}_{i} \in T\left[r_{i}\right]$ for $1 \leq 1 \leq k$ and $\vec{a}_{k+1} \in T-\bigcup_{1<i<k} T\left[r_{i}\right]$ (some subtuples might be empty); we divide the tuple of variables $\vec{x}^{\prime}$ into $\vec{x}_{1}, \ldots, \vec{x}_{k}, \vec{x}_{k+1}$ accordingly; for each $1 \leq i \leq k$, we include the conjunct which says that $\vec{x}_{i} \succeq r_{i}$; for each element $a$ of $\vec{a}_{k+1}$, we include a conjunct which says that the corresponding variable $x$ is incomparable to $r_{1}, \ldots, r_{k}$;

4. for each $1 \leq i \leq k$, we search until we find the first formula $\delta_{i}$ from the formally $\Sigma_{1}^{0}$ Scott family for $T\left[r_{i}\right]$ which $\vec{a}_{i}$ satisfies; we include the conjunct $\delta_{i}\left(\vec{x}_{i}\right)$;

5. we search until we find a formula $\delta_{k+1}$ from the formally $\Sigma_{1}^{0}$ Scott family for $T-$ $\bigcup_{1 \leq i \leq k} T\left[r_{i}\right]$ which $\vec{a}_{k+1}$ satisfies; we include the conjunct $\delta_{k+1}\left(\vec{x}_{k+1}\right)$.

Let $\Theta=\left\{\gamma_{\vec{a}}(\vec{x}): \vec{a} \in T\right.$ is a tuple of distinct variables $\}$. It is clear that $\Theta$ is a formally $\Sigma_{1}^{0}$ Scott family of finitary formulas.

\section{Computably Non-Categorical Trees}

To prepare for the induction that establishes Theorem 1.8, we will prove the following:

Proposition 3.1 Let $T$ be a computable tree of finite height with root $r$. If $r$ is not of finite type but every other node in $T$ is of finite type, then $T$ is not computably categorical. Indeed, $T$ has computable dimension $\omega$.

Our proof of Proposition 3.1 requires several distinct finite-injury constructions for the different possible cases. In each construction we build a computable tree $T^{\prime}$ isomorphic to $T$ satisfying the requirements

$$
\mathcal{R}_{e}: \varphi_{e} \text { one-one and total } \Longrightarrow\left[\left(\exists w_{e} \in T\right) T\left[w_{e}\right] \not T^{\prime}\left[\varphi_{e}\left(w_{e}\right)\right]\right] \text {. }
$$

We guarantee that $T^{\prime}$ is isomorphic to $T$ by building a $\Delta_{2}^{0}$ function $f: T \rightarrow T^{\prime}$. $f$ will either be an isomorphism from $T$ onto $T^{\prime}$ or it will be an isomorphism from $T$ onto range $(f)$. In the latter case, $T^{\prime} \backslash$ range $(f)$ will consist of successor trees of the root in $T^{\prime}$ each of which will have the same isomorphism type as a successor tree of the root in $T$ which occurs infinitely often in $T$. Therefore, despite the extra successor trees, $T$ and $T^{\prime}$ will be isomorphic. Goncharov ([10]) proved that if two computable structures are not computably isomorphic but are $\Delta_{2}^{0}$ isomorphic, then their computable dimension is $\omega$. So, in the case where $f$ is an isomorphism, we get the infinite dimension part of the theorem for free. We make a separate argument at the end of the section for the case when $f$ does not map onto $T^{\prime}$.

The node $w_{e}$ will be called the witness node for requirement $\mathcal{R}_{e}$, and will be approximated by nodes $w_{e, s}$ at each stage $s$. At certain stages we will need to find embeddings of $T_{s}\left[w_{e, s}\right]$ into other branches of $T$, in order to satisfy $\mathcal{R}_{e}$, and we may redefine $f$ on the nodes in $T_{s}\left[w_{e, s}\right]$. 
(We assume that we work with a fixed approximation $T_{s}$ of $T$ by finite subtrees.) To ensure that $\lim _{s} f_{s}(x)$ exists for each $x \in T$, we impose the negative requirements:

$$
\mathcal{N}_{x}: \quad \lim _{s} f_{s}(x) \text { converges. }
$$

In addition, for any $y \in T^{\prime}$, we need to insure that either $\lim _{s} f_{s}^{-1}(y)$ exists or $y$ is permanently placed into one of the auxiliary subtrees of $T^{\prime}$ which are not in the range of $f$ but which occur infinitely often as successor trees of the root in $T$. In the cases when we use such auxiliary trees, this property will be easy to verify. In the other cases, we explicitly insure this property by meeting the requirements for all $u \in T^{\prime}$ :

$$
\mathcal{M}_{u}: \lim _{s} f_{s}^{-1}(u) \text { converges. }
$$

Clearly, satisfying all these requirements will prove the theorem.

By definition, a successor tree in $T$ above an $\omega$-branch point $x$ of $T$ is a tree $T[y]$, where $y$ is an immediate successor of $x$. We use $I$ to stand for an isomorphism type, and say that $I$ appears finitely often (resp. infinitely often) among the successor trees $\{T[y]\}$ above $x$ if there are only finitely many (resp. infinitely many) immediate successors $y$ of $x$ such that $T[y] \cong I$. In general, when we speak of an isomorphism type $I$ occurring in a tree $T$, we mean that there is a node $a \in T$ such that $T[a] \cong I$.

The domain of $T$ is always assumed to be $\omega$, and we have a computable approximation to $T$ by:

$$
T_{s}=\{0,1, \ldots s-1\} \cup\{r\},
$$

where $r$ is the root of $T$. We restate Lemma 2.10 because it will be used repeatedly.

Lemma 2.10 Suppose $T$ and $T^{\prime}$ are two trees of finite height and strongly finite type. If each of $T$ and $T^{\prime}$ embeds into the other, then they are isomorphic.

Lemma 3.2 Suppose that $T$ is a tree of finite height which is of finite type but not of strongly finite type. Then there exists an $\omega$-branch point $y_{0} \in T$ such that all successor trees above $y_{0}$ are of strongly finite type, and such that some successor tree which appears only finitely often above $y_{0}$ embeds into some successor tree appearing infinitely often above $y_{0}$.

Proof. Let $y_{0}$ be maximal in $T$ among nodes which are not of strongly finite type. (This set is non-empty, since it must include the root of $T$.) Then $y_{0}$ must be $\omega$-branching, and every successor tree above $y_{0}$ is of strongly finite type. The only way $y_{0}$ can fail to be of strongly finite type is for there to be distinct successor trees $T_{j} \hookrightarrow T_{k}$ above $y_{0}$ such that infinitely many other successor trees above $y_{0}$ are isomorphic to $T_{k}$. Since $y_{0}$ is of finite type, however, the isomorphism type of $T_{j}$ must appear only finitely often above $y_{0}$.

Lemma 3.3 Let $\left\{T_{1}, \ldots T_{n}\right\}$ be any collection of trees of weakly finite type. Then there exist finite trees $S_{1}, \ldots S_{n}$ such that for all $i$ and $j$ :

$$
S_{i} \hookrightarrow T_{j} \Longleftrightarrow T_{i} \hookrightarrow T_{j}
$$


Proof. To build $S_{i}$, consider the set $A_{i}=\left\{j \leq n: T_{i} \nrightarrow T_{j}\right\}$. For each $j \in A_{i}$, there is a finite subtree $S_{i, j} \subseteq T_{i}$ such that $S_{i, j} \nrightarrow T_{j}$, by Lemma 2.9. Let $S_{i}$ be the union of all these subtrees, for all $j \in A_{i}$. (If $A_{i}$ is empty, take $S_{i}$ to be a single node.)

We will need a version of Kruskal's Theorem for trees of weakly finite type. In order to prove this theorem, we use labeled finite trees. For our purposes, a labeled finite tree is a finite tree $S$ together with a function $l: S \rightarrow\{0,1, \omega\}$. The elements of the set $\{0,1, \omega\}$ are called labels and the function $l$ is called the labeling function. Let $S_{1}$ and $S_{2}$ be labeled trees with labeling functions $l_{1}$ and $l_{2}$. An embedding $f: S_{1} \hookrightarrow S_{2}$ respects the labels if for every $x \in S_{1}, l_{1}(x) \leq l_{2}(f(x))$. A proof of the following version of Kruskal's Theorem can be found in either [19] or [31]. (In fact, for our purposes, we can assume that there is a uniform finite bound $n$ on the heights of the trees $S_{i}$. This assumption leads to a far simpler proof.)

Theorem 3.4 (Kruskal) Let $\left\{S_{i}: i \in \omega\right\}$ be an infinite collection of finite trees, each with a labeling $l_{i}$. Then there exist $i<j$ in $\omega$ and an embedding $f: S_{i} \hookrightarrow S_{j}$ (preserving the infimum function) such that for every $x \in S_{i}, l_{i}(x) \leq l_{j}(f(x))$.

Lemma 3.5 (Kruskal's Theorem for weakly finite type) Fix $n \in \omega$, and let $\left\{T_{i}: i \in\right.$ $\omega\}$ be an infinite collection of trees of weakly finite type, with $\mathrm{ht}\left(T_{i}\right) \leq n$ for all $i$. Then there exist $i<j$ in $\omega$ such that $T_{i}$ can be embedded in $T_{j}$.

Corollary 3.6 Let $\left\{T_{i}: i \in \omega\right\}$ be an infinite collection of trees of weakly finite type, with ht $\left(T_{i}\right) \leq n$ for all $i$. Then there exists $m \in \omega$ such that for every $i>m, T_{i}$ can be embedded in some $T_{j}$ with $j>i$, and some $T_{k}$ with $k<i$ can be embedded in $T_{i}$.

Proof. By Lemma 3.5 both $\left\{i \in \omega \mid \forall j>i\left(T_{i} \hookrightarrow T_{j}\right)\right\}$ and $\left\{i \in \omega \mid \forall k<i\left(T_{k} \nrightarrow T_{i}\right)\right\}$ must be finite.

Proof of Lemma 3.5. We claim that given the collection $\left\{T_{i}\right\}$, we can build a corresponding collection $\left\{S_{i}\right\}$ of labeled finite trees such that if $i<j$ and there is an embedding of $S_{i}$ into $S_{j}$ which respects the labels, then $T_{i}$ also embeds into $T_{j}$. To prove this claim, we induct on $n$. The case $n=1$ is easy, since there is only one possible tree, containing a single node; we take $S_{i}=T_{i}$ for each $i$, labeling the node of each $S_{i}$ with 1 .

Now assume the claim for $n$. For each tree $T_{i}$ given by the lemma, let $r_{i}$ be the root of $T_{i}$, and let $I_{i, 1}, \ldots I_{i, m_{i}}$ be the (finitely many) distinct isomorphism types of successor trees above $r_{i}$. Then the inductive hypothesis applies to the set

$$
\left\{I_{i, k}: i \in \omega, 1 \leq k \leq m_{i}\right\}
$$

yielding a set $\left\{S_{i, k}\right\}$ of corresponding labeled finite trees. Define $S_{i}$ inductively as follows:

- $S_{i}$ has a root $s_{i}$, labeled with 1 ; 
- $S_{i}$ has a chain $u_{i, 1} \prec u_{i, 2} \prec \cdots \prec u_{i, n}$, each labeled with 0 , and with $u_{i, 1}$ an immediate successor of $s_{i}$

- For each isomorphism type $I_{i, k}$ which appears only finitely often - say $p$ times - among the successor trees above $r_{i}$ in $T_{i}$, add $p$ copies of the corresponding $S_{i, k}$ as successor trees above $s_{i}$ in $S_{i}$, with the root of each of these successor trees labeled with a 1 ; and

- For each isomorphism type $I_{i, k}$ which appears infinitely often among the successor trees above $r_{i}$ in $T_{i}$, add a copy of the corresponding $S_{i, k}$ as a successor tree above $s_{i}$ in $S_{i}$, but labeling its root with $\omega$, rather than 1 .

We have changed the labels on the roots of certain finite successor trees $S_{i, k}$, but only by changing the label of the root from 1 to $\omega$, so we have not introduced any new embeddings among the $S_{i, k}$ 's.

Now if $f$ is an embedding of $S_{i}$ into $S_{j}(j>i)$ which preserves infima and respects labels, then $f$ must map the root $s_{i}$ to $s_{j}$, since both trees have height $n$. (This was the purpose of the chains $\left\{u_{i, k}\right\}$ and $\left\{u_{j, k}\right\}$.) Hence each successor tree in $S_{i}$ maps into a distinct successor tree in $S_{j}$, since $f$ preserves infima. It follows that each isomorphism type among the successor trees in $T_{i}$ maps into some successor tree in $T_{j}$. Because of the labeling with 0,1 and $\omega$ on $S_{i}$, we know that no $S_{i, k}$ maps into the chain above $u_{j, 1}$, and that each infinite-appearing successor tree in $T_{i}$ maps into an infinite-appearing successor tree in $T_{j}$ (or possibly into an infiniteappearing subtree of a finite-appearing successor tree in $T_{j}$ ). Finally, each finite-appearing successor tree in $T_{i}$ appeared just as many times in $S_{i}$, and hence there must be sufficiently many successor trees in $T_{j}$ for each copy of the type to map to.

Applying Theorem 3.4 to our set $\left\{S_{i}\right\}$, we get an $f$ with precisely the properties required by the claim. Hence some $T_{i}$ embeds into some $T_{j}$ with $j>i$.

We will be interested in the minimal elements (under embedding) of various sets of trees of weakly finite type. Let $\mathcal{T}$ be a set of trees of weakly finite type for which there is a finite bound on the height of the trees appearing in $\mathcal{T}$. Lemma 3.5 says that $\mathcal{T}$ together with the embeddability relation forms a well-quasi-order. Therefore, $\mathcal{T}$ satisfies both the descending chain condition that any strictly descending chain in $\mathcal{T}$ under embeddability is finite and the incomparable chain condition that any anti-chain under the embeddability relation is finite. (See [19] for more details on these properties. We are using the fact that a quasi-order is a well-quasi-order if and only if it satisfies both of these conditions.) In this context, the appropriate definition of "minimal" is based on equivalence classes under $\equiv$ rather than under $\cong$.

Definition 3.7 $T \in \mathcal{T}$ is minimal in $\mathcal{T}$ if for every $T^{\prime} \in \mathcal{T}$ such that $T^{\prime} \hookrightarrow T$, we have $T \equiv T^{\prime}$.

For trees of strongly finite type, this is equivalent to the standard definition of minimal under $\cong$, by Lemma 2.10. However, trees of weakly finite type do not necessarily satisfy 
this lemma; they form only a quasi-order under $\hookrightarrow$, not a partial order. (The notion of a quasi-order plays no explicit role in the paper after the next corollary.)

Corollary 3.8 Let $\mathcal{T}$ be an infinite collection of trees of weakly finite type, with $\mathrm{ht}(T) \leq n$ for all $T \in \mathcal{T}$. Then there exists a finite set $\mathcal{M} \subseteq \mathcal{T}$ of minimal elements of $\mathcal{T}$ (under the embedding relation) such that for every $T \in \mathcal{T}$ there exists $T^{\prime} \in \mathcal{M}$ with $T^{\prime} \hookrightarrow T$.

Proof. Let $\mathcal{S}$ be the set of all minimal elements of $\mathcal{T}$, and let $\mathcal{M}$ contain exactly one representative from each $\equiv$-equivalence class in $\mathcal{S}$. Then the incomparable chain condition implies that $\mathcal{M}$ must be finite, and the descending chain condition implies that every $T \in \mathcal{T}$ contains a subtree from $\mathcal{M}$.

This corollary will frequently be applied with $\mathcal{T}$ being either the set $\left\{T: T \hookrightarrow T_{0}\right\}$ (for some fixed $\left.T_{0}\right)$ or the set $\left\{T: T_{0} \hookrightarrow T \& T_{0} \not T\right\}$. We will need one last general fact about embeddings between finite height trees of finite type.

Lemma 3.9 Let $T_{0}$ and $T_{1}$ be finite height trees of finite type and let $\mathcal{I}_{i}$ be the finite set of isomorphism types of successor trees which occur infinitely often immediately above the root in $T_{i}$. If $T_{0} \equiv T_{1}$, then $\mathcal{I}_{0}=\mathcal{I}_{1}$.

Proof. We will write $T_{i}[x] \in \mathcal{I}_{i}$ to indicate that the tree $T_{i}[x]$ is a successor tree of the root in $T_{i}$ and that its isomorphism type occurs in $\mathcal{I}_{i}$.

Consider any $T_{0}[x] \in \mathcal{I}_{0}$ and suppose that $T_{0}[x]$ embeds in some $T_{1}[y] \in \mathcal{I}_{1}$. Further, suppose that $T_{1}[y]$ embeds in some $T_{0}[z] \in \mathcal{I}_{0}$. Composing these two embeddings gives that $T_{0}[x] \hookrightarrow T_{0}[z]$. But, because $T_{0}$ has finite type and both $T_{0}[x]$ and $T_{0}[z]$ occur infinitely often, it must be that $T_{0}[x] \cong T_{0}[z]$. Furthermore, $T_{0}[x], T_{1}[y]$ and $T_{0}[z]$ all have strongly finite type since they occur infinitely often. The two embeddings $T_{0}[x] \hookrightarrow T_{1}[y] \hookrightarrow T_{0}[z] \cong T_{0}[x]$ show that $T_{0}[x] \cong T_{1}[y]$ by Lemma 2.10 .

The argument in the previous paragraph establishes the lemma except in the case when there is a type $I \in \mathcal{I}_{i}$ which does not embed in any type $J \in \mathcal{I}_{1-i}$. Without loss of generality, assume that there is a type $I \in \mathcal{I}_{0}$ which does not embed in any type in $\mathcal{I}_{1}$. In this case we will derive a contradiction to the fact that $T_{0}$ and $T_{1}$ have finite height.

For any $c \in T_{j}$, we say that $c$ occurs in the finite part of $T_{j}$ if $c \in T_{j}[a]$ for some $a$ at level 1 of $T_{j}$ for which $T_{j}[a]$ is one of the finitely occurring isomorphism types at level 1 . Otherwise, we say that $c$ occurs in the infinite part of $T_{j}$.

We define a notion of rank for our fixed isomorphism type $I$. A node $a \in T_{j}$ has rank $\operatorname{rk}(a) \geq 0$ if $I$ embeds in $T_{j}[a]$. A node $a$ has $\operatorname{rank} \operatorname{rk}(a) \geq n+1$ if there are infinitely many nodes $c$ for which $a \prec c$ and $\operatorname{rk}(c) \geq n$. There are a number of simple facts that follow from this definition.

Fact 1. If $\operatorname{rk}(a) \geq n$, then $\operatorname{ht}\left(T_{j}[a]\right) \geq \operatorname{ht}(I)+n$. This fact follows by an induction on $n$.

Fact 2. If $c$ lies above the root in $T_{1}$ and $\operatorname{rk}(c) \geq 0$, then $c$ is in the finite part of $T_{1}$. This fact follows since $I \hookrightarrow T_{1}[c]$ and $\mathcal{I}_{1}$ contains no isomorphism types into which $I$ embeds. 
Fact 3. If $c$ lies above the root in $T_{0}$ and $\operatorname{rk}(c) \geq 1$, then $c$ occurs in the finite part of $T_{0}$. For a contradiction, assume that $c$ occurs in the infinite part of $T_{0}$. Then $c$ must occur in some successor tree $T_{0}[a]$ of the root whose isomorphism type is in $\mathcal{I}_{0}$. However, by Fact $1, \operatorname{ht}\left(T_{0}[a]\right)>\operatorname{ht}(I)$, so $T_{0}[a] \not I$. This means $I \hookrightarrow T_{0}[a]$ is an embedding relation between distinct infinitely occurring isomorphism types at level 1 of $T_{0}$, which contradicts the fact that $T_{0}$ has finite type.

Fact 4. For any $k, n \geq 1$, if $a_{1}, \ldots, a_{k} \in T_{j}$ satisfy $\operatorname{rk}\left(a_{i}\right) \geq n$, then there are $b_{1}, \ldots, b_{k} \in$ $T_{1-j}$ which satisfy $\mathrm{rk}\left(b_{i}\right) \geq n$. To establish this fact, fix any embedding $f: T_{j} \hookrightarrow T_{1-j}$ and let $b_{i}=f\left(a_{i}\right)$. The result follows by induction on $n$.

By Fact 1, to derive a contradiction with the fact that $T_{1}$ has finite height, it suffices to show that for each $n \geq 1, T_{1}$ must have a node $a$ with $\operatorname{rk}(a) \geq n$. We establish this by induction on $n$. Fix embeddings $f: T_{0} \hookrightarrow T_{1}$ and $g: T_{1} \hookrightarrow T_{0}$.

For the case of $n=1$, we claim that the fact that $I$ does not embed into any element of $\mathcal{I}_{1}$ implies that there is an $a \in T_{1}$ for which $\operatorname{rk}(a) \geq 1$. To prove this claim, consider the embedding $f: T_{0} \hookrightarrow T_{1}$. Each copy $T_{0}[d]$ of $I$ in the infinite part of $T_{0}$ must map into the finite part of $T_{1}$ under $f$. Therefore, there must be a node $a$ at level 1 of $T_{1}$ (in the finite part) for which infinitely many copies of $I$ embed into $T_{1}[a]$. For this $a, \operatorname{rk}(a) \geq 1$.

Assume by induction that the fact that $I$ does not embed into any element of $\mathcal{I}_{1}$ implies that there is an $a \in T_{1}$ with $\operatorname{rk}(a) \geq n$.

Fix $a_{1} \in T_{1}$ such that $\operatorname{rk}\left(a_{1}\right) \geq n$. We claim that there is an element $a_{2} \in T_{1}$ with $a_{2} \neq a_{1}$ and $\operatorname{rk}\left(a_{2}\right) \geq n$. To prove this claim, notice that by Fact 4 , we know that $g\left(a_{1}\right)=b_{1} \in T_{0}$ satisfies $\operatorname{rk}\left(b_{1}\right) \geq n$. Furthermore, setting $c_{1}=f\left(b_{1}\right)$, we have that $\operatorname{rk}\left(c_{1}\right) \geq n$.

We split into two cases depending on whether $a_{1}=c_{1}$ or $a_{1} \neq c_{1}$. If $a_{1} \neq c_{1}$, then we let $a_{2}=c_{1}$ and we are done with the claim. Otherwise, if $a_{1}=c_{1}$, then $f\left(b_{1}\right)=a_{1}$ and $g\left(a_{1}\right)=b_{1}$. Therefore $f$ maps $T_{0}\left[b_{1}\right]$ into $T_{1}\left[a_{1}\right]$ and $g$ maps $T_{1}\left[a_{1}\right]$ into $T_{0}\left[b_{1}\right]$, and moreover, restricting $f$ to $T_{0} \backslash T_{0}\left[b_{1}\right]$ and restricting $g$ to $T_{1} \backslash T_{1}\left[a_{1}\right]$ shows that $\left(T_{0} \backslash T_{0}\left[b_{1}\right]\right) \equiv\left(T_{1} \backslash T_{1}\left[a_{1}\right]\right)$. By the induction hypothesis (which applies since we have only removed a portion of the finite parts of $T_{0}$ and $T_{1}$ by Facts 2 and 3 and therefore not changed the infinite part of either tree), there is an element $a_{2} \in T_{1} \backslash T_{1}\left[a_{1}\right]$ for which $\operatorname{rk}\left(a_{2}\right) \geq n$. This establishes the claim.

More generally, for any $k \geq 1$, if $a_{1}, \ldots, a_{k} \in T_{1}$ have $\operatorname{rk}\left(a_{i}\right) \geq n$, then we can fix $g\left(a_{1}\right)=b_{1}, \ldots, g\left(a_{k}\right)=b_{k} \in T_{0}$ with $\operatorname{rk}\left(b_{i}\right) \geq n$. Setting $c_{i}=f\left(b_{i}\right)$ and splitting into cases as above, we get the existence of $a_{k+1} \in T_{1}$ with $\operatorname{rk}\left(a_{k+1}\right) \geq n$. Therefore, there must be infinitely many nodes in the finite part of $T_{1}$ which have rank $\geq n$. We can fix a node $a$ at level 1 of $T_{1}$ (and in the finite part) for which infinitely many of these nodes occur in $T_{1}[a]$. Then, $\operatorname{rk}(a) \geq n+1$ as required.

We now begin the constructions which will ultimately prove Proposition 3.1. Suppose $T$ has finite height, but is not of finite type. In this section we consider the case when the root $r$ is the only node of $T$ which fails to be of finite type. Then $T$ must be $\omega$-branching at $r$, and we write $x_{0}, x_{1}, \ldots$ for the immediate successors of $r$ in $T$. We present several constructions concerning various ways in which $T$ could fail to have finite type and we prove in each case that $T$ is not computably categorical. After these constructions, we prove Proposition 3.1 by 
showing that we have considered all possible cases. We present the first proof in the most detail since many of the later arguments will have similar features.

Lemma 3.10 Let $T$ be a tree of finite height with root $r$, and suppose that each node above $r$ in $T$ is of finite type. Suppose there is an isomorphism type $I_{0}$ which is not of strongly finite type and appears infinitely often as a successor tree above $r$. If only finitely many other isomorphism types $I^{\prime}$ appearing above $r$ satisfy $I^{\prime} \hookrightarrow I_{0}$, then $T$ is not computably categorical.

Proof. First, we establish that there is a $\Delta_{2}^{0}$ procedure which identifies the immediate successors $x$ of $r$ such that $T[x] \equiv I_{0}$. Let $\mathcal{F}$ be the set of all isomorphism types $I$ appearing as successor trees above $r$ such that $I \nrightarrow I_{0}$. Let $\left\{I_{1}, \ldots I_{m}\right\}$ be a set of minimal elements (under $\hookrightarrow$ ) of $\mathcal{F}$ as given by Corollary 3.8, so that every $I \in \mathcal{F}$ is a supertree of some $I_{i}$. By Lemma 3.3, each $I_{i}(i>0)$ contains a finite subtree $S_{i}$ such that $S_{i} \hookrightarrow I_{0}$. Therefore, a node $x$ at level 1 in $T$ satisfies $T[x] \hookrightarrow I_{0}$ if and only if $\forall s \forall i \leq m\left(S_{i} \hookrightarrow T_{s}[x]\right)$.

Consider the finite number of isomorphism types $I^{\prime} \hookrightarrow I_{0}$. For each such $I^{\prime}$ for which $I_{0} \leftrightarrow I^{\prime}$, there is a finite subtree $Q^{\prime}$ of $I_{0}$ such that $Q^{\prime} \nrightarrow I^{\prime}$. Taking the union of these finite trees $Q^{\prime}$ gives a finite tree $Q$ such that $Q \hookrightarrow I_{0}$ and for all $I^{\prime}$ as above, $Q \hookrightarrow I^{\prime}$. Therefore, an immediate successor $x$ of $r$ satisfies $T[x] \equiv I_{0}$ if and only if

$$
\forall s \forall i \leq m\left(S_{i} \hookrightarrow T_{s}[x]\right) \wedge \exists s\left(Q \hookrightarrow T_{s}[x]\right) .
$$

Since we can clearly identify the immediate successors of $r$ in a $\Delta_{2}^{0}$ manner, this definition shows that we can identify the immediate successors $x$ of $r$ which satisfy $T[x] \equiv I_{0}$ with a $\Delta_{2}^{0}$ procedure.

During the construction, we try to identify trees $T[x]$ for which $x$ is an immediate successor of $r$ and $T[x] \equiv I_{0}$. We say that we believe $T[x] \equiv I_{0}$ at stage $s$ if $x$ is an immediate successor of $r$ in $T_{s}, Q \hookrightarrow T_{s}[x]$ and $S_{i} \hookrightarrow T_{s}[x]$ for all $i \leq m$. Without loss of generality, we assume that the finite tree $Q$ has the same height as $I_{0}$. Therefore, if we believe $T[x] \equiv I_{0}$ at stage $s$, then any embedding of $T_{s}[x]$ into $I_{0}$ must send $x$ to the root node of $I_{0}$.

Since it is of finite type and not of strongly finite type, $I_{0}$ must contain an $\omega$-branching node $y_{0}$ satisfying Lemma 3.2. Fix such a node $y_{0}$ and let $J$ denote the isomorphism type of $I_{0}\left[y_{0}\right]$. By Lemma 3.2, there exist successor isomorphism types $J_{0} \hookrightarrow J_{1}$ of $J$ with $J_{0}$ occurring only finitely often above $y_{0}$ and $J_{1}$ occurring infinitely often above $y_{0}$. Moreover, Lemma 3.2 ensures that both $J_{0}$ and $J_{1}$ are of strongly finite type, so Lemma 2.10 guarantees that $J_{1} \nrightarrow J_{0}$.

We will build a tree $T^{\prime}$ and an embedding $f: T \rightarrow T^{\prime}$ such that $T^{\prime}$ is equal to the range of $f$ together with infinitely many copies of $I_{0}$ which are attached to the root of $T^{\prime}$. Our strategy to diagonalize against $\varphi_{e}: T \rightarrow T^{\prime}$ being an isomorphism will roughly be to identify subtrees $T[z]$ which satisfy $T[z] \equiv J_{0}$. Once we find such a subtree, we make sure that $J_{1}$ embeds into our subtree $T^{\prime}\left[\varphi_{e}(z)\right]$. Because $J_{1} \nrightarrow J_{0}$, it cannot be that $T^{\prime}\left[\varphi_{e}(z)\right] \cong T[z]$. (In fact, it cannot even be that $T^{\prime}\left[\varphi_{e}(z)\right] \hookrightarrow T[z]$.)

Consider the finite number of isomorphism types $I$ which occur as successor trees in $T$ and which satisfy $I \equiv I_{0}$. By Lemma 2.6, each such $I$ contains only finitely many isomorphism 
types of the form $I[a]$ for $a \in I$. Therefore, we can list the types of all such $I[a]$ where $I$ is as above and $a \in I$ as $K_{1}, \ldots, K_{n}$. (This list includes the types of trees $I[a]$ of any level, not just the types of the successor trees of the root of $I$.) As above, we can use finite trees to distinguish these types up to $\equiv$-equivalence. That is, for $K_{1}$ there are finitely many types $K_{i} \nrightarrow K_{1}$. List these trees as $K_{i_{1}}, \ldots, K_{i_{l}}$. For each $k \leq l$, there is a finite subtree $S_{i_{k}}^{\prime}$ of $K_{i_{k}}$ such that $S_{i_{k}}^{\prime} \nrightarrow K_{1}$. Similarly, there are finitely many $K_{j}$ such that $K_{1} \nrightarrow K_{j}$. For each such $K_{j}$, there is a finite subtree $S_{j}^{\prime \prime}$ of $K_{1}$ such that $S_{j}^{\prime \prime} \nrightarrow K_{j}$. Taking the union of these trees yields a finite subtree $S^{\prime \prime}$ of $K_{1}$ such that $S^{\prime \prime} \nrightarrow K_{j}$ for any such $j$. Therefore, if $T[x] \equiv I_{0}$ and $x \prec u$, then $T[u] \equiv K_{1}$ if and only if

$$
\forall s \forall k \leq l\left(S_{i_{k}}^{\prime} \hookrightarrow T_{s}[u]\right) \wedge \exists s\left(S^{\prime \prime} \hookrightarrow T_{s}[u]\right) .
$$

At stage $s$, we believe that $T_{s}[u] \equiv K_{1}$ if and only if the following conditions are satisfied: there is a node $x \prec u$ in $T_{s}$ such that we believe $T_{s}[x] \equiv I_{0} ; S^{\prime \prime} \hookrightarrow T_{s}[u]$; and for all $k \leq l$, $S_{i_{k}}^{\prime} \nrightarrow T_{s}[u]$.

We can perform similar calculations for the other types $K_{i}$. During the construction, if $x$ is an immediate successor of $r$ with $x \prec u$ for some $u$ and we believe $T[x] \equiv I_{0}$, then we can determine using finite trees which $K_{j}$ we believe satisfies $T[u] \equiv K_{j}$ (if any). From the nature of the conditions, it is clear that if we believe infinitely often that $T[x] \equiv I_{0}$ and $T[u] \equiv K_{j}$, then in fact these equivalences hold.

Notice that the isomorphism types $J, J_{0}$, and $J_{1}$ occur among the types $K_{1}, \ldots, K_{n}$. Furthermore, by Lemma 3.9, for any node $y$ such that $T[y] \equiv J, T[y]$ must have infinitely many successor trees which are isomorphic to $J_{1}$.

We begin to describe the construction. Assume that $T$ is approximated in finite stages by $T_{s}$. Without loss of generality, we assume that we know the root of $T$. We build $T_{s}^{\prime}$ and a sequence of embeddings $f_{s}: T_{s} \rightarrow T_{s}^{\prime}$. $T_{s}^{\prime}$ will consist of the range of $f_{s}$ (which is a finite tree isomorphic to $T_{s}$ by $f_{s}$ ) together with finitely many subtrees which are isomorphic to $I_{0}$ and which are attached to the root of $T_{s}^{\prime}$.

We say that a tuple $\langle x, y, z\rangle$ is special in $T$ if $x$ is an immediate successor of the root, $T[x] \equiv I_{0}, x \prec y, T[y] \equiv J, z$ is an immediate successor of $y$, and $T[z] \equiv J_{0}$. We say that $\langle x, y, z\rangle$ is special at stage $s+1$ if $x, y, z \in T_{s}$ and we believe all of these conditions at stage $s$. Because we have $\Delta_{2}^{0}$ approximations to these conditions, we know that $\langle x, y, z\rangle$ is special in $T$ if and only if there is a stage $s$ such that for all $t \geq s,\langle x, y, z\rangle$ is special at stage $t$.

Recall that we have requirements $\mathcal{R}_{e}$, which attempts to diagonalize against $\varphi_{e}$ being an isomorphism, and $\mathcal{N}_{u}$, which attempts to make the limit of $f_{s}(u)$ defined so that $f$ is $\Delta_{2}^{0}$. The basic strategy for $\mathcal{R}_{e}$ is to define a witness tuple $\langle x, y, z\rangle$ which we believe is special and wait for $\varphi_{e}(x)=x^{\prime}, \varphi_{e}(y)=y^{\prime}$, and $\varphi_{e}(z)=z^{\prime}$ to converge. We next want to determine if we believe that $\left\langle x^{\prime}, y^{\prime}, z^{\prime}\right\rangle$ is going to be special in $T^{\prime}$. This amounts to letting $f_{s}^{-1}\left(x^{\prime}\right)=a$, $f_{s}^{-1}\left(y^{\prime}\right)=b$ and $f_{s}^{-1}\left(z^{\prime}\right)=c$, and asking whether we currently believe $\langle a, b, c\rangle$ is special in $T$. If the answer is no, then it appears that $\mathcal{R}_{e}$ is not an isomorphism and we do not need to diagonalize. If the answer is yes, then we want to actively diagonalize.

Assume that not only do we believe that $\langle x, y, z\rangle$ and $\langle a, b, c\rangle$ are special in $T$ at stage $s$ of the construction, but they really are special in $T$. Then, there is an embedding of $T_{s}[a] \hookrightarrow I_{0}$ 
which maps $c$ to the base of a tree of type $J_{1}$. (We prove the existence of such an embedding below when we do the formal construction.) Therefore, we can diagonalize by using the embedding of $T_{s}[a]$ into $I_{0}$ to turn $T_{s}^{\prime}\left[x^{\prime}\right]$ into a copy of $I_{0}$ for which $T_{s}^{\prime}\left[z^{\prime}\right]$ becomes a tree into which $J_{1}$ embeds. We now know $J_{1} \hookrightarrow T^{\prime}\left[z^{\prime}\right]$ and $T[z] \hookrightarrow J_{0}$. Therefore, if $\varphi_{e}$ is an isomorphism, then $T^{\prime}\left[z^{\prime}\right] \hookrightarrow T[z]$ and hence $J_{1} \hookrightarrow J_{0}$, which contradicts our choice of $J_{0}$.

We also need to redefine $f_{s+1}$ on $T_{s}[a]$ since we have turned $T_{s}^{\prime}\left[x^{\prime}\right]$ into $I_{0}$ and we do not know that $T_{s}[a]$ has isomorphism type $I_{0}$. Therefore, we add new elements to $T_{s+1}^{\prime}$ and define the map $f_{s+1}$ to send the tree $T_{s}[a]$ to these new elements. Notice that once an element $y^{\prime} \in T^{\prime}$ has left the range of $f$, it will never return to the range of $f$. Therefore, we have that for all $y^{\prime} \in T^{\prime}$, either $f_{s}^{-1}\left(y^{\prime}\right)$ converges or $y^{\prime}$ is permanently part of an auxiliary copy of $I_{0}$. Hence we do not need to explicitly discuss the requirements $\mathcal{M}_{u}$ in this construction.

This action of changing the map so that $f_{s}(a) \neq f_{s+1}(a)$ conflicts with the requirements of the form $\mathcal{N}_{u}$ for $u \in T_{s}[a]$. To fix this problem, we give $\mathcal{R}_{e} e+1$ many witness tuples with distinct first components and we do not allow $\mathcal{R}_{e}$ to use the tuple $\langle x, y, z\rangle$ to diagonalize if $f_{s}^{-1}\left(\varphi_{e}(x)\right) \prec u$ for any $u \leq e$. That is, we give $\mathcal{N}_{u}$ higher priority than $\mathcal{R}_{e}$ if $u<e$. Since each $\mathcal{N}_{u}$ can stop $\mathcal{R}_{e}$ from using at most one node at level one with which to diagonalize, assigning $e+1$ tuples to $\mathcal{R}_{e}$ is enough to guarantee that either $\mathcal{R}_{e}$ will be allowed to diagonalize with one of these tuples or $\mathcal{R}_{e}$ will be satisfied in a trivial way, such as $\varphi_{e}$ not being one-to-one or not respecting the ordering.

There is a second possible worry for the basic strategy for $\mathcal{R}_{e}$. Assume that $\langle x, y, z\rangle$ is a witness tuple for $\mathcal{R}_{e}$ and $\left\langle x^{\prime}, y^{\prime}, z^{\prime}\right\rangle$ is as above. Since $T_{s}^{\prime}$ consists of the range of $f_{s}$ together with additional copies of $I_{0}$, it is possible that the elements of $\left\langle x^{\prime}, y^{\prime}, z^{\prime}\right\rangle$ sit in one of the copies of $I_{0}$. In this case, it makes no sense to look at $f_{s}^{-1}$ on the values $x^{\prime}, y^{\prime}$ and $z^{\prime}$ since these elements are not in the range of $f_{s}$. Of course, if it is not the case that $x^{\prime} \prec y^{\prime} \prec z^{\prime}$ in $T_{s}^{\prime}$, then we have beaten $\varphi_{e}$ trivially. Otherwise, $\mathcal{R}_{e}$ can check if $x^{\prime}$ is the root of the copy of $I_{0}, y^{\prime}$ is the root of a tree which is $\equiv J$ and $z^{\prime}$ is the root of a tree which is $\equiv J_{0}$. (We will assume that when we add a copy of $I_{0}$ to $T^{\prime}$, we add a "nice" copy in which we know the isomorphism type of each subtree of the form $T^{\prime}[a]$ for $a$ in this copy of $I_{0}$. This is possible since $I_{0}$ contains only finitely many such isomorphism types.) If not, then $\mathcal{R}_{e}$ has already won. If so, then $\mathcal{R}_{e}$ can win by turning $T^{\prime}\left[z^{\prime}\right]$ into a copy of $J_{1}$ (which is possible because $J_{0} \hookrightarrow J_{1}$ ) and adding a new copy of the old $T^{\prime}\left[z^{\prime}\right]$ above $T^{\prime}\left[y^{\prime}\right]$. Because $T^{\prime}\left[y^{\prime}\right]$ bounds infinitely many copies of $J_{1}$, adding an extra copy does not change its isomorphism type. So, we still have $T^{\prime}\left[y^{\prime}\right] \equiv J$ and $T^{\prime}\left[x^{\prime}\right] \cong I_{0}$. This action wins $\mathcal{R}_{e}$ as above, since $J_{1} \hookrightarrow T^{\prime}\left[z^{\prime}\right]$ and $T[z] \hookrightarrow J_{0}$.

However, notice that if we performed this action infinitely often with the same copy of $I_{0}$, then we might move the same subtree of this copy of $I_{0}$ infinitely often and not have a copy of $I_{0}$ in the limit. Therefore, we need to restrict this action from happening infinitely often. When a requirement $\mathcal{R}_{e}$ creates a copy of $I_{0}$, it marks it with the same priority as $\mathcal{R}_{e}$. We only allow requirements $\mathcal{R}_{i}$ with $i<e$ to diagonalize using this copy of $I_{0}$ as described above. This causes only finitely additions to the tree after it is defined, so it really does have type $I_{0}$ in the limit.

Because $\mathcal{R}_{e}$ is not allowed to use a witness tuple $\langle x, y, z\rangle$ when $\varphi_{e}(x)$ converges to the root 
of a copy of $I_{0}$ which is marked by a requirement of higher priority, we have to allow $\mathcal{R}_{e}$ extra witness tuples. Each time $\mathcal{R}_{i}$ with $i<e$ marks a new copy of $I_{0}, \mathcal{R}_{e}$ is given an extra witness tuple. This action will only occur finitely often, so in the end, $\mathcal{R}_{e}$ has $e+n+1$ many witness tuples, where $n$ is the number of copies of $I_{0}$ marked by requirements of higher priority. We cannot fix the number $n$ at the beginning of the construction since finitely often a requirement of higher priority may create a copy of $I_{0}$ thinking that it is diagonalizing against a particular witness tuple only to discover later that this witness tuple was not actually special.

We turn to a more formal description of the construction. At each stage $s$, we define a finite list of tuples which are special at that stage and which have distinct first components. More formally, let $\left\langle x_{0}, y_{0}, z_{0}\right\rangle$ be the $\leq_{\mathbb{N}}$ least tuple (under a fixed coding of $\mathbb{N}^{3}$ ) that is special at stage $s$. Let $\left\langle x_{i+1}, y_{i+1}, z_{i+1}\right\rangle$ be the $\leq_{\mathbb{N}^{-}}$least tuple greater than $\left\langle x_{i}, y_{i}, z_{i}\right\rangle$ which is special at stage $s$ and such that $x_{i+1}$ is not equal to $x_{j}$ for any $j \leq i$. List these tuples at stage $s$ by

$$
\left\langle x_{0, s}, y_{0, s}, z_{0, s}\right\rangle, \ldots,\left\langle x_{p_{s}, s}, y_{p_{s}, s}, z_{p_{s}, s}\right\rangle \text {. }
$$

At stage $s$, we assign $e+n+1$ many of these tuples to the requirement $\mathcal{R}_{e}$, where $n$ is the number of copies of $I_{0}$ created by requirements of higher priority by stage $s$. If there are not enough tuples for $\mathcal{R}_{e}$ to get its full set of tuples, then it is not assigned any tuples. $\mathcal{R}_{e}$ may be later declared to be satisfied by one of the tuples it has been assigned. If that tuple ever changes, then $\mathcal{R}_{e}$ is said to be injured and it is no longer satisfied.

Because there are infinitely many copies of $I_{0}$ attached to the root of $T$, there is an infinite set of special tuples in $T$ which have pairwise distinct first components. Therefore, each tuple of the form $\left\langle x_{m, s}, y_{m, s}, z_{m, s}\right\rangle$ is eventually defined and reaches a limit $\left\langle x_{m}, y_{m}, z_{m}\right\rangle$, which is a special tuple in $T$. So, each requirement $\mathcal{R}_{e}$ is eventually assigned a complete set of special tuples.

At stage $s+1$ we extend the isomorphism $f_{s}$ to $f_{s+1}: T_{s+1} \rightarrow T_{s+1}^{\prime}$ by adding fresh elements to $T_{s+1}^{\prime}$, unless there exists an $\mathcal{R}_{e}$ requirement with $e \leq s$ which requires attention. If some $\mathcal{R}_{e}$ requires attention, we let the highest priority such requirement act. Below we define when $\mathcal{R}_{e}$ requires attention and what action $\mathcal{R}_{e}$ takes when it is allowed to act.

Assume that $\mathcal{R}_{e}$ is assigned the tuple $\langle x, y, z\rangle$ during the construction. $\mathcal{R}_{e}$ waits for $\varphi_{e}(x)$, $\varphi_{e}(y)$ and $\varphi_{e}(z)$ to converge to some $x^{\prime}, y^{\prime}$, and $z^{\prime}$ respectively. Once these computations converge (say at stage $s+1$ ), $\mathcal{R}_{e}$ checks for two possible easy wins. First, if any of the elements is not in $T_{s}^{\prime}$, then $\mathcal{R}_{e}$ wins by making sure that these elements are all placed in $T_{s+1}^{\prime}$ and they do not satisfy $x^{\prime} \prec y^{\prime} \prec z^{\prime}$. Second, if either $\varphi_{e}$ is not one-to-one or $x^{\prime}$ is equal to the root in $T^{\prime}$ or it is not the case that $x^{\prime} \prec y^{\prime} \prec z^{\prime}$, then $\varphi_{e}$ cannot be an isomorphism and $\mathcal{R}_{e}$ wins without performing any action.

Assume that $\mathcal{R}_{e}$ does not win trivially and that it has not been declared satisfied by one of its tuples. The action for $\mathcal{R}_{e}$ splits into two cases. Either $x^{\prime}, y^{\prime}$ and $z^{\prime}$ are all in the range of $f_{s}$ or else one of these elements falls outside the range of $f_{s}$. We first consider the case when all the elements are in the range of $f_{s}$. In this case, $\mathcal{R}_{e}$ checks if $\left\langle f_{s}^{-1}\left(x^{\prime}\right), f_{s}^{-1}\left(y^{\prime}\right), f_{s}^{-1}\left(z^{\prime}\right)\right\rangle$ is special. If not, then $\mathcal{R}_{e}$ does not require attention. If this tuple is special, then $\mathcal{R}_{e}$ checks if there is an element $u \in T$ with $u<e$ and $f_{s}^{-1}\left(x^{\prime}\right) \prec u$. If so, then the requirement $\mathcal{N}_{u}$ takes precedence over $\mathcal{R}_{e}$ and prevents $\mathcal{R}_{e}$ from acting. Otherwise, $\mathcal{R}_{e}$ requires attention. 
When $\mathcal{R}_{e}$ is allowed to act, it begins a concurrent search for one of the following:

1. an embedding $T_{s}^{\prime}\left[x^{\prime}\right] \hookrightarrow I_{0}$ which sends $x^{\prime}$ to the root of $I_{0}$ and sends $z^{\prime}$ to the base of a tree into which the isomorphism type $J_{1}$ embeds; or

2. a stage $t \geq s$ at which we no longer believe $\left\langle f_{s}^{-1}\left(x^{\prime}\right), f_{s}^{-1}\left(y^{\prime}\right), f_{s}^{-1}\left(z^{\prime}\right)\right\rangle$ is special.

One comment is necessary to explain condition (1). Because $I_{0}$ has finite type and is computable, we can assume that we have a nice copy of $I_{0}$ in which we know the isomorphism type of each subtree $I_{0}[a]$. Therefore, we know which subtrees $J_{1}$ embeds into, so we can search for an embedding as in (1) in an effective manner. In the sublemmas verifying this construction, we show that this search procedure must terminate.

If we see (2) happen first, then we no longer think that $\mathcal{R}_{e}$ requires attention with this tuple. We extend our tree to $T_{s+1}^{\prime}$ as if no requirement had required attention and go to the next stage. If we see (1) happen first, then we perform the following actions:

- turn $T_{s}^{\prime}\left[x^{\prime}\right]$ into a copy of $I_{0}$ with $x^{\prime}$ as the root and $z^{\prime}$ as the root of a tree into which $J_{1}$ embeds; and

- add extra elements to $T^{\prime}$ to be the new images of the elements above $f_{s}^{-1}\left(x^{\prime}\right)$ in $T$ under $f_{s+1}$; and

- leave $f_{s+1}=f_{s}$ on all other elements from $T$; and

- add new elements to $T^{\prime}$ to correspond to the elements in $T_{s+1} \backslash T_{s}$ and define $f_{s+1}$ in the obvious way;

- declare $\mathcal{R}_{e}$ satisfied with this tuple.

In this case, we say that $\mathcal{R}_{e}$ acts at this stage. Notice that the elements in the new copy of $I_{0}$ in $T^{\prime}$ are outside of the range of $f_{s+1}$. Also, as in the comments explaining the search in (1) above, we know exactly how this copy of $I_{0}$ is constructed in the sense that we know the isomorphism type of each subtree. Furthermore, if $\mathcal{R}_{e}$ is never injured after this stage, then $\langle x, y, z\rangle$ is one of the final tuples assigned to $\mathcal{R}_{e}$. In this case, $T[z] \equiv J_{0}$ but $J_{1} \hookrightarrow T^{\prime}\left[z^{\prime}\right]$. Since $\varphi_{e}(z)=z^{\prime}$ and $J_{1} \nrightarrow J_{0}, \varphi_{e}$ cannot be an isomorphism.

We still need to see what action to take if one of the elements $x^{\prime}, y^{\prime}$ or $z^{\prime}$ is not in the range of $f_{s}$. Because we did not get an easy win for $\mathcal{R}_{e}$, it must be the case that $x^{\prime} \prec y^{\prime} \prec z^{\prime}$ all sit in some successor tree of the root in $T_{s}^{\prime}$. Since one of these elements is not in the range of $f_{s}$, this successor tree must be one of the trees of type $I_{0}$ added to $T_{s}^{\prime}$. If this copy of $I_{0}$ was created by a requirement of higher priority than $\mathcal{R}_{e}$, then $\mathcal{R}_{e}$ does not act at this stage and it ignores this particular witness tuple in future calculations (since it already knows that it is not allowed to diagonalize with this tuple). Otherwise, because we know how such a copy of $I_{0}$ was constructed, we can check whether $x^{\prime}$ is the root of this copy of $I_{0}$, whether $y^{\prime}$ has infinitely many successor trees of type $J_{1}$, whether $z^{\prime}$ is an immediate successor of $y^{\prime}$, and whether $z^{\prime}$ is the base of a subtree which is $\equiv J_{0}$. If any of these conditions fail, then we 
say that $\mathcal{R}_{e}$ is satisfied by this tuple since $\varphi_{e}$ does not appear to be an isomorphism. If all of these conditions hold, then we add a new subtree above $y^{\prime}$ of the same type as the subtree above $z^{\prime}$ and we add elements to the subtree above $z^{\prime}$ to turn it into a copy of $J_{1}$. (In this case, we say that $\mathcal{R}_{e}$ acts at this stage.) Because $y^{\prime}$ must bound infinitely many copies of $J_{1}$, we still have a tree of type $I_{0}$ and now we have diagonalized against $\varphi_{e}$ being an isomorphism. We declare $\mathcal{R}_{e}$ satisfied by this tuple.

This completes the description of the construction. We verify that it succeeds in the following sublemmas.

Sublemma 3.11 In the case when $x^{\prime}, y^{\prime}$ and $z^{\prime}$ are in the range of $f_{s}$, the concurrent search procedure between (1) and (2) terminates.

Proof. Assume that (2) does not occur. Then, $f_{s}^{-1}\left(x^{\prime}\right)$ is an immediate successor of $r$ in $T, f_{s}^{-1}\left(z^{\prime}\right)$ is an immediate successor of $f_{s}^{-1}\left(y^{\prime}\right), T\left[f_{s}^{-1}\left(x^{\prime}\right)\right] \equiv I_{0}, T\left[f_{s}^{-1}\left(y^{\prime}\right)\right] \equiv J$, and $T\left[f_{s}^{-1}\left(z^{\prime}\right)\right] \equiv J_{0}$. Because $T\left[f_{s}^{-1}\left(x^{\prime}\right)\right] \equiv I_{0}$, we can fix an embedding $\psi: T\left[f_{s}^{-1}\left(x^{\prime}\right)\right] \hookrightarrow I_{0}$. We have already observed that $\psi\left(f_{s}^{-1}\left(x^{\prime}\right)\right)$ is the root of $I_{0}$. Because $T\left[f_{s}^{-1}\left(y^{\prime}\right)\right] \equiv J$, we know that $f_{s}^{-1}\left(y^{\prime}\right)$ has infinitely many successor trees of type $J_{1}$. Since $T\left[f_{s}^{-1}\left(z^{\prime}\right)\right] \equiv J_{0}$, we know that $T\left[f_{s}^{-1}\left(z^{\prime}\right)\right] \hookrightarrow J_{1}$.

Consider the restriction of $\psi$ to the finite tree $T_{s}\left[f_{s}^{-1}\left(x^{\prime}\right)\right]$. Let $a$ be a node at level 1 in $T\left[f_{s}^{-1}\left(y^{\prime}\right)\right]$ which has type $J_{1}$ and is not in $T_{s}\left[f_{s}^{-1}\left(y^{\prime}\right)\right]$. Fix an embedding $\xi$ of $T_{s}\left[f_{s}^{-1}\left(z^{\prime}\right)\right]$ into $T[a]$ which sends $f_{s}^{-1}\left(z^{\prime}\right)$ to $a$ and let $\psi(a)=b \in I$. Notice that $J_{1} \hookrightarrow I[b]$ and $\psi \xi$ maps $T_{s}\left[f_{s}^{-1}\left(z^{\prime}\right)\right]$ into $I[b]$ with $\psi\left(\xi\left(f_{s}^{-1}\left(z^{\prime}\right)\right)\right)=b$. Define $\psi^{\prime}$ on $T_{s}\left[f_{s}^{-1}\left(x^{\prime}\right)\right]$ by making it equal to $\psi$ for all nodes that are not in $T_{s}\left[f_{s}^{-1}\left(z^{\prime}\right)\right]$ and equal to $\psi \xi$ on all nodes in $T_{s}\left[f_{s}^{-1}\left(z^{\prime}\right)\right]$. Because $f_{s}$ is an isomorphism between the finite trees $T_{s}^{\prime}\left[x^{\prime}\right]$ and $T_{s}\left[f_{s}^{-1}\left(x^{\prime}\right)\right]$, we can abuse notation and view $\psi^{\prime}$ as an embedding from $T_{s}^{\prime}\left[x^{\prime}\right]$ into $I_{0}$. Notice that $\psi^{\prime}$ has exactly the properties required for condition (1).

Sublemma 3.12 Each $\mathcal{R}_{e}$ requirement only acts finitely often.

Proof. Let $s$ be a stage after which all $R_{i}$ for $i<e$ do not act. In particular, they do not create new copies of $I_{0}$, so the number of witness tuples required by $\mathcal{R}_{e}$ is fixed at this stage. Let $t \geq s$ be a stage at which $\mathcal{R}_{e}$ has a full set of witness tuples and each such tuple is actually special in $T$. Suppose $\mathcal{R}_{e}$ acts with the tuple $\langle x, y, z\rangle$ after stage $t$. Then $\mathcal{R}_{e}$ declares itself satisfied with this tuple and remains satisfied forever since $\langle x, y, z\rangle$ is never taken away. Therefore, $\mathcal{R}_{e}$ acts at most once after stage $t$.

Sublemma 3.13 For each $u \in T, f_{s}(u)$ reaches a limit as $s \rightarrow \infty$.

Proof. The value of $f_{s}(u)$ can only change if some requirement $\mathcal{R}_{e}$ diagonalizes using a witness $x$ such that $f_{s}^{-1}\left(\varphi_{e}(x)\right) \prec u$. However, only requirements $\mathcal{R}_{e}$ with $e \leq u$ can act in this way. Therefore, once these requirements have stopped acting, the value of $f_{s}(u)$ cannot change.

Sublemma 3.14 Each requirement $\mathcal{R}_{e}$ is eventually satisfied. 
Proof. Let $n$ be the number of copies of $I_{0}$ created during the construction by requirements of higher priority. Let $s$ be a stage at which $\mathcal{R}_{e}$ has been assigned its final set of tuples, $\left\langle x_{i}, y_{i}, z_{i}\right\rangle$ for $i<e+n+1$, and all requirements of higher priority have stopped acting. For a contradiction, assume that $\varphi_{e}$ is an isomorphism from $T$ to $T^{\prime}$. Let $t \geq s$ be a stage at which $\varphi_{e}$ has converged on all entries in the tuples assigned to $\mathcal{R}_{e}$. Let $\left\langle x_{i}^{\prime}, y_{i}^{\prime}, z_{i}^{\prime}\right\rangle$ denote these image tuples. Since $\mathcal{R}_{e}$ did not get an easy win, we can assume that the values in these image tuples are either in the range of $f_{t}$ or in a copy of $I_{0}$ constructed by stage $t$.

For any tuple $\left\langle x_{i}, y_{i}, z_{i}\right\rangle$ which is mapped by $\varphi_{e}$ into a copy of $I_{0}$, the image tuple $\left\langle x_{i}^{\prime}, y_{i}^{\prime}, z_{i}^{\prime}\right\rangle$ is special in $T^{\prime}$ since $\varphi_{e}$ is an isomorphism. Also, since the witness tuples for $\mathcal{R}_{e}$ have distinct first components, if two witness tuples are mapped to copies of $I_{0}$, then these copies are distinct (or else we win trivially). Therefore, if at least $n+1$ many tuples are mapped to copies of $I_{0}$, then at least one of these copies of $I_{0}$ was not created by a requirement of higher priority. In this case, we immediately diagonalize with such a tuple and $\mathcal{R}_{e}$ is won permanently.

Otherwise, there are at least $e+1$ many witness tuples whose images lie in the range of $f_{t}$. It is possible that some requirements of lower priority will act in a manner which causes some of these witness tuples $\left\langle x_{i}^{\prime}, y_{i}^{\prime}, z_{i}^{\prime}\right\rangle$ to be contained in a copy of $I_{0}$ in $T^{\prime}$ at a later stage. If ever we reach a point where $n+1$ of the witness tuples are in copies of $I_{0}$, then $\mathcal{R}_{e}$ wins as in the previous paragraph.

If this does not happen, then for at least $e+1$ many tuples the values of $f^{-1}$ on each entry in $\left\langle x_{i}^{\prime}, y_{i}^{\prime}, z_{i}^{\prime}\right\rangle$ is not changed by any requirement of lower priority. Each of these tuples sits in a distinct cone immediately above the root of $T^{\prime}$. Since the requirements $\mathcal{N}_{u}$ for $u<e$ can only protect $e$ many of these cones, there must be an unprotected image tuple with which $\mathcal{R}_{e}$ enters the concurrent search of conditions (1) and (2). Because $\varphi_{e}$ is an isomorphism, by the $\Delta_{2}^{0}$ approximation to special tuples, $\mathcal{R}_{e}$ must eventually see that $\left\langle f_{t}^{-1}\left(x_{i}^{\prime}\right), f_{t}^{-1}\left(y_{i}^{\prime}\right), f_{t}^{-1}\left(z_{i}^{\prime}\right)\right\rangle$ is special. From here, $\mathcal{R}_{e}$ will begin the concurrent search procedure and must discover an embedding as in condition (1). At this point, $\mathcal{R}_{e}$ diagonalizes, contradicting the fact that $\varphi_{e}$ is an isomorphism. This finishes the proof of Lemma 3.10.

Lemma 3.15 Let $T$ be a tree of finite height, $\omega$-branching at its root $r$, such that all nodes above $r$ are of finite type. Let $x_{0}, x_{1}, \ldots$ be the immediate successors of $r$ in $T$. If $I_{0}$ is an isomorphism type such that infinitely many $i$ satisfy $T\left[x_{i}\right] \hookrightarrow I_{0}$ and infinitely many $j$ satisfy both $I_{0} \hookrightarrow T\left[x_{j}\right]$ and $T\left[x_{j}\right] \nrightarrow I_{0}$, then $T$ is not computably categorical.

Proof. Let $\mathcal{T}$ be the set of all isomorphism types of successor trees above $r$ in $T$, let $\mathcal{F} \subset \mathcal{T}$ be the set of types which do not embed into $I_{0}$ and let $\mathcal{E}$ contain those types in $\mathcal{F}$ into which $I_{0}$ embeds. By Corollary 3.8, $\mathcal{E}$ and $\mathcal{F}-\mathcal{E}$ each has a finite set of minimal elements, which we denote by $\mathcal{E}_{0}$ and $\mathcal{F}_{0}$, with every element of $\mathcal{E}$ lying above an element of $\mathcal{E}_{0}$ (in the embeddability order), and every element of $\mathcal{F}-\mathcal{E}$ lying above an element of $\mathcal{F}_{0}$. (Notice that no element of $\mathcal{E}$ can lie below an element of $\mathcal{F}-\mathcal{E}$, but it is possible for an element of $\mathcal{F}-\mathcal{E}$ to lie below an element of $\mathcal{E}$.) Lemma 3.3 yields a finite collection of finite subtrees, one $S_{i}$ in each $J_{i} \in \mathcal{F}_{0}$ and one $R_{i}$ in each $K_{i} \in \mathcal{E}_{0}$, such that no $S_{i}$ embeds into any other $S_{j}$ or into 
$I_{0}$, and no $R_{i}$ embeds into any other $R_{j}, S_{j}$ or $I_{0}$. The important facts about these relations are that for all $I \in \mathcal{T}$,

$$
\begin{aligned}
I \in \mathcal{F} & \Leftrightarrow \exists S_{i}\left(S_{i} \hookrightarrow I\right) \text { or } \exists R_{i}\left(R_{i} \hookrightarrow I\right) \\
& \exists R_{i}\left(R_{i} \hookrightarrow I\right) \Leftrightarrow I \in \mathcal{E} .
\end{aligned}
$$

At each stage $s$, we define the witness elements $w_{0, s}<\cdots<w_{p_{s}, s}$ at that stage to be those nodes $x \in T_{s}$ which we currently think are at the base of a successor tree of the root in $T$ whose isomorphism type is not in $\mathcal{F}$. More specifically, we look for $x$ satisfying:

- $x$ is an immediate successor of $r$ in $T_{s}$; and

- No $S_{i} \hookrightarrow T_{s}[x]$; and

- No $R_{i} \hookrightarrow T_{s}[x]$.

Then for each $e, w_{e}=\lim _{s} w_{e, s}$ exists, since infinitely many successor trees embed into $I_{0}$. We assign $e+1$ many witnesses to the requirement $\mathcal{R}_{e}$. (If there are not $e+1$ many witnesses available for $\mathcal{R}_{e}$, then it is not assigned any witnesses.) Because the limit of $w_{e, s}$ exists for each $e$, each requirement will eventually be assigned a full set of witnesses which never change. We need $e+1$ many witnesses since at most one witness may be forbidden by each of the requirements $\mathcal{N}_{u}$ for $u<e$.

We build $T^{\prime}$ in stages as $T_{s}^{\prime}$, and we build a $\Delta_{2}^{0}$-isomorphism $f: T \rightarrow T^{\prime}$ by finite approximations $f_{s}: T_{s} \rightarrow T_{s}^{\prime}$. At stage $s+1$, we extend $f_{s}$ to $f_{s+1}$ by adding fresh elements to $T_{s+1}^{\prime}$, unless the following conditions hold for some requirement $\mathcal{R}_{e}$ and one of its witnesses $w_{e, s}$. (Here we abuse notation slightly by letting $w_{e, s}$ stand for an arbitrary witness node for $\mathcal{R}_{e}$ at stage $s$. This conflicts with our indexing of the witness nodes above, but it makes the connection between $w_{e, s}$ and the requirement $\mathcal{R}_{e}$ clearer.)

- $\varphi_{e, s}\left(w_{e, s}\right) \downarrow \in T_{s}^{\prime}$; and

- $\varphi_{e, s}\left(w_{e, s}\right)$ is an immediate successor of $f_{s}(r)$ in $T_{s}^{\prime}$; and

- No $S_{i} \hookrightarrow T_{s}^{\prime}\left[\varphi_{e, s}\left(w_{e, s}\right)\right]$; and

- No $R_{i} \hookrightarrow T_{s}^{\prime}\left[\varphi_{e, s}\left(w_{e, s}\right)\right]$; and

- it is not the case that $f_{s}^{-1}\left(\varphi_{e, s}\left(w_{e, s}\right)\right) \prec u$ for any $u \in T$ with $u \leq e$ (this represents the restraint placed on $\mathcal{R}_{e}$ by $\mathcal{N}_{u}$ for $\left.u<e\right)$.

If these conditions hold for some $e \leq s$, then let $e$ be the highest priority requirement for which these conditions hold. We attempt to diagonalize to meet $\mathcal{R}_{e}$ by searching for a stage $t>s$ such that either

1. $f_{s}^{-1}\left(\varphi_{e}\left(w_{e, s}\right)\right)$ is not an immediate successor of $r$ in $T_{t}$; or 
2. Some $S_{i} \hookrightarrow T_{t}\left[f_{s}^{-1}\left(\varphi_{e}\left(w_{e, s}\right)\right)\right]$; or

3. Some $R_{i} \hookrightarrow T_{t}\left[f_{s}^{-1}\left(\varphi_{e}\left(w_{e, s}\right)\right)\right]$; or

4. There is an immediate successor $x$ of $r$ in $T_{t}$ such that $T_{t}[x] \cap T_{s}=\emptyset$ and $T_{s}^{\prime}\left[\varphi_{e}\left(w_{e, s}\right)\right] \hookrightarrow$ $T_{t}[x]$ and also some $R_{i} \hookrightarrow T_{t}[x]$.

If any of the first three conditions hold, then we define $f_{s+1}$ and $T_{s+1}^{\prime}$ as if no requirement needed attention. In this case, it appears that $\varphi_{e}$ is not an isomorphism since either $\varphi_{e}\left(w_{e, s}\right)$ is not the base of a successor tree in $T^{\prime}$ or $\varphi_{e}\left(w_{e, s}\right)$ is the base of a successor tree which does not embed into $I_{0}$. However, if $w_{e}=w_{e, s}$, then $w_{e}$ is the base of a successor tree in $T$ which does embed into $I_{0}$.

If the fourth condition holds, then we add $T_{t}[x]$ to our current copy of $T$, and define $f_{s+1}$ to map $T_{t}[x]$ onto $T_{s}^{\prime}\left[\varphi_{e}\left(w_{e, s}\right)\right]$, adding fresh elements to $T^{\prime}$ above $\varphi_{e}\left(w_{e, s}\right)$ to form a copy of $T_{t}[x]$. We also add more fresh elements to $T^{\prime}$ to be the new image of $T_{s}\left[f_{s}^{-1}\left(\varphi_{e}\left(w_{e}\right)\right)\right]$ under $f_{s+1}$. Thus $f_{s+1}$ is still an isomorphism, but hereafter $T^{\prime}\left[\varphi_{e}\left(w_{e, s}\right)\right]$ will grow as a copy of some successor tree containing $R_{i}$. By definition of $R_{i}$, this successor tree cannot be embedded into $I_{0}$. On the other hand, if $w_{e}=w_{e, s}$, then $T\left[w_{e}\right]$ can be embedded into $I_{0}$. Hence $\mathcal{R}_{e}$ will be satisfied.

If none of conditions 1-3 hold for any $t$, then $T\left[f_{s}^{-1}\left(\varphi_{e}\left(w_{e, s}\right)\right)\right]$ does not lie above any minimal element of $\mathcal{F}$, so it must embed into $I_{0}$. Since infinitely many successor trees above $r$ are supertrees of $I_{0}$ and do not embed into $I_{0}$, we see that condition 4 must then apply for some $t>s$. Therefore, this search procedure terminates.

If at some later stage $s^{\prime}$ we have $w_{e, s^{\prime}+1} \neq w_{e, s^{\prime}}$, then $\mathcal{R}_{e}$ and all lower priority requirements are injured at that stage. This happens if $w_{e, s^{\prime}}$ is no longer an immediate successor of $r$, or if some $R_{i}$ or $S_{i}$ embeds into $T_{s^{\prime}}\left[w_{e, s^{\prime}}\right]$. However, such injuries can only happen finitely often for each $e$, since $w_{e, s}$ converges. Therefore, each requirement $\mathcal{R}_{e}$ only acts finitely often.

Since $\mathcal{R}_{e}$ has $e+1$ many witnesses and the requirements $\mathcal{N}_{u}$ for $u<e$ protect at most $e$ many successor trees of the root in $T$ from having the value of $f_{s}$ changed on them, $\mathcal{R}_{e}$ must have some witness for which it is allowed to redefine $f_{s}$ if it needs to in order to diagonalize. (As in the previous construction, if $\varphi_{e}$ is not one-to-one or does not respect the ordering, then we win trivially and we cease trying to diagonalize.) Therefore, every requirement of the form $\mathcal{R}_{e}$ is eventually satisfied. Finally, notice that $f_{s}^{-1}(y)$ only changes if $y \in T_{s}^{\prime}\left[\varphi_{e, s}\left(w_{e, s}\right)\right]$ and $\mathcal{R}_{e}$ acts at stage $s$. In this case, some $R_{i} \hookrightarrow T_{s+1}^{\prime}\left[\varphi_{e, s}\left(w_{e, s}\right)\right]$ and therefore no $\mathcal{R}_{k}$ strategy ever redefines $f_{t}^{-1}$ for $t>s$ on this subtree in an attempt to diagonalize again. Therefore, $f_{s}^{-1}(y)$ reaches a limit for each $y \in T^{\prime}$.

The tree $T^{\prime}$ built by this process is computable, and isomorphic to $T$, since at each stage we have a homomorphism $f_{s}$ from $T_{s}$ into $T_{s}^{\prime}$, with $f_{s}(r)=r$, whose range is all of $T_{s}^{\prime}$. Our construction makes clear that $f=\lim _{s} f_{s}$ exists, since each $\mathcal{R}_{e}$ requirement must respect the $\mathcal{N}_{u}$ requirements for $u<e$. This finishes the proof of Lemma 3.15.

Lemma 3.16 Let $T$ be a tree of finite height with root $r$, such that all nodes above $r$ are of finite type. Suppose there exist distinct isomorphism types $I_{0}, I_{1}, \ldots$ and $I_{\omega}$ appearing as 
successor trees above $r$. Suppose further that for every $i, I_{i} \hookrightarrow I_{\omega}$, and that $I_{\omega}$ appears infinitely often as a successor tree above $r$. Then $T$ is not computably categorical.

Notice that we do not require that $I_{0}, I_{1}, \ldots I_{\omega}$ be the only isomorphism types appearing as successor trees above $r$.

Proof. First we apply Corollary 3.8 to the set of successor trees above $r$ which do not embed into $I_{\omega}$, and use Lemma 3.3 to choose finite subtrees $R_{1}, \ldots R_{m}$ of the minimal elements of this set, such that no $R_{j}$ embeds into $I_{\omega}$.

Case 1. If there are only finitely many $i$ such that $I_{i} \hookrightarrow I_{\omega} \hookrightarrow I_{i}$, then we will appeal to Lemma 3.19. Since there are finitely many such $I_{i}$, there is a finite subtree $S \subseteq I_{\omega}$ such that $S \hookrightarrow I_{i}$ for any such $I_{i}$. Hence the immediate successors $x$ of $r$ such that $T[x] \equiv I_{\omega}$ are precisely those $x$ satisfying:

- $(\forall s)(\forall j) R_{j} \nrightarrow T_{s}[x]$; and

- $(\exists s) S \hookrightarrow T_{s}[x]$.

This set is $\Delta_{2}^{0}$ and infinite, and for all $x_{0}$ and $x_{1}$ in the set we have $T\left[x_{0}\right] \hookrightarrow I_{\omega} \hookrightarrow T\left[x_{1}\right]$, so indeed Lemma 3.19 will apply. (Also, the proof of Lemma 3.19 will not depend on Lemma 3.16 at all.)

Case 2. Now suppose that there are infinitely many $i$ such that $I_{i} \hookrightarrow I_{\omega} \hookrightarrow I_{i}$. In this case, we build $T^{\prime}$ and an embedding $f: T \rightarrow T^{\prime}$ such that $T^{\prime}$ is equal to the range of $f$ plus extra copies of $I_{\omega}$ added as immediate successors of the root. Because $I_{\omega}$ occurs infinitely often as a successor tree of the root in $T$, we have that $T$ and $T^{\prime}$ are isomorphic. As before, we build $T^{\prime}$ and $f$ in stages such that at each stage $s, T_{s}^{\prime}$ is equal to the range of $f_{s}$ plus finitely many copies of $I_{\omega}$.

We pick one immediate successor $y_{0}$ of $r$ in $T$ such that $T\left[y_{0}\right] \cong I_{\omega}$, and use this finite information to identify our witness nodes. Our goal is to identify witness nodes $x \in T$ such that $T[x] \hookrightarrow I_{\omega} \hookrightarrow T[x]$ and then to diagonalize by making $T^{\prime}\left[\varphi_{e}(x)\right] \cong I_{\omega}$. Because $T[x] \hookrightarrow I_{\omega}$ if and only if for each $R_{i}, R_{i} \leftrightarrow T[x]$, we can measure that $x$ is an immediate successor of the root such that $T[x] \hookrightarrow I_{\omega}$ in a $\Delta_{2}^{0}$ way. To measure whether $I_{\omega} \hookrightarrow T[x]$, at stage $s$, for each $x$ which is an immediate successor of $r$ in $T_{s}$, we define

$$
t(x, s)=\mu t\left[t \geq x \& T_{t}\left[y_{0}\right] \nrightarrow T_{s}[x]\right]
$$

Then we choose the witness nodes $w_{0, s}, \ldots w_{p_{s}, s}$ to be those $x$ which are successors of the root in $T_{s}$ (and hence for which $t(x, s)$ is defined) and no $R_{j}$ embeds into $T_{s}[x]$. We index these witnesses so that

$$
t\left(w_{0, s}, s\right) \leq t\left(w_{1, s}, s\right) \leq \cdots \leq t\left(w_{p_{s}, s}, s\right)
$$

with $w_{e, s}<_{\mathbb{N}} w_{e+1, s}$ for any $e$ such that $t\left(w_{e, s}, s\right)=t\left(w_{e+1, s}, s\right)$.

Clearly, if $x$ appears as a witness node at infinitely many stages $s$, then $x$ must be an immediate successor of $r$ and $T[x] \hookrightarrow I_{\omega}$. Moreover, for an immediate successor $x$ such that 
$I_{\omega} \hookrightarrow T[x]$, we must have $\lim _{s} t(x, s)=\infty$. On the other hand, if $T[x]$ is of one of the infinitely many types $I_{i}$ for which $I_{\omega} \nsucc I_{i}$, then $I_{\omega} \nsucc T[x]$, and there is some $t$ and some finite tree $S_{i}$ such that $S_{i} \subseteq T_{t}\left[y_{0}\right]$ and $S_{i} \hookrightarrow T[x]$. Therefore, $\lim _{s} t(x, s) \downarrow \leq t$. Hence for each of these latter values of $x$, there must be an $e$ with $\lim _{s} w_{e, s} \downarrow=x$. We write $w_{e}$ for this $x$, and note that since there are infinitely many such $x$, the limit $w_{e}$ must exist for all $e$. This gives us our witness nodes. We assign $e+1$ many witness to the requirement $\mathcal{R}_{e}$. (As before, we abuse notation when we describe the action of requirement $\mathcal{R}_{e}$ at stage $s$ by denoting its witness by $w_{e, s}$.)

At stage $s+1$ of the construction, we extend our previous map $f_{s}$ to $T_{s+1}$. We do this by adding fresh elements to the image $T_{s+1}^{\prime}$, unless some requirement $\mathcal{R}_{e}$ requires attention. We say that $\mathcal{R}_{e}$ requires attention if there exists a witness $w_{e, s}$ for $\mathcal{R}_{e}$ such that $\varphi_{e, s}\left(w_{e, s}\right) \downarrow$ (say $\left.\varphi_{e, s}\left(w_{e, s}\right)=y\right), y$ is an immediate successor of the root in $T_{s}^{\prime}, y$ is in the range of $f_{s}$, and it is not the case that $f_{s}^{-1}(y) \prec u$ for some $u<e$. Notice that if $\mathcal{R}_{e}$ does not require attention because $y$ is not an immediate successor of the root in $T^{\prime}$, then we win $\mathcal{R}_{e}$ trivially as long as $w_{e, s}=w_{e}$. If $y$ is a successor of the root of $T_{s}^{\prime}$ but $\mathcal{R}_{e}$ does not require attention because $y$ is not in the range of $f_{s}$, then $y$ is the root of a tree of type $I_{\omega}$ in $T^{\prime}$. Again, if $w_{e, s}=w_{e}$, then we have won $\mathcal{R}_{e}$ trivially. And if $y \prec u$ for some $u<e$, then we do not allow $\mathcal{R}_{e}$ to act on this witness $w_{e, s}$ since the action could damage the negative requirement $\mathcal{N}_{u}$.

If $\mathcal{R}_{e}$ is the highest priority requirement needing attention, then we check if some $R_{j}$ embeds into $T_{s}^{\prime}[y]$. If so, then we know $T_{s}^{\prime}[y] \nrightarrow I_{\omega}$. Assuming $w_{e, s}$ turns out to be a true witness, $T\left[w_{e, s}\right] \neq T^{\prime}[y]$ and we have won $\mathcal{R}_{e}$. If no $R_{j}$ embeds in $T_{s}^{\prime}[y]$, then we attempt to diagonalize. Search concurrently until we find

1. some $R_{j} \hookrightarrow T_{t}\left[f_{s}^{-1}(y)\right]$ for $t \geq s$; or

2. some witness $w_{0, t}, \ldots, w_{e, t}$ changes; or

3. an embedding of $T_{s}^{\prime}[y]$ into $I_{\omega}$ appears.

By the definition of $R_{j}$, we know that this search procedure must terminate.

If the search in (1) or (2) is successful, then we do not need to do anything for $\mathcal{R}_{e}$. Either we do not really believe that $w_{e, s}$ is the correct witness, or we believe that when we get to stage $t$ we will win $\mathcal{R}_{e}$ easily because $R_{j} \hookrightarrow T_{t}^{\prime}[y]$.

If the search in (3) is successful, then we add fresh elements to $T_{s+1}^{\prime}$ above $y$ to make $T_{s+1}^{\prime}[y] \cong I_{\omega}$. (Notice that since $I_{\omega}$ is of finite type, we can execute this step all at once, using a nice copy of $I_{\omega}$ constructed from only finitely much information.) Also, add more fresh elements to $T_{s+1}^{\prime}$ to be the new image of $T_{s}\left[f_{s}^{-1}(y)\right]$ under $f_{s+1}$. Thus, we have redefined $f$ on $T_{s}\left[f_{s}^{-1}(y)\right]$, but as in the previous arguments, because $\mathcal{R}_{e}$ must respect $\mathcal{N}_{u}$ for $u<e$, this can happen only finitely often. Moreover, if $w_{e}=w_{e, s}$, then $T\left[w_{e}\right]$ will not be isomorphic to $I_{\omega}$, hence not isomorphic to $T\left[\varphi_{e}\left(w_{e}\right)\right]$, satisfying requirement $\mathcal{R}_{e}$. Finally, notice that for $y \in T^{\prime}$, we only change $f_{s}^{-1}(y)$ when we remove $y$ from the range of $f$. However, when this happens, $y$ permanently becomes part of an auxiliary copy of $I_{\omega}$. 
The next lemma is not a separate case of our overall proof of Proposition 3.1, but it will be used later in the proof of Lemma 3.18.

Lemma 3.17 Let $T$ be a tree of finite height with root $r$. Let $x_{0}, x_{1}, \ldots$ be the immediate successors of $r$ in $T$, and assume that every $x_{i}$ is of finite type. Moreover, assume that there exists an infinite $\Delta_{2}^{0}$ set $G \subseteq\left\{x_{i}: i \in \omega\right\}$ such that

1. if $x_{i} \in G$ and $T\left[x_{i}\right] \cong T\left[x_{j}\right]$, then $x_{j} \in G$,

2. every $T\left[x_{i}\right]$ with $x_{i} \in G$ embeds into infinitely many $T\left[x_{j}\right]$ with $x_{j} \in G$, and

3. for each $x_{i} \in G,\left\{x_{j}: T\left[x_{j}\right] \cong T\left[x_{i}\right]\right\}$ is a finite subset of $G$.

Then $T$ is not computably categorical.

Proof. We construct a computable tree $T^{\prime}$ isomorphic to $T$, such that for every $e$, if $\varphi_{e}$ were an isomorphism from $T$ to $T^{\prime}$, then one of the $\cong$-classes described in the lemma would be infinite. Let $G_{s}$ be a computable approximation to $G$, with every $G_{s}$ finite. At each stage $s$ we define a finite subtree $D_{s} \subset T$ with $D_{s} \subseteq D_{s+1}$ and an isomorphism $f_{s}: D_{s} \rightarrow T_{s}^{\prime}$, such that $f_{s}$ converges to a $\Delta_{2}^{0}$-isomorphism $f: T \rightarrow T^{\prime}$. We will choose infinitely many witness elements $w_{i}^{j}$ for each (total one-to-one) function $\varphi_{i}$.

We begin by motivating our strategy for a single $\mathcal{R}_{e}$ requirement. For simplicity of notation, we assume that $G$ is computable, as adding the $\Delta_{2}^{0}$ approximation to $G$ is straightforward. $\mathcal{R}_{e}$ begins with a single witness $w_{e}^{0} \in G$ and waits for a stage $s$ such that $\varphi_{e}\left(w_{e}^{0}\right)$ converges. If $\varphi_{e}\left(w_{e}^{0}\right) \in T_{s}^{\prime}$ and $f_{s}^{-1}\left(\varphi_{e}\left(w_{e}^{0}\right)\right) \notin G$, then $\mathcal{R}_{e}$ is satisfied and we do not perform any action. (Notice that by condition (1) on $G$, if some $x \in G$ we have $f^{-1}\left(\varphi_{e}(x)\right) \notin G$, then $\varphi_{e}$ cannot be an isomorphism from $T$ to $T^{\prime}$.) Otherwise, if either $\varphi_{e}\left(w_{e}^{0}\right) \notin T_{s}^{\prime}$ or $f_{s}^{-1}\left(\varphi_{e}\left(w_{e}^{0}\right)\right) \in G$, we begin our action for $\mathcal{R}_{e}$.

Search for a $t>s$ and an $x \in T$ such that $x \in G, T_{s}^{\prime}\left[\varphi_{e}\left(w_{e}^{0}\right)\right] \hookrightarrow T_{t}[x]$, and $T_{t}[x]$ is disjoint from $D_{s}$. If $f_{s}^{-1}\left(\varphi_{e}\left(w_{e}^{0}\right)\right)$ is in $G$, then we must find such an $x$ since $T\left[f_{s}^{-1}\left(\varphi_{e}\left(w_{e}^{0}\right)\right)\right]$ embeds into $T[y]$ for infinitely many $y \in G$. (If $\varphi_{e}\left(w_{e}^{0}\right) \notin T_{s}^{\prime}$, then the embedding condition is trivial and we merely look for $T_{t}[x]$ which is disjoint from $D_{s}$.) We now define the map $f_{s+1}$ by changing the map $f_{s}$ on $T_{s}\left[f_{s}^{-1}\left(\varphi_{e}\left(w_{e}^{0}\right)\right)\right]$. Use the embedding of $T_{s}^{\prime}\left[\varphi_{e}\left(w_{e}^{0}\right)\right]$ into $T_{t}[x]$ and add extra elements to $T_{s+1}^{\prime}$ to make $f_{s+1}$ map $T_{t}[x]$ onto $T_{s+1}^{\prime}\left[\varphi_{e}\left(w_{e}^{0}\right)\right]$. Add more new elements to $T_{s+1}^{\prime}$ to serve as the new image of $T_{s}\left[f_{s}^{-1}\left(\varphi_{e}\left(w_{e}^{0}\right)\right)\right]$ under $f_{s+1}$. For all other points in $D_{s}$, let $f_{s+1}=f_{s}$. We now have defined $f_{s+1}$ on $D_{s+1}$, which is equal to $D_{s}$ plus $T_{t}[x]$. Finally, we define $w_{e}^{1}=x$. (Notice that we can speed up the approximation of $T$ to assume that $D_{s+1} \subseteq T_{s+1}$.)

Repeat the above procedure, but working with $w_{e}^{1}$ instead of $w_{e}^{0}$. Notice that if we keep extending our map $f_{s+1}$ to copy the successor trees it is currently defined on, we will get that $f$ is an isomorphism between $T^{\prime}\left[\varphi_{e}\left(w_{e}^{0}\right)\right]$ and $T\left[w_{e}^{1}\right]$. Therefore, if $\varphi_{e}$ is an isomorphism,

$$
T\left[w_{e}^{0}\right] \cong T^{\prime}\left[\varphi_{e}\left(w_{e}^{0}\right)\right] \cong T\left[w_{e}^{1}\right]
$$


By repeating this process (and assuming that $\varphi_{e}$ continues to converge on all of our witnesses $w_{e}^{n}$ and is well behaved - see below), we get a sequence of witnesses $w_{e}^{n}$ such that if $\varphi_{e}$ is an isomorphism, then $T\left[w_{e}^{n}\right] \cong T\left[w_{e}^{n+1}\right]$ for all $n$. This contradicts the fact that the isomorphism types given by nodes in $G$ occur finitely often. Notice that we change the map $f$ as we go from $f_{s}$ to $f_{s+1}$ on $T_{s}\left[f_{s}^{-1}\left(\varphi_{e}\left(w_{e}^{n}\right)\right)\right]$ and we also change the map from $f_{s}^{-1}$ to $f_{s+1}^{-1}$ on $T_{s}^{\prime}\left[\varphi_{e}\left(w_{e}^{n}\right)\right]$. Since we are not turning $T_{s}^{\prime}\left[\varphi_{e}\left(w_{e}^{n}\right)\right]$ into an auxiliary tree, we will need to explicitly address the requirements $\mathcal{M}_{u}$ for the first time.

By well behaved, we mean that $\varphi_{e}$ is one-to-one, that it maps the root to the root, that it maps comparable nodes to comparable nodes, and that it maps incomparable nodes to incomparable nodes. If we ever see any of these conditions violated, then we know $\varphi_{e}$ is not an isomorphism and we can stop working on $\mathcal{R}_{e}$. In all of the work below, we assume that we stop work on $\mathcal{R}_{e}$ if we get an easy win because it is not well behaved in this sense.

Combining the basic strategy for $\mathcal{R}_{e}$ with the $\mathcal{N}_{i}$ strategies is a little more subtle than in previous constructions because one $\mathcal{R}_{e}$ requirement can cause infinitely many changes in the map $f_{s}$. Before redefining $f_{s}$ on $T_{s}\left[f_{s}^{-1}\left(\varphi_{e}\left(w_{e}^{n}\right)\right)\right]$, we check if $f_{s}^{-1}\left(\varphi_{e}\left(w_{e}^{n}\right)\right) \prec m$ for any $m$ from $0, \ldots,\langle e, n\rangle$ in $T$. If not, then we act as above. If it is below any such $m$, then we cannot redefine $f_{s}$ on this subtree. We also employ a similar strategy to deal with the $\mathcal{M}_{i}$ strategies. That is, if $\varphi_{e}\left(w_{e}^{n}\right) \leq\langle e, n\rangle$, then $\mathcal{R}_{e}$ cannot use $w_{e}^{n}$ as a witness since this would involve redefining $f_{s}^{-1}\left(\varphi_{e}\left(w_{e}^{n}\right)\right)$. In either case, $\mathcal{R}_{e}$ must repick its witness $w_{e}^{n}$. If $n>0$, we declare that $w_{e}^{n}$ is undefined. This forces us to repeat the cycle above for $w_{e}^{n-1}$ and gives us a new (large) witness $w_{e}^{n}$. Assuming that $\varphi_{e}$ is well behaved, this new witness gives us a different value for $f_{s}^{-1}\left(\varphi_{e}\left(w_{e}^{n}\right)\right.$ ) (which we assume is in $G$ ). Since each point in $G$ is an immediate successor of the root in $T$, we will have to repeat this process at most $2\langle e, n\rangle+1$ many times before we are guaranteed to be allowed to redefine $f_{s}$. If $n=0$, then we need to choose a new initial witness $w_{e}^{0}$. To do this, we declare that the old $w_{e}^{0}$ is disallowed for $\mathcal{R}_{e}$ and we let the new $w_{e}^{0}$ be the least element of $G$ which has not been disallowed for $\mathcal{R}_{e}$. If $\varphi_{e}$ is well behaved, then we will have to redefine our initial witness in this fashion at most $2\langle e, 0\rangle+1$ many times. Therefore, we eventually get our infinite sequence of witnesses and win.

We also need to see how different $\mathcal{R}$ strategies work together. Each $\mathcal{R}_{e}$ will have some finite (possibly empty) list of witnesses $w_{e}^{0}, \ldots, w_{e}^{n}$ at stage $s$. We say that $w_{j}^{i}$ has higher priority than $w_{q}^{p}$ if $\langle i, j\rangle\left\langle\langle p, q\rangle\right.$. Consider an $\mathcal{R}_{e}$ strategy working with other $\mathcal{R}$ strategies. If $\mathcal{R}_{e}$ has a largest witness $w_{e}^{n}$ and $\varphi_{e}\left(w_{e}^{n}\right)$ converges with $f_{s}^{-1}\left(\varphi_{e}\left(w_{e}^{n}\right)\right) \in G$, then in addition to checking whether $f_{s}^{-1}\left(\varphi_{e}\left(w_{e}^{n}\right)\right)$ is below any of the numbers $0, \ldots,\langle e, n\rangle$ in $T, \mathcal{R}_{e}$ also checks whether $f_{s}^{-1}\left(\varphi_{e}\left(w_{e}^{n}\right)\right)$ is equal to any other $w_{i}^{m}$. If so, then changing $f_{s}$ on $T_{s}\left[f_{s}^{-1}\left(\varphi_{e}\left(w_{e}^{n}\right)\right)\right]$ could damage the requirement $\mathcal{R}_{i}$. Therefore, $\mathcal{R}_{e}$ checks if the node $w_{i}^{m}$ has higher priority than $w_{e}^{n}$. If so, then $\mathcal{R}_{e}$ cannot change the map on this cone, so it acts as when it was restricted by an $\mathcal{N}$ or $\mathcal{M}$ requirement. If not, then it causes all $w_{i}^{m}$ of lower priority to become undefined and goes ahead with its action as above.

Notice that this action may allow $\mathcal{R}_{i}$ to injure $\mathcal{R}_{e}$ even though $e<i$. However, only finitely many witnesses $w_{i}^{m}$ can injure a given $w_{e}^{n}$, and therefore, $w_{e}^{n}$ will eventually reach a 
limit which $\mathcal{R}_{e}$ can use.

We now present the full construction, which is nothing more than the above description with the $\Delta_{2}^{0}$ guessing for elements of $G$. We start by setting $D_{0}=\{r\}$ and $T_{0}^{\prime}=\{0\}$, with $f_{0}(r)=0$.

At stage $s+1$, we make a preliminary definition of our witnesses by induction on $i$ from $0 \leq i \leq s$. If $w_{i, s}^{0} \in G_{s}$, then let $w_{i, s+1}^{0}=w_{i, s}^{0}$ and $w_{i, s+1}^{j}=w_{i, s}^{j}$ for all $j>0$ such that $w_{i, s}^{j}$ is defined. If $w_{i, s}^{0} \notin G_{s}$ or $w_{i, s}^{0}$ is not defined, then $w_{i, s+1}^{0}$ and all lower priority witnesses $w_{e, s+1}^{n}$ are undefined (even if some of these were defined earlier in the induction). Next, we check if some new initial witness $w_{k, s+1}^{0}$ can be defined. If there is a $k \leq s$ and an element $x \in G_{s}$ such that $w_{k, s+1}^{0}$ is undefined, $x \notin\left\{w_{i, s+1}^{j}:\langle i, j\rangle<\langle k, 0\rangle\right\}$, and $x$ is not disallowed for $w_{k}^{0}$, then we let $w_{k, s+1}^{0}$ be the least such $x$. Make all lower priority witnesses undefined.

We then find the least pair $\langle i, j\rangle$ such that $\varphi_{i, s}$ is well behaved, $w_{i, s+1}^{j}$ is defined, $w_{i, s+1}^{j+1}$ is not defined, $\varphi_{i, s}\left(w_{i, s+1}^{j}\right) \downarrow$, and either $f_{s}^{-1}\left(\varphi_{i, s}\left(w_{i, s+1}^{j}\right)\right) \in G_{s}$ or $\varphi_{i, s}\left(w_{i, s+1}^{j}\right) \notin T_{s}^{\prime}$. (If there is no such pair, we end the stage, let $D_{s+1}=D_{s} \cup\{s\}$ and $f_{s+1}=f_{s}$ plus add one fresh element $f_{s+1}(s)$ to $T_{s+1}^{\prime}$ if needed.) If $\varphi_{i, s}\left(w_{i, s+1}^{j}\right) \in T_{s}^{\prime}$, then check the following two conditions for compatibility with the appropriate $\mathcal{N}$ and $\mathcal{R}$ requirements. (In the case when $\varphi_{i, s}\left(w_{i, s+1}^{j}\right) \notin T_{s}^{\prime}$, we can skip these checks.)

First, check for compatibility with the $\mathcal{N}$ and $\mathcal{M}$ requirements. If $\varphi_{i}\left(w_{i, s+1}^{j}\right)>\langle i, j\rangle$ and there is no $k \leq\langle i, j\rangle$ such that $f_{s}^{-1}\left(\varphi_{i}\left(w_{i, s+1}^{j}\right)\right) \prec k$ in $T_{s}$, then go to the check in the next paragraph. Otherwise, if $j>0$, declare $w_{i, s+1}^{j}$ undefined and begin the next stage. If $j=0$, then declare $w_{i, s+1}^{0}$ disallowed for $w_{i}^{0}$, make $w_{i, s+1}^{0}$ undefined and go to the next stage. (In both of these cases, we extend $D_{s}$ to $D_{s+1}=D_{s} \cup\{s\}$, add an extra element to $T_{s+1}^{\prime}$, and define $f_{s+1}$ on this new element if necessary.)

Second, check for compatibility with the $\mathcal{R}$ requirements. If there is no higher priority $w_{e, s+1}^{n}$ such that $\varphi_{i}\left(w_{i, s+1}^{j}\right)=f_{s}\left(w_{e, s+1}^{n}\right)$, then go to the next paragraph. Otherwise, if there is such a $w_{e, s+1}^{n}$ and $j>0$, declare $w_{i, s+1}^{j}$ undefined and begin the next stage. If $j=0$, then declare $w_{i, s+1}^{0}$ disallowed for $w_{i}^{0}$, make $w_{i, s+1}^{0}$ undefined and go to the next stage. (Handle $D_{s+1}, T_{s+1}^{\prime}$ and $f_{s+1}$ as in the previous paragraph.)

If both of these checks are successful, search for the least stage $t>s+1$ such that either

- $\exists x \in G_{t}\left(T_{t}[x] \cap D_{s}=\emptyset \& T_{s}^{\prime}\left[\varphi_{i}\left(w_{i, s+1}^{j}\right)\right] \hookrightarrow T_{t}[x]\right)$; or

- $\varphi_{i, s}\left(w_{i, s+1}^{j}\right) \in T_{s}^{\prime}$ and $f_{s}^{-1}\left(\varphi_{i, s}\left(w_{i, s+1}^{j}\right)\right) \notin G_{t}$.

In the latter case we repeat the above process for the next pair $\langle i, j\rangle \leq s$ which appears to need attention. In the former case, we set $w_{i, s+1}^{j+1}=x$, add elements to $T_{s+1}^{\prime}$ above (and possibly including) the node $\varphi_{i}\left(w_{i, s+1}^{j}\right)$ to make a copy of $T_{t}[x]$, and define $f_{s+1}$ to map $T_{t}[x]$ onto these elements, according to the embedding we found. If this requires redefining $f_{s+1}$ on elements of $D_{s}$ which had mapped into $T_{s}^{\prime}\left[\varphi_{i}\left(w_{i, s+1}^{j}\right)\right]$ under $f_{s}$, we add fresh elements to $T_{s+1}^{\prime}$ to be their images. For all other elements of $D_{s}, f_{s+1}$ takes the same value as $f_{s}$. We add the elements of $T_{t}[x]$ to $D_{s+1}$ and we enumerate $s$ into $D_{s+1}$, adding a fresh element as its image in $T_{s+1}^{\prime}$ if necessary. Thus $T_{s+1}^{\prime}$ is the bijective image of $D_{s+1}$ under $f_{s+1}$. 
We claim that the search for stage $t$ must eventually terminate. If $f_{s}^{-1}\left(\varphi_{i}\left(w_{i, s+1}^{j}\right)\right) \notin G$, this is clear. If $f_{s}^{-1}\left(\varphi_{i}\left(w_{i, s+1}^{j}\right)\right) \in G$, then there are infinitely many other nodes $x \in G$ such that $T\left[f_{s}^{-1}\left(\varphi_{i}\left(w_{i, s+1}^{j}\right)\right)\right] \hookrightarrow T[x]$. Eventually we find a node $x$ (in $G_{t}$ but not necessarily in $G$ ) with such an embedding, such that $T[x] \cap D_{s}=\emptyset$, and we use it. Finally, if $\varphi_{i}\left(w_{i, s+1}^{j}\right) \notin T_{s}^{\prime}$, then $T_{s}^{\prime}\left[\varphi_{i}\left(w_{i, s+1}^{j}\right)\right]$ is considered to be empty, hence embeds trivially into $T_{t}[x]$ for the first $x \notin D_{s}$ to appear in any later $T_{t} \cap G_{t}$.

The verification that the construction succeeds is essentially as described in the informal setting. The witness $w_{0, s}^{0}$ can only be changed if it leaves $G_{s}$ or if $\varphi_{0}\left(w_{0, s}^{0}\right)$ converges either to 0 or such that $f_{s}^{-1}\left(\varphi_{0}\left(w_{0, s}^{0}\right)\right) \prec 0$ in $T$. Therefore, this witness is only injured finitely often due to the $\Delta_{2}^{0}$ nature of $G$ and is injured at most once by each of the requirements $\mathcal{N}_{0}$ and $\mathcal{M}_{0}$. Since no other requirement can injure $w_{0, s}^{0}$, this witness eventually reaches its final value. Similarly, for each $w_{i, s}^{j}$, once the higher priority witnesses have reached their final values (which may include never being defined again), this witness suffers finite injury due to the fact that $G$ is $\Delta_{2}^{0}$, finite injury due to the restraints of $\mathcal{N}$ and $\mathcal{M}$, and finite injury due to the restraints of the higher priority witnesses for $\mathcal{R}$ requirements. Therefore, for every witness $w_{i, s}^{j}$, there is a stage $t$ such that either $w_{i, s}^{j}$ has stabilized by stage $t$ or $w_{i, s}^{j}$ is never defined after stage $t$.

Since each witness stabilizes, $\mathcal{R}_{i}$ only changes $f_{s}$ finitely many times for each potential witness $w_{i, s}^{j}$. Therefore, because of the restraint imposed by the $\mathcal{N}$ requirements, $f(x)=$ $\lim _{s} f_{s}(x)$ exists for all $x \in T$ and because of the restraint of the $\mathcal{M}$ requirements, $f^{-1}(y)=$ $\lim _{s} f_{s}^{-1}(y)$ exists for all $y \in T^{\prime}$. Thus $f$ gives a $\Delta_{2}^{0}$-isomorphism from $T$ to $T^{\prime}$.

If $\varphi_{i}$ is indeed well behaved and total, then we define a growing sequence of nodes $w_{i, s}^{0}, \ldots, w_{i, s}^{j}$ which eventually settle down to $w_{i}^{0}, \ldots, w_{i}^{j}$. If $f^{-1}\left(\varphi_{i}\left(w_{i}^{j}\right)\right) \notin G$, then $\varphi_{i}$ cannot be an isomorphism, since the lemma assumes that if $T[x] \cong T[y]$, then $(x \in G \Longleftrightarrow y \in G)$. If $f^{-1}\left(\varphi_{i}\left(w_{i}^{j}\right)\right) \in G$, then eventually the second clause in the search for stage $t$ will never again apply, and we will find a $t$ and an $x$ which we define to be $w_{i}^{j+1}$.

Once $w_{i}^{j+1}$ has converged, we define $f_{s}\left(w_{i}^{j+1}\right)=\varphi_{i}\left(w_{i}^{j}\right)$. This action may be injured finitely many times, but eventually it settles on a final $w_{i}^{j+1}$ with $f\left(w_{i}^{j+1}\right)=\varphi_{i}\left(w_{i}^{j}\right)$. We know that $f$ is an isomorphism from $T$ to $T^{\prime}$. If $\varphi_{i}$ were an isomorphism as well, then we would have

$$
T\left[w_{i}^{j}\right] \cong T^{\prime}\left[\varphi_{i}\left(w_{i}^{j}\right)\right] \cong T\left[w_{i}^{j+1}\right]
$$

for every $j$, the first isomorphism being $\varphi_{i}$ and the second being $f^{-1}$. But $w_{i}^{0} \in G$ (since we check this immediately at every stage), and $w_{i}^{k} \neq w_{i}^{j}$ for all $k \neq j$, since each new $w_{i}^{k}$ is always chosen as a node in $T$ not yet in the domain $D_{s}$ of $f_{s}$. This contradicts the assumptions of the lemma, so $T$ and $T^{\prime}$ cannot be computably isomorphic.

Lemma 3.18 Let $T$ be a tree of finite height, such that the root $r$ has infinitely many immediate successors $x_{0}, x_{1}, \ldots$ Assume that all nodes above $r$ are of finite type, and that there are infinitely many isomorphism types in the set $\left\{T\left[x_{i}\right]\right\}$. Suppose that only finitely many of these isomorphism types appear infinitely often as successor trees above $r$, and that for each 
such type I, only finitely many other types appearing above $r$ embed into $I$. Then $T$ is not computably categorical.

Proof. Let $\mathcal{T}$ be the set of isomorphism types of successor trees in $T$ and let $\mathcal{I}$ be the set of types in $\mathcal{T}$ which embed in any of the infinite-occurring types (including the infinitelyoccurring types themselves). By assumption $\mathcal{I}$ is finite. We let $\left\{S_{i}\right\}$ be a finite collection of finite trees such that no $S_{i}$ embeds into any element of $\mathcal{I}$, yet every $T\left[x_{i}\right] \notin \mathcal{I}$ has some $S_{i}$ as a subtree. (Here we use Corollary 3.8 and Lemma 2.9.) The elements of $\mathcal{I}$ are precisely those types into which no $S_{i}$ embeds. Therefore, there is a $\Delta_{2}^{0}$ guessing process to identify all successor trees above $r$ whose type is in $\mathcal{I}$.

Notice further that by Corollary 3.6, we infer that of the elements of $\mathcal{T}-\mathcal{I}$, all but finitely many embed into infinitely many other elements of $\mathcal{T}-\mathcal{I}$. Let

$$
U=\left\{x_{i}: T\left[x_{i}\right] \in \mathcal{T}-\mathcal{I} \&(\exists m)(\forall j \geq m) T\left[x_{i}\right] \nrightarrow T\left[x_{j}\right]\right\}
$$

be this finite set. We will concern ourselves with the set $F$ of immediate successors $x$ of $r$ in $T$ such that $T[x] \in \mathcal{T}-\mathcal{I}$ and $T[x]$ embeds into infinitely many other elements of $\mathcal{T}-\mathcal{I}$. We have a $\Delta_{2}^{0}$-approximation $F_{s}$ for $F$, and we may assume that each $F_{s} \cap U=\emptyset$.

Case 1 . Suppose there are only finitely many equivalence classes $\mathcal{E}_{0}, \ldots \mathcal{E}_{p}$ under $\equiv$ among $\{T[x]: x \in F\}$. At least one must be infinite, so assume that $\mathcal{E}_{0}, \ldots \mathcal{E}_{q}$ are the infinite classes and $\mathcal{E}_{q+1}, \ldots, \mathcal{E}_{p}$ are the finite ones. Since each isomorphism type occurs only finitely often in each $\mathcal{E}_{i}$, we have that the set

$$
X=\left\{x \in F: T[x] \in \mathcal{E}_{q+1} \cup \cdots \cup \mathcal{E}_{p}\right\}
$$

is finite. Let $G=F-X$. Since $F$ is $\Delta_{2}^{0}$ and $X$ is finite, $G$ is $\Delta_{2}^{0}$. $G$ is exactly the kind of set to which we can apply Lemma 3.17. Therefore, $T$ is not computably categorical.

Case 2. Suppose there are infinitely many equivalence classes under $\equiv$ among $\{T[x]$ : $x \in F\}$. We will write $\mathcal{E}_{j} \hookrightarrow \mathcal{E}_{k}$ to indicate that some (hence all) elements of $\mathcal{E}_{j}$ embed into some (hence all) elements of $\mathcal{E}_{k}$. For each class $\mathcal{E}_{k}$, pick one representative $T\left[x_{i_{k}}\right]$, and apply Corollary 3.6 to $\left\{T\left[x_{i_{k}}\right]: k \in \omega\right\}$. (We do not need this procedure of picking elements to be computable since we only use it to obtain a finite amount of information detailed below.) This gives us a $K$ such that for all $k \geq K$, there are infinitely many $j>k$ such that $\mathcal{E}_{k} \hookrightarrow \mathcal{E}_{j}$.

Consider the equivalence classes $\mathcal{E}_{0}, \ldots, \mathcal{E}_{K-1}$. Divide these classes and renumber them so that $\mathcal{E}_{0}, \ldots, \mathcal{E}_{q}$ are the finite ones. Then, just as above in Case 1 , the set

$$
X=\left\{x \in F: T[x] \in \mathcal{E}_{0} \cup \cdots \cup \mathcal{E}_{q}\right\}
$$

is finite. Therefore, $G=F-X$ is a $\Delta_{2}^{0}$ set to which we can apply Lemma 3.17. Therefore, $T$ is not computably categorical.

Oddly, the remaining case turns out to be the hardest. This is the situation in which we have infinitely many isomorphism types appearing above $r$, all of finite type, with every 
such isomorphism type embedding into every other one. One would think that with so many embeddings at hand the proof would be easy. Alternatively, Lemma 2.10 shows that infinitely many of these types must fail to be of strongly finite type, hence must have embeddings available within them to satisfy all the requirements. Curiously, the presence of so many types and embeddings interferes with the availability of non-strongly-finite types, and vice versa, so that in the end we must use a completely different approach. The following lemma will not be used directly in the proof of Proposition 3.1, but it is necessary to finish the proof of Case 1 in Lemma 3.16.

Lemma 3.19 Let $T$ be a tree of finite height with root $r$, such that every successor tree above $r$ is of finite type. Suppose there is an infinite set $X=\left\{x_{0}, x_{1}, \ldots\right\}$ of immediate successors of $r$ satisfying:

1. $X$ is $\Delta_{2}^{0}$; and

2. For all $x_{i}, x_{j} \in X, T\left[x_{i}\right] \equiv T\left[x_{j}\right]$; and

3. $\left\{T\left[x_{i}\right]\right\}$ includes infinitely many distinct isomorphism types.

Then $T$ is not computably categorical.

Proof. To simplify the proof, we will assume that $X$ contains every immediate successor of $r$ in $T$. The finite-injury construction we present can readily be adapted to the more general case, using $\Delta_{2}^{0}$-approximations to $X$. We begin by presenting the particular case in which ht $(T)=4$. As no tree of height $<4$ satisfies the hypotheses of the lemma, this will serve as the base case for an induction on the height of $T$. Suppose ht $(T)=4$ and every successor tree above $r$ embeds into every other such successor tree, and there are infinitely many isomorphism types occurring among these successor trees and they are all of finite type. Each time a node becomes established at level 1 in $T$, we know that it is the root of a successor tree, so we define it to be the next $x_{i}$. Since the successor trees all embed into each other, they must all have the same height - namely 3 , since $\mathrm{ht}(T)=4$ - so every node at level 1 of $T$ eventually is identified as $x_{i}$ for some $i$. Also, it is not hard to see that since each $T\left[x_{i}\right]$ has finite type, each $T\left[x_{i}\right]$ must be $\omega$-branching at its root for the conditions of the lemma to hold.

If $y$ is an immediate successor of any $x_{i}$, then the isomorphism type of $T[y]$ is determined by the number of immediate successors $y$ has. By Lemma 3.9, since $T\left[x_{i}\right] \equiv T\left[x_{j}\right]$ are finite type trees, they have exactly the same infinitely occurring successor trees. Therefore, there is a finite list $n_{1}<n_{2}<\cdots<n_{k}$ such that $n_{j} \leq \omega$ for each $1 \leq j \leq k$, and for every $i$, the successor trees in $T\left[x_{i}\right]$ which occur infinitely often are the $n_{j}$-branching trees of height 2 for $1 \leq j \leq k$. We next show that $k=1$. Suppose that $k>1$. Under this assumption, $T\left[x_{i}\right]$ has an infinitely occurring successor tree which embeds into a nonisomorphic infinitely occurring successor tree. Since this violates the definition of finite type, we must have $k=1$. Let $\gamma=n_{1}$ be such that the unique infinitely occurring successor tree in every $T\left[x_{i}\right]$ is $\gamma$-branching.

Furthermore, we claim that for any $m$ such that $\gamma<m \leq \omega$, each $T\left[x_{i}\right]$ has exactly the same number of $m$-branching successor trees. To see this fact, first suppose $\gamma<\omega$, 
$T\left[x_{i}\right]$ has $u_{i}$ many $\omega$-branching trees, $T\left[x_{j}\right]$ has $u_{j}$ many $\omega$-branching trees and $u_{i}<u_{j}$. We know $T\left[x_{j}\right] \hookrightarrow T\left[x_{i}\right]$, so each $\omega$-branching successor tree in $T\left[x_{j}\right]$ must map into an $\omega$-branching successor tree in $T\left[x_{i}\right]$, and because of the heights of the trees, two different $\omega$-branching successor trees in $T\left[x_{j}\right]$ cannot map into the same $\omega$-branching successor tree in $T\left[x_{i}\right]$. Therefore, we have an immediate contradiction. Second, fix the maximal $m<\omega$ such that $\gamma<m$ and $T\left[x_{i}\right]$ has $v_{i}$ many $m$-branching successor trees for some $v_{i}>0$. (Because $T\left[x_{i}\right]$ has finite type, it has only finitely many different isomorphism types among its successor trees. Therefore, either there is no $m$ with $\gamma<m<\omega$ such that $T\left[x_{i}\right]$ has an $m$-branching successor tree, in which case we have established our claim, or there is a maximal such $m$.) Each of these trees must map into a successor tree in $T\left[x_{j}\right]$ which is at least $m$-branching (but not $\omega$-branching since those successor trees are already mapped to by the $\omega$-branching successor trees in $T\left[x_{i}\right]$ ) and no two such $m$-branching successor tree in $T\left[x_{i}\right]$ can map into the same successor tree in $T\left[x_{j}\right]$. Therefore, $T\left[x_{j}\right]$ must have at least $v_{i}$ many successor trees which are at least $m$-branching but not $\omega$-branching. However, if $T\left[x_{j}\right]$ has a successor tree that is more than $m$-branching but less than $\omega$-branching, then it has no place to map to under $T\left[x_{j}\right] \hookrightarrow T\left[x_{i}\right]$. Therefore, $T\left[x_{j}\right]$ has at least $v_{i}$ many successor trees which are exactly $m$-branching. By switching the roles of $T\left[x_{i}\right]$ and $T\left[x_{j}\right]$, we see that $T\left[x_{j}\right]$ must have exactly $v_{i}$ many successor trees which are $m$-branching. We can obviously continue this process with the next largest number which is less than $m$, greater than $\gamma$ and such that $T\left[x_{i}\right]$ has at least one successor tree with that number of branches.

We now know that any $T\left[x_{i}\right]$ and $T\left[x_{j}\right]$ must look identical with respect to their successor trees which are more than $\gamma$-branching. However, there must be infinitely many different isomorphism types among the $T\left[x_{i}\right]$ trees. These differences in isomorphism type must be due to the successor trees which are less than $\gamma$-branching. Therefore, infinitely many $T\left[x_{i}\right]$ contain a node $w$ at level 1 in $T\left[x_{i}\right]$ which has fewer than $\gamma$ immediate successors. We will use as our witness nodes those nodes $w$ with $<\gamma$ immediate successors, with at most one witness node in each successor tree. When necessary to diagonalize, we add more successors to $\varphi_{e}(w)$ in $T^{\prime}$ so that it has exactly $\gamma$ successors.

We identify the witness nodes as follows. At any given stage, the witness node in $T\left[x_{i}\right]$ should be that node $x \in T\left[x_{i}\right]$ with $<\gamma$ successors which has level $T_{s}(x)=2$ and which has gone the longest without acquiring any new successors. That is, for each $x$ at level 1 in $T\left[x_{i}\right]$, let

$$
\left.t_{x}=(\mu t \geq x) \text { [all successors of } x \text { in } T_{s} \text { are in } T_{t}\right],
$$

and choose as the witness node in $T\left[x_{i}\right]$ at stage $s$ the smallest $x$ such that $t_{x}$ is minimal.

However, it is possible that $T\left[x_{i}\right]$ contains no nodes with $<\gamma$ successors, so we must search among different successor trees. At first, we choose $w_{0, s}$ to be the witness node in $T\left[x_{0}\right]$. If this witness node changes at some subsequent stage $s_{1}$, then we choose $w_{0, s_{1}+1}$ to be the witness node in $T\left[x_{1}\right]$. If at a subsequent stage $s_{2}$ the witness node in $T\left[x_{1}\right]$ changes, then we change $w_{0, s_{2}+1}$ back to the (current) witness node in $T\left[x_{0}\right]$, then $T\left[x_{1}\right]$ again, then $T\left[x_{2}\right]$, then back to $T\left[x_{0}\right]$, and so on. In general, let $s_{k}$ be the next stage (if any) after $s_{k-1}$ at which $w_{0, s}$ changes, and choose $w_{0,1+s_{k}}$ to be the witness node in $T\left[x_{i}\right]$ at stage $1+s_{k}$, where $k=\langle i, j\rangle$. 
The properties proved above guarantee that there must be infinitely many $T\left[x_{i}\right]$ containing nodes $x$ such that $x$ is an immediate successor of $x_{i}$ and such that $x$ has $<\gamma$ successors, so eventually $w_{0, s}$ converges to some $w_{0}$. At the same time, we do the same for the witness node $w_{1, s}$ for $\mathcal{R}_{1}$, looking only at witness nodes in successor trees $T\left[x_{j}\right]$ in which $w_{0, s}$ has never yet been located, and so on by a standard finite-injury process.

Sublemma 3.20 For every e, $w_{e}=\lim _{s} w_{e, s}$ exists and has $<\gamma$ successors in $T$.

Proof. Assume by induction that the lemma holds for all $i<e$. Then each of $w_{0}, \ldots w_{e-1}$ lies above one of $x_{0}, \ldots x_{k}$, for some $k$. By our assumptions about $T$, there must be a node $y$ in some $T\left[x_{j}\right]$ with $j>k$ such that $\operatorname{level}_{T}(y)=2$ and $y$ has $<\gamma$ successors. Assume that this $y$ is chosen to have minimal $t_{y}$ among all such nodes in $T\left[x_{j}\right]$. (Hence $y$ acquires no new successors after stage $t_{y}$. If there is more than one $y$ with minimal $t_{y}$, we take $y$ to be the smallest of them.)

Pick a stage $s_{0}$ by which $x_{0}, \ldots x_{j}$ are all established, so that $T\left[x_{j}\right]$ will be available to us when we define $w_{e, s}$ at all $s \geq s_{0}$. (Hence $w_{e, s}$ will never be undefined after $s_{0}$.) If $w_{e, s}$ fails to converge to a limit, then it must be in $T\left[x_{j}\right]$ at infinitely many stages $s$, according to our instructions for choosing $w_{e, s}$. Since $t_{y}$ is minimal, we must have $w_{e, s}=y$ at cofinitely many of the stages such that $w_{e, s} \in T\left[x_{j}\right]$. Hence $w_{e, s}=y$ at some stage $s>t_{y}$. But then $w_{e, s}=y$ for all subsequent stages $s$ as well, proving the sublemma. (Possibly $w_{e, s}$ converged to some other limit in some other $T\left[x_{k}\right]$ instead of converging to $y$, of course.) However, in any case, the construction guarantees that the limit must have $<\gamma$ successors in $T$.

We build $T^{\prime}$ by copying $T$ at each stage $s$, with the following provision. Find each $e \leq s$ such that $w_{e, s}$ is defined and $\varphi_{e, s}\left(w_{e, s}\right) \downarrow$ (say $y=\varphi_{e, s}\left(w_{e, s}\right)$ ) and $f_{s}^{-1}(y)$ lies at level 2 in $T_{s}$ and has fewer than $\gamma$ successors in $T_{s}$. If there is no such $e$, simply extend $f_{s}$ to $f_{s+1}$ by adding new elements to $T_{s+1}^{\prime}$. If there is, then for the least such $e$, add new elements to $T^{\prime}$ so that $y$ has exactly $\gamma$ successors in $T^{\prime}$. We also add new elements to $T^{\prime}$ to be the image of $T_{s}\left[f_{s}^{-1}(y)\right]$ under $f_{s+1}$. The elements of $T_{s+1}^{\prime}[y]$ will not lie in the image of the limit $f$, but $T$ and $T^{\prime}$ will still be isomorphic, since every $x_{i}$ has infinitely many immediate successors with exactly $\gamma$ successors of their own. Notice that as in previous constructions with auxiliary trees, if a node $y \in T^{\prime}$ is removed from the range of $f$, then it permanently becomes part of an auxiliary $\gamma$-branching subtree of $T^{\prime}$. We have simply added one more such node above $f\left(x_{i}\right)$ in $T^{\prime}$ during this redefinition of $f$. The only injuries to $\mathcal{R}_{e}$ occur when $w_{i, s+1} \neq w_{i, s}$ for some $i<e$. Thus we have ensured that $y=\varphi_{e, s}\left(w_{e, s}\right)$ has $\gamma$ successors in $T^{\prime}$, while $w_{e, s}$ has $<\gamma$ successors in $T_{s}$. If $w_{e, s}=w_{e}$, then $w_{e, s}$ acquired no new successors in $T$ after stage $s$, leaving $\mathcal{R}_{e}$ satisfied.

Two minor modifications to this strategy are required for $\mathcal{R}$ and $\mathcal{N}$ strategies to work together. First, as we have done before, we assign $e+1$ many witnesses to $\mathcal{R}_{e}$ and we check whether $f_{s}^{-1}(y) \prec u$ for $u<e$ before allowing $\mathcal{R}_{e}$ to act. This modification insures that $f=\lim _{s} f_{s}$ exists and that $T \cong T^{\prime}$. Second, since there are parts of $T_{s}^{\prime}$ which are not in the range of $f_{s}$, it is possible that $y$ lies at level 2 in $T_{s}^{\prime}$ but it is not in the range of $f_{s}$. In this 
case, $y$ already has $\gamma$ successors because of the action of some $\mathcal{R}$ requirement. Therefore, if $w_{e, s}=w_{e}$, then $\mathcal{R}_{e}$ has already won without needing to act.

We now assume by induction that for all trees $T$ with $h t(T)<n$ satisfying the hypotheses of the lemma, we have a construction of a tree $T^{\prime}$ which is isomorphic to $T$ but not computably isomorphic to it. Let $\operatorname{ht}(T)=n$, and suppose that every successor tree above $r$ embeds into every other such successor tree, and that there are infinitely many isomorphism types occurring among these successor trees and that they are all of finite type. Each time a node becomes established at level 1 in $T$, we know that it is the root of a successor tree, so we define it to be the next $x_{i}$. Since the successor trees all embed into each other, they must all have the same height - namely $n-1$, since $\mathrm{ht}(T)=n-$ so every node at level 1 of $T$ eventually is identified as $x_{i}$ for some $i$.

Notice that for $x_{i}$ and $x_{j}$ at level 1 in $T$, we have that $T\left[x_{i}\right] \equiv T\left[x_{j}\right]$ and that both of these trees are of finite type. Therefore, by Lemma 3.9, the set of isomorphism types which occur infinitely often among the successor trees of $x_{i}$ in $T\left[x_{i}\right]$ is exactly the same as the set of isomorphism types which occur infinitely often among the successor trees of $x_{j}$ in $T\left[x_{j}\right]$. Let these types be $I_{1}, \ldots I_{p}$. We will consider two cases.

Case 1. Suppose there are infinitely many $i$ such that some finite-appearing successor tree in $T\left[x_{i}\right]$ embeds into one of $I_{1}, \ldots I_{p}$. Then (without loss of generality) there must be infinitely many $i$ such that some finite-appearing successor tree in $T\left[x_{i}\right]$ embeds into $I_{1}$. The construction in this case will be much the same as the construction in the case where ht $(T)=4$. The witness nodes will be roots of finite-appearing successor trees in various $T\left[x_{i}\right]$, and we will embed those finite-appearing trees into successor trees in $T\left[x_{i}\right]$ isomorphic to $I_{1}$ when necessary to satisfy the requirements. In this general case, however, it is more difficult to locate the witness nodes.

Since $I_{1}$ has strongly finite type, we can use finitely much information to construct a nice copy of $I_{1}$. By a nice copy, we mean both that we know the isomorphism type of every subtree of the form $I_{1}[a]$ and also that $x \prec y$ implies that $x<y$. We use this copy of $I_{1}$, along with the notion of a basic embedding, to pick out witness nodes in $T$.

Definition 3.21 Two nodes $x$ and $y$ in a tree $S$ are siblings if they have the same immediate predecessor in $S$. (This includes the case $x=y$.)

Definition 3.22 An embedding $\psi: S \hookrightarrow T$ is basic if it maps the root of $S$ to the root of $T$ and for every pair of siblings $y_{0}<y_{1}$ in $T$, if $T\left[y_{0}\right] \cong T\left[y_{1}\right]$ and $y_{1} \in \operatorname{range}(\psi)$, then also $y_{0} \in$ range $(\psi)$ and $\psi^{-1}\left(y_{0}\right)<\psi^{-1}\left(y_{1}\right)$. (Here, of course, $<$ refers to the standard ordering on $\omega$, not to the tree structure of $T$ or $S$.)

The intuition for building a (not necessarily computable) basic embedding is that, having mapped $x$ to $\psi(x)$, we consider the immediate successors $x_{0}, x_{1}, \ldots$ of $x$ in numerical order (so $x_{i}<x_{i+1}$ for all $i$ ). Having defined $\psi$ on $x_{0}, \ldots x_{i}$, we choose an isomorphism type above $\psi(x)$ into which to map $S\left[x_{i+1}\right]$, and let $\psi\left(x_{i+1}\right)$ be the least root $y$ of a successor tree of that isomorphism type above $\psi(x)$ such that $y$ is not already in the range of $\psi$. The only problem 
with this algorithm is that several successor trees from $S$ may have to map into the same finitely occurring successor tree in $T$. We show how to handle this problem below. Notice that for our nice copy of $I_{1}$, it is computable for finite trees $S$, uniformly in $S$, whether a basic embedding of $S$ into $I_{1}$ exists, and also whether any specific map $\psi: S \rightarrow I_{1}$ is a basic embedding or not.

We prove the following sublemmas about basic embeddings. Although they apply to any trees of strongly finite type, we will apply them to our nice copy of $I_{1}$.

Sublemma 3.23 Let $S$ be a tree with finite type and $U$ be a tree with strongly finite type. If there is an embedding $f: S \hookrightarrow U$, then there is a basic embedding $g: S \hookrightarrow U$.

Proof. We proceed by induction on the height of $U$. If $U$ has height 1 , then $S$ must have height 1 and they both consist only of a root. The basic embedding $g$ sends the root of $S$ to the root of $U$. Assume $U$ has height greater than 1 and that we know the theorem by induction for all trees of lower heights.

Fix the embedding $f$ and define $g$ to send the root of $S$ to the root of $U$. We describe how $g$ behaves on all successor trees $S[x]$ of the root in $S$ by splitting into two cases. Let $y_{0}, y_{1}, \ldots$ be all the nodes at level 1 in $U$ numbered so that $i<j$ implies $y_{i}<y_{j}$. Assume that the successor trees $U\left[y_{i}\right]$ for $i \leq n$ are exactly the successor trees whose isomorphism types occur only finitely often in $U$. (The proof below does not depend on the fact that the finitely occurring successor trees have roots which are less than the roots of the infinitely occurring successor trees. It does, however, simplify the notation.) We first consider those successor trees of $S$ that $f$ embeds into some $U\left[y_{i}\right]$ for $i>n$ and second we consider those successor trees of $S$ which $f$ embeds into some $y_{i}$ for $i \leq n$.

Let $x_{i_{0}}<x_{i_{1}}<\cdots$ be the nodes at level 1 in $S$ such that $f$ embeds $S\left[x_{i_{k}}\right]$ into one of the infinitely occurring isomorphism types of successor trees in $U$. Fix $j_{k}$ to be the index of the node $y_{j_{k}}$ at level 1 in $U$ such that $f: S\left[x_{i_{k}}\right] \hookrightarrow U\left[y_{j_{k}}\right]$. Define $g$ on the trees $S\left[x_{i_{k}}\right]$ by recursion on $k$. Let $g\left(x_{i_{k}}\right)=y$ where $y$ is the $\leq_{\mathbb{N}}$-least node at level 1 in $U$ such that $U[y] \cong U\left[y_{j_{k}}\right]$ and $y$ is not in the range of $g$ yet. Since $S\left[x_{i_{k}}\right] \hookrightarrow U[y]$, by induction there is a basic embedding of these trees. Let $g$ be defined on $S\left[x_{i_{k}}\right]$ to be such an embedding.

Next, consider the successor trees $S[x]$ in $S$ such that $f$ maps $S[x]$ into one of the finitely occurring isomorphism types of successor trees in $U$. Notice that if $f$ maps two successor trees $S\left[x_{1}\right]$ and $S\left[x_{2}\right]$ into the same successor tree $U[y]$, then $y$ is not in the range of $f$. We consider each of the finitely occurring isomorphism types separately. Fix one of these types and assume without loss of generality that $U\left[y_{0}\right], \ldots, U\left[y_{m}\right]$ are the successor trees with this isomorphism type and that $y_{0}<\cdots<y_{m}$. For $i \leq m$, let $Y_{i}$ be the set of nodes $x$ at level 1 in $S$ such that $f$ maps $S[x]$ into $S\left[y_{i}\right]$. We consider first the sets $Y_{i}$ which have size 1 and then the sets which have size at least 2. (Of course, it is possible that some $Y_{i}$ are empty and we ignore these sets.)

For each $Y_{i}$ with size 1 , fix the unique successor tree in $S$ which maps into $U\left[y_{i}\right]$. Let $x_{i_{0}}<\cdots<x_{i_{k}}$ be the root nodes of these successor trees. Define $g\left(x_{i_{l}}\right)=y_{l}$ for $l \leq k$. Since we know $S\left[x_{i_{l}}\right] \hookrightarrow U\left[y_{l}\right]$, we can extend our definition of $g$ (by the induction hypothesis) to be a basic embedding between these subtrees. 
We consider the remaining $Y_{i}$ with size at least 2 individually. Fix such a $Y_{i}$ and let $x_{0}, x_{1}, \ldots \in Y_{i}$ be the nodes of level 1 in $S$ such that $f$ embeds $S\left[x_{k}\right]$ into $U\left[y_{i}\right]$. (This list may be either finite or infinite.) Consider an auxiliary tree $S_{i}$ formed by taking a root node and attaching the trees $S\left[x_{k}\right]$ immediately above the root. Let $u \leq m$ be the least index such that we have not defined $g$ mapping into $U\left[y_{u}\right]$ yet. By our assumptions, we know that $S_{i}$ embeds in $U\left[y_{u}\right]$. (Notice that this is where we use the fact that $Y_{i}$ has size at least 2. In this case, the node $y_{i}$ was not in the image of $f$ because $f$ mapped more than one successor tree into $\left.U\left[y_{i}\right].\right)$ By the induction hypothesis, there is a basic embedding of $S_{i}$ into $U\left[y_{u}\right]$. Let $g$ be the restriction of such a basic embedding to all nodes in $S_{i}$ except the root node. This definition of $g$ maps all of the $S\left[x_{k}\right]$ trees into $U$ in a basic way. (That is, any violation of the requirement on the images of siblings in the restricted version of $g$ would have been a violation of the restriction on the basicness of the embedding of $S_{i}$.) Performing the action of the last two paragraphs for each isomorphism type of a finitely occurring successor tree in $U$ completes the description of $g$.

Sublemma 3.24 Let $U$ be a finite height tree of strongly finite type and let $f: U \hookrightarrow U$. Then for all $x$ at level 1 , we have that $f(x)$ is at level 1 and $U[x] \cong U[f(x)]$. (We are not claiming that $f$ is an isomorphism, which it need not be, but only that these successor trees are isomorphic.)

Proof. Fix a node $x$ at level 1 in $U$ such that $f(x) \neq x$. We split into the cases when $U[x]$ is a finitely occurring isomorphism type and when $U[x]$ is an infinitely occurring isomorphism type.

Suppose $U[x]$ is a finitely occurring isomorphism type and there is some $m<n$ such that $f^{m}(x)=f^{n}(x)$. Now $f$, being an embedding, has a one-to-one inverse $g$, with $\operatorname{dom}(g)=$ range $(f)$. So $f^{n-m}(x)=g^{m}\left(f^{n}(x)\right)=g^{m}\left(f^{m}(x)\right)=x$, forcing

$$
U[x] \hookrightarrow U[f(x)] \hookrightarrow U\left[f^{n-m}(x)\right]=U[x] .
$$

Since these are strongly finite trees, $U[x] \cong U[f(x)]$ by Lemma 2.10, and moreover, $1 \leq$ $\operatorname{level}(f(x)) \leq \operatorname{level}\left(f^{n-m}(x)\right)=1$.

Now suppose $U[x]$ is a finitely occurring isomorphism type but there is no $m<n$ such that $f^{n}(x)=f^{m}(x)$. We first show that there must be a $n_{1}$ such that level $\left(f^{n_{1}}(x)\right)>1$. Because $U[x]$ is a finitely occurring isomorphism type and $U$ has strongly finite type, if $U[x] \hookrightarrow U[y]$ and level $(y)=1$, then $U[y]$ is a finitely occurring successor tree. Fix $y$ such that level $(y)=1$ and $f$ embeds $U[x]$ into $U[y]$. There are two possibilities, either $y \prec f(x)$ (in which case level $(f(x))>1$ and we are done) or $y=f(x)$ (in which case level $(f(x))=1$ ). If $f(x)=y$, then we repeat the above process to gain information about $f^{2}(x)$. Since $U[y]=U[f(x)]$ is a finitely occurring successor tree, $f$ must embed $U[f(x)]$ into a finitely occurring successor tree $U[z]$ with level $(z)=1$. Again, either we have $z \prec f^{2}(x)$ (in which case we are done) or $z=f^{2}(x)$. In the latter case, we repeat the process again. Each time we repeat this process, we either find that level $\left(f^{n}(x)\right)>1$ (and we are finished) or $f^{n}(x)$ is the root of another finitely occurring successor tree. Since $f^{n}(x) \neq f^{m}(x)$ for all $n \neq m$, we can never repeat the 
root of a particular finitely occurring successor tree during this process. Because there are only finitely many finitely occurring successor trees, this process must stop at some value $n_{1}$ with level $\left(f^{n_{1}}(x)\right)>1$.

Let $x_{1}$ be the node at level 1 such that $x_{1} \prec f^{n_{1}}(x)$. We next show that it is not the case that $f^{k}\left(x_{1}\right)=x_{1}$ for some $k$. For a contradiction, assume that $f^{k}\left(x_{1}\right)=x_{1}$ for some $k$. Then, because $f^{p+k}\left(x_{1}\right)=f^{p}\left(x_{1}\right)$ for all $p$, there must be a node $y_{1}$ such that $f^{n_{1}}\left(y_{1}\right)=x_{1}$. However, then we have $f^{n_{1}}\left(y_{1}\right)=x_{1} \prec f^{n_{1}}(x)$, so $y_{1} \prec x$ which implies that $y_{1}$ is the root. Since $f$ must take the root to the root, this gives the desired contradiction.

Repeating the argument above for $x_{1}$, there must be an $n_{2}$ such that $\operatorname{level}\left(f^{n_{2}}\left(x_{1}\right)\right)>1$. Therefore,

$$
\operatorname{level}\left(f^{n_{1}+n_{2}}(x)\right)>\operatorname{level}\left(f^{n_{2}}\left(x_{1}\right)\right)>1
$$

and hence level $\left(f^{n_{1}+n_{2}}(x)\right)>2$. If we now let $x_{2}$ be the node of level 1 such that $x_{2} \prec$ $f^{n_{2}}\left(x_{1}\right)$, we can repeat the argument to show there is an $n_{3} \operatorname{such}$ that level $\left(f^{n_{1}+n_{2}+n_{3}}(x)\right)>3$. Repeating this process contradicts the fact that $U$ has finite height. Therefore, it cannot be the case that $f^{k}(x) \neq x$ for all $k$. We have now shown that $f$ must permute the successor trees $U[x]$ which have finitely occurring isomorphism types.

It remains to consider $x$ such that $U[x]$ has an infinitely occurring isomorphism type. By the argument above, $f$ cannot map $U[x]$ into a finitely occurring isomorphism type because it must permute those types. Therefore, $f$ must embed $U[x]$ into some $U[z]$ which has an infinitely occurring isomorphism type. However, this means that $U[x] \cong U[z]$ since $U$ has strongly finite type and hence we must have $f(x)=z$.

Sublemma 3.25 If $T_{1} \cong T_{2}$ have strongly finite type and $f: T_{1} \hookrightarrow T_{2}$, then for all $x$ at level 1 in $T_{1}, f(x)$ is at level 1 in $T_{2}$ and $T_{1}[x] \cong T_{2}[f(x)]$.

Proof. Let $g$ be any isomorphism from $T_{2}$ to $T_{1}$. This sublemma follows from Sublemma 3.24 by considering $g f: T_{1} \hookrightarrow T_{1}$. Notice that as in the proof of Sublemma 3.24, if $T_{1}[x]$ has finitely occurring isomorphism type, then $T_{2}[f(x)]$ has finitely occurring isomorphism type. Furthermore, if $y$ is at level 1 in $T_{2}$ and $T_{2}[y]$ has finitely occurring isomorphism type, then $y$ is in the range of $f$.

Sublemma 3.26 Let $U$ be a finite height tree of strongly finite type, $f: U \hookrightarrow U$, and $k<h t(U)$. For all nodes $x$ at level $k, f(x)$ has level $k$ and $U[x]$ is isomorphic to $U[f(x)]$.

Proof. This follows by induction on $k$ using Sublemma 3.24.

Sublemma 3.27 Let $T_{1} \cong T_{2}$ be finite height trees of strongly finite type. There is exactly one basic embedding $f: T_{1} \hookrightarrow T_{2}$ and $f$ is an isomorphism.

Proof. We proceed by induction on the height of $T_{1}$. The case for height 1 is trivial. Assume the sublemma holds for all trees of height less than the height of $T_{1}$. By Sublemma 3.23, we know that there is a basic embedding $f: T_{1} \hookrightarrow T_{2}$. We need to show that $f$ is onto (and therefore is an isomorphism) and is unique. 
We know that $f$ sends the root of $T_{1}$ to the root of $T_{2}$. Consider any node $x$ at level 1 in $T_{2}$. We have already seen that if $x$ has an infinitely occurring isomorphism type then $x$ cannot be equal to $f(y)$ where $T_{1}[y]$ has finitely occurring type. Also, if $x$ is not in the range of $f$, then we have an immediate contradiction since some $z>x$ with $T_{2}[z] \cong T_{2}[x]$ must be in the range of $f$ because $T_{1}$ has infinitely many nodes $u$ at level 1 with $T_{1}[u] \cong T_{2}[x]$. This contradicts the fact that $f$ is basic. Therefore, $x$ must be in the range of $f$. Furthermore, if $x$ is the $k$-th node at level 1 in $T_{2}$ with its isomorphism type (where we measure $k$-th using the $\leq_{\mathbb{N}}$-ordering), it must be the case that $f(u)=x$ where $u$ is the $k$-th node in $T_{1}$ with this type. Therefore, for nodes at level 1 in $T_{1}$ with infinitely occurring isomorphism types, the map $f$ is uniquely determined. By induction, the values of $f$ above these nodes are uniquely determined and give an isomorphism between the successor trees.

If $T_{2}[x]$ is a finitely occurring type, then we already know that $x=f(y)$ for some $y \in T_{1}$. By an argument similar to the one above, the value of $y$ is uniquely determined, and by the induction hypothesis, $f$ is a uniquely determined isomorphism from $T_{1}[y]$ to $T_{2}[x]$.

Sublemma 3.28 For each basic embedding $\psi: S \hookrightarrow I_{1}$, the restriction of $\psi$ to $S \cap\{r, 0, \ldots s\}$ is also a basic embedding. (Here $r$ is the root of $S$.)

This is clear from the definition of basic embedding.

Sublemma 3.29 Let $S$ and $I_{1}$ be finite height trees such that $S$ is of finite type and $I_{1}$ is of strongly finite type. Suppose every basic embedding $S \hookrightarrow I_{1}$ includes the node $y$ of $I_{1}$ in its image. Then there is an such that every basic embedding of $S \cap\{r, 0,1, \ldots, s\}$ into $I_{1}$ includes $y$ in its image.

It then follows from Sublemma 3.28 that every basic embedding of every $S \cap\{0,1, \ldots, t\}$ into $I_{1}$ with $t \geq s$ includes $y$ in its image.

Proof. Our argument is purely classical and we do not claim (or need) any effectiveness in this sublemma. We proceed by induction on the height of $I_{1}$. The case when $I_{1}$ has height 1 (and hence consists of only the root) is trivial. Assume that the height of $I_{1}$ is greater than 1 and that the sublemma holds for all trees of shorter height. We split the argument into two cases: when $y$ is contained in one of the finitely occurring successor trees in $I_{1}$ and when $y$ is contained in one of the infinitely occurring successor trees in $I_{1}$. It suffices to show that if $y$ is contained in the range of all basic embeddings, then there is a finite subtree $U$ of $S$ for which all basic embeddings of $U$ into $I_{1}$ hit $y$.

First, consider the case when $y$ is contained in one of the finitely occurring successor trees of $I_{1}$. Let $S_{1}$ be the subtree of $S$ consisting of the root plus all the successor trees which do not embed into any infinitely occurring successor tree in $I_{1}$. Let $J_{1}$ be the subtree of $I_{1}$ containing the root and all the finitely occurring successor trees in $I_{1}$. We denote the successor trees in $J_{1}$ by $J_{1}\left[z_{0}\right], \ldots, J_{1}\left[z_{l}\right]$. Notice that not only does $S_{1} \hookrightarrow J_{1}$, but there is a basic embedding $S \hookrightarrow I_{1}$ such that $S \backslash S_{1}$ is mapped into $I_{1} \backslash J_{1}$. This basic embedding can be obtained by 
fixing a basic embedding $S_{1} \hookrightarrow J_{1}$ and then mapping each successor tree $S[x]$ not in $S_{1}$ (by induction on $x$ ) by a basic embedding into $I_{1}[z]$ where $z$ is the <-least node at level 1 in $I_{1} \backslash J_{1}$ which has not yet been mapped into.

We denote the finitely occurring successor trees in $S_{1}$ by $S_{1}\left[x_{0}\right], \ldots, S_{1}\left[x_{k}\right]$ with the assumption that $x_{0}<\cdots<x_{k}$. For simplicity of notation, we assume that there is only one isomorphism type for an infinitely occurring successor tree in $S_{1}$ (the general case when there are finitely many such isomorphism types will be clear from the argument below) and we denote these successor trees $S_{1}\left[y_{0}\right], S_{1}\left[y_{1}\right], \ldots$ with the assumption that $y_{0}<y_{1}<\cdots$. We make no assumptions about the <-ordering between elements $x_{i}$ and $y_{j}$.

We claim that for any $z \in J_{1}$ at level 1 , either infinitely many copies of $S_{1}\left[y_{0}\right]$ can be embedded into $J_{1}[z]$ or else there is a finite upper bound $m_{z}$ on the number of copies of $S_{1}\left[y_{0}\right]$ that can be embedded into $J_{1}[z]$. To see this fact, consider an auxiliary tree $U$ formed by taking a root with infinitely many copies of $S_{1}\left[y_{0}\right]$ as successor trees. (Notice that since $S_{1}\left[y_{0}\right]$ has finite type, so does $U$.) Because $U$ has finite type, we know that it embeds into $J_{1}[z]$ if and only if all of its finite subtrees embed into $J_{1}[z]$. Therefore, if $U \hookrightarrow J_{1}[z]$, then we obtain a finite bound $m_{z}$ as above. Since there are only finitely many successor trees in $J_{1}$, we let $m^{\prime}$ be a finite number such that for all $z \in J_{1}$ at level 1 , if $m^{\prime}$ copies of $S_{1}\left[y_{0}\right]$ embed into $J_{1}[z]$, then infinitely many copies of $S_{1}\left[y_{0}\right]$ embed into $J_{1}[z]$. Finally, since there are $l+1$ many successor trees in $J_{1}$, then we let $m=m^{\prime}(l+1)$. The point of $m$ is that we know that if $m$ many copies of $S_{1}\left[y_{0}\right]$ are embedded into $J_{1}$, then at least $m^{\prime}$ many must have been embedded into some $J_{1}\left[z_{j}\right]$ and hence infinitely many copies of $S_{1}\left[y_{0}\right]$ could have been embedded into that successor tree.

We next define a finite tree $S_{1}^{\prime} \subset S_{1}$ whose basic embeddings into $J_{1}$ will encode (in a way made precise below) the possible successor trees $J_{1}[v]$ that a successor tree $S_{1}[u]$ could be sent to by a basic embedding of $S$ into $I_{1}$. $S_{1}^{\prime}$ consists of the root plus successor trees $S_{1}^{\prime}\left[x_{i}\right] \subset S_{1}\left[x_{i}\right]$ for $i \leq k$ and $S_{1}^{\prime}\left[y_{0}\right], \ldots, S_{1}^{\prime}\left[y_{m}\right]$. Each of the $S_{1}^{\prime}\left[y_{i}\right]$ trees are isomorphic with $S_{1}^{\prime}\left[y_{0}\right] \subset S_{1}\left[y_{0}\right]$. We pick these finite trees to have the following embedding properties, where $z$ ranges over all nodes at level 1 in $J_{1}$.

1. $S_{1}^{\prime}\left[x_{i}\right] \hookrightarrow I_{1}[v]$ for any $v \in I_{1} \backslash J_{1}$ (and the same for $S_{1}^{\prime}\left[y_{i}\right]$ ).

2. $S_{1}^{\prime}\left[x_{i}\right] \hookrightarrow J_{1}[z]$ if and only if $S_{1}\left[x_{i}\right] \hookrightarrow J_{1}[z]$ (and similarly for $S_{1}^{\prime}\left[y_{i}\right]$ ).

3. If $S_{1}\left[x_{i}\right] \hookrightarrow J_{1}[z]$ only by sending $x_{i}$ to $z$, then the same property holds for $S_{1}^{\prime}\left[x_{i}\right]$ (and similarly for $\left.S_{1}^{\prime}\left[y_{i}\right]\right)$.

4. Consider all possible choices of nonrepeating sequences $u_{0}, \ldots, u_{q}$ with each $u_{a}$ equal either to some $x_{i}$ (with $i \leq k$ ) or some $y_{j}$ (with $j \leq m$ ). Let $U$ denote the tree formed by taking a root and successor trees $S_{1}\left[u_{0}\right], \ldots, S_{1}\left[u_{q}\right]$ and let $U^{\prime}$ denote the finite tree formed by taking a root and successor trees $S_{1}^{\prime}\left[u_{0}\right], \ldots, S_{1}^{\prime}\left[u_{q}\right]$. Then, $U \hookrightarrow J_{1}[z]$ if and only if $U^{\prime} \hookrightarrow J_{1}[z]$.

The fact that we can pick finite subtrees with properties (1), (2) and (4) is clear from Lemma 2.9. To see that we can get property (3), suppose that all finite subtrees of $S_{1}\left[x_{i}\right]$ containing 
$x_{i}$ can be embedded into $J_{1}[z]$ without sending $x_{i}$ to $z$. Then for one of the finitely many isomorphism types of the successor trees in $J_{1}[z]$, arbitrarily large subtrees of $S_{1}\left[x_{i}\right]$ can be embedded into this type. But, then $S_{1}\left[x_{i}\right]$ can be embedded into a successor tree of $J_{1}[z]$ with this type, and hence there is an embedding of $S_{1}\left[x_{i}\right]$ into $J_{1}[z]$ which does not send $x_{i}$ to $z$.

Property (1) says that any embedding $S_{1}^{\prime} \hookrightarrow I_{1}$ must actually send $S_{1}^{\prime}$ into $J_{1}$. Properties (2) and (4) together say that any embedding $S_{1}^{\prime} \hookrightarrow J_{1}$ can be extended to an embedding $S_{1} \hookrightarrow J_{1}$ and hence to an embedding $S \hookrightarrow I_{1}$. Property (3) says that if this extension requires that a node at level 1 in $S_{1}$ map to a node at level 1 in $J_{1}$, then this requirement was already present for the embedding of $S_{1}^{\prime}$.

For any basic embeddings $f, g: S_{1}^{\prime} \hookrightarrow J_{1}$, we say $f \sim g$ if and only if the following two conditions hold.

$$
\begin{aligned}
& \forall i \leq k \forall j \leq l\left(\left(f\left(x_{i}\right) \in J_{1}\left[z_{j}\right] \leftrightarrow g\left(x_{i}\right) \in J_{1}\left[z_{j}\right]\right) \wedge\left(f\left(x_{i}\right)=z_{j} \leftrightarrow g\left(x_{i}\right)=z_{j}\right)\right) \\
& \forall i \leq m \forall j \leq l\left(\left(f\left(y_{i}\right) \in J_{1}\left[z_{j}\right] \leftrightarrow g\left(y_{i}\right) \in J_{1}\left[z_{j}\right]\right) \wedge\left(f\left(y_{i}\right)=z_{j} \leftrightarrow g\left(y_{i}\right)=z_{j}\right)\right)
\end{aligned}
$$

It is clear that $\sim$ is an equivalence relation and that up to $\sim$ equivalence, there are only finitely many basic embeddings $S_{1}^{\prime} \hookrightarrow J_{1}$. Let $f_{0}, \ldots, f_{q}$ be a list containing one element from each equivalence class. For a basic embedding $g: S \hookrightarrow I_{1}$, we say that $g \sim f_{i}$ if the restriction of $g$ to $S_{1}^{\prime}$ is equivalent to $f_{i}$.

Our definition of $m$ and the properties (1)-(4) above insure that for every basic embedding $g: S \hookrightarrow I_{1}$, there is an $f_{i}$ such that $g \sim f_{i}$ and that for each $f_{i}$, there is a basic embedding $g: S \hookrightarrow I_{1}$ such that $g \sim f_{i}$. It is in this sense that the embeddings $f_{0}, \ldots, f_{q}$ encode information about the possible basic embeddings of $S$ into $I_{1}$ when restricted to $S_{1}$.

We use the $f_{i}$ embeddings to prove the sublemma in the case when $y$ is a node in $J_{1}$. First, by the properties of the previous paragraph, a node $z_{j}$ at level $1 \mathrm{in} J_{1}$ is in the range of all basic embeddings $S \hookrightarrow I_{1}$ if and only if $z_{j}$ is in the range of $f_{i}$ for all $i \leq q$. Therefore, the finite tree $S_{1}^{\prime}$ is large enough to determine if the roots of the finitely occurring successor trees in $I_{1}$ are in the range of all basic embeddings.

Second, suppose that $y \in J_{1}\left[z_{j}\right]$, but $y \neq z_{j}$. For each $i \leq q$, we define a tree $U_{i}$ corresponding to $f_{i}$. Fix $i$ and consider all $u \in\left\{x_{0}, \ldots, x_{k}, y_{0}, \ldots, y_{m}\right\}$ such that $f_{i}$ maps $S_{1}^{\prime}[u]$ into $J_{1}\left[z_{j}\right]$. We split the definition of $U_{i}$ into two cases. If $f_{i}$ maps less than $m^{\prime}$ copies of trees of the form $S_{1}^{\prime}\left[y_{a}\right]$ into $J_{1}\left[z_{j}\right]$, then let $U_{i}$ consist of a root plus the successor trees $S_{1}[u]$ for which $f_{i}$ maps $S_{1}^{\prime}[u]$ into $J_{1}\left[z_{j}\right]$. If $f_{i}$ maps at least $m^{\prime}$ many $S_{1}^{\prime}\left[y_{a}\right]$ trees into $J_{1}\left[z_{j}\right]$, then let $U_{i}$ consist of a root plus the successor trees $S_{1}\left[x_{b}\right]$ for which $f_{i}$ maps $S_{1}^{\prime}\left[x_{b}\right]$ into $J_{1}\left[z_{j}\right]$ plus infinitely many successor trees isomorphic to $S_{1}\left[y_{0}\right]$. In either case, we know that $U_{i}$ embeds into $J_{1}\left[z_{j}\right]=I_{1}\left[z_{j}\right]$. Since the height of $I_{1}\left[z_{j}\right]$ is strictly less that the height of $I_{1}$, we can apply the induction hypothesis on height from the beginning of the sublemma. There is a finite subtree $U_{i}^{\prime}$ such that every basic embedding of $U_{i}^{\prime}$ into $I_{1}\left[z_{j}\right]$ has $y$ in its range. We expand each finite tree $S_{1}^{\prime}[u]$ to include the finite tree $U_{i}^{\prime} \cap S_{1}[u]$. Once we have expanded $S_{1}^{\prime}$ by performing this action for each $i \leq q$, we have that each basic embedding $S_{1}^{\prime} \hookrightarrow J_{1}$ must include $y$ in its range.

We now consider the case when $y$ is an element of one of the infinitely occurring successor 
trees in $I_{1}$. We begin by establishing facts about about when an infinitely occurring successor tree $I_{1}[x]$ is not in the range of every basic embedding $S \hookrightarrow I_{1}$. (Think of $x$ as the node at level 1 in $I_{1}$ such that $y \in I_{1}[x]$.) First, if there is a basic embedding which sends two successor trees of $S$ into $I_{1}[x]$, then $x$ is obviously not in the range of this embedding. Second, if there is some basic embedding $f$ such that $x$ is not in the range of $f$, then there is a basic embedding $g$ such that range $(g) \cap I_{1}[x]=\emptyset$. To see this second fact, let $x=x_{0}<x_{1}<\cdots$ be the nodes at level 1 in $I_{1}$ with $x_{i} \geq x$ and $I_{1}[x] \cong I_{1}\left[x_{i}\right]$. Since $f$ is basic and $x \notin \operatorname{range}(f)$, we know that for all $i, x_{i} \notin \operatorname{range}(f)$. Fix isomorphisms $h_{i}: I_{1}\left[x_{i}\right] \rightarrow I_{1}\left[x_{i+1}\right]$ which are also basic embeddings. (Such maps exist by Sublemma 3.27.) Define $g(u)$ as follows. If $f(u) \notin I_{1}\left[x_{i}\right]$ for any $i$, then $g(u)=f(u)$. If $f(u) \in I_{1}\left[x_{i}\right]$, then let $g(u)=h_{i}(f(u))$. Thus, $g$ shifts the image of $f$ on $I_{1}\left[x_{i}\right]$ to $I_{1}\left[x_{i+1}\right]$. Because $x_{i} \notin$ range $(f), g$ is a basic embedding.

From the previous paragraph, we see that if $x$ is the node at level 1 in $I_{1}$ such that $y \in I_{1}[x]$, then $x$ must be in the range of all basic embeddings and each basic embedding maps a single successor tree $S[u]$ into $I_{1}[x]$. We next show there is a bound on how big $u$ can be. For a contradiction, assume that there is no such bound. Let $J_{1}, \ldots, J_{m}$ be the isomorphism types of successor trees in $S$ that can be embedded into $I_{1}[x]$ by a basic embedding. Assume that $J_{1}, \ldots, J_{l}$ are types which occur finitely often as successor trees in $S$ and $J_{l+1}, \ldots, J_{m}$ are types which occur infinitely often. Because there is no bound on the $u$ such that $S[u]$ is embedded into $I_{1}[x]$ by a basic embedding, there must be basic embeddings which map all occurrences of the types $J_{1}, \ldots, J_{l}$ in $S$ as well as arbitrarily many copies of each of the types $J_{l+1}, \ldots, J_{m}$ into the successor trees $I_{1}[v]$ with $v<x$. However, as we saw above, if arbitrarily many finite copies of some $J_{i}$ can be embedded into some $I_{1}[v]$, then infinitely many copies can be embedded into $I_{1}[v]$. Therefore, there must be a basic embedding which sends all copies of the types $J_{1}, \ldots, J_{m}$ from $S$ into the successor trees $I_{1}[v]$ for $v<x$. This means that there is a basic embedding which does not map into $I_{1}[x]$ at all, contradicting our assumption that $y$ is in the range of all basic embeddings.

We conclude that if $I_{1}[x] \cap \operatorname{range}(f) \neq \emptyset$ for all basic $f$ (and hence $x \in \operatorname{range}(f)$ ), then there is a bound on the elements $u$ at level 1 such that $S[u]$ is mapped into $I_{1}[x]$ by a basic embedding. We can therefore limit the "level 1 width" of $S$ which we need to consider when looking at how much of $S$ is required to force the basic embeddings to intersect $I_{1}[x]$. This bound means that by an argument similar to the one when $y$ was assumed to be from a finitely occurring successor tree in $I_{1}$, we isolate a finite tree $U \subset S$ such that every basic embedding $U \hookrightarrow I_{1}$ must hit $x$. From here, we again consider the finitely many successor trees in $S$ which could map into $I_{1}[x]$ by a basic embedding and apply the inductive hypothesis on the height to handle the nodes $y \in I_{1}[x]$ with $y \neq x$.

We now begin our description of the construction for Case 1 of Lemma 3.19. The witness node in $T\left[x_{i}\right]$ at stage $s$ will be a node $z$ at level 1 in $T_{s}\left[x_{i}\right]$ such that $T_{s}[z] \hookrightarrow I_{1}$ but for which we believe $T[z] \nRightarrow I_{1}$. The first condition is easy to check, using our canonical copy of $I_{1}$. To satisfy the second condition, we want there to be a basic embedding of $T[z]$ into $I_{1}$ which is not surjective. Since $I_{1}$ is of strongly finite type, Sublemma 3.27 will then ensure that $T[z] \nRightarrow I_{1}$, and we will use this fact to diagonalize. 
At stage $s$, we define the witness node in $T_{s}\left[x_{i}\right]$ by finding the least pair $\langle z, y\rangle$ such that $z$ is at level 1 in $T_{s}\left[x_{i}\right]$ and there is a basic embedding of $T_{s}[z]$ into $I_{1}$ whose image does not contain $y$. We set $v_{i, s}=\langle z, y\rangle$, since it appears at this stage that $T[z]$ is the successor tree we want in $T\left[x_{i}\right]$, and define this $z$ to be the witness node in $T\left[x_{i}\right]$ at stage $s$.

Sublemma $3.30 \lim _{s} v_{i, s}$ converges if and only if there exists $z$ at level 1 in $T\left[x_{i}\right]$ such that $T[z]$ embeds into $I_{1}$ but is not isomorphic to $I_{1}$.

Proof. Assume $\lim _{s} v_{i, s}=v_{i}=\langle z, y\rangle$. Then $T[z]$ must embed into $I_{1}$, by Lemma 2.9. On the other hand, there must be a basic embedding of $T[z]$ into $I_{1}$ which omits $y$ from its image, by Sublemmas 3.23 and 3.29, and then Sublemma 3.27 ensures that $T[z] \nRightarrow I_{1}$.

Conversely, suppose that for some $z$ with level $_{T\left[x_{i}\right]}(z)=1$ we have $T[z] \hookrightarrow I_{1}$ but $T[z] \nsucceq I_{1}$. By Sublemma 3.23, there is a basic embedding of $T[z]$ into $I_{1}$, which cannot be surjective because $T[z] \nRightarrow I_{1}$. Thus there must be a least pair $\langle z, y\rangle$ such that some basic embedding of $T[z]$ into $I_{1}$ omits $y$. By Sublemma 3.28, we will have $v_{i, s} \leq\langle z, y\rangle$ for all sufficiently large s. Consider any pair $\left\langle z^{\prime}, y^{\prime}\right\rangle\left\langle\langle z, y\rangle\right.$. By our choice of $\langle z, y\rangle$, no basic embedding of $T\left[z^{\prime}\right]$ into $I_{1}$ omits $y^{\prime}$, so Sublemma 3.29 ensures that $v_{i, t} \neq\left\langle z^{\prime}, y^{\prime}\right\rangle$ for sufficiently large $t$. Thus $\lim _{s} v_{i, s}=\langle z, y\rangle$.

However, it is possible that $T\left[x_{i}\right]$ does not contain any finite-appearing successor tree which embeds into $I_{1}$, so we must search among different successor trees. At first, we choose $w_{0,0}$ to be the witness node in $T\left[x_{0}\right]$. If $v_{0, s_{1}} \neq v_{0,0}$ at some subsequent stage $s_{1}$, then we choose $w_{0, s_{1}}$ to be the witness node in $T\left[x_{1}\right]$ at stage $s_{1}$. If at a subsequent stage $s_{2}$ we have $v_{1, s_{2}} \neq v_{1, s_{1}}$, then we change $w_{0, s_{2}}$ back to the witness node in $T\left[x_{0}\right]$ at stage $s_{2}$, then $T\left[x_{1}\right]$ again, then $T\left[x_{2}\right]$, then back to $T\left[x_{0}\right]$, and so on, just as in the construction for trees of height 4. By the assumption of Case 1 (which began on page 47 ), $\lim _{s} v_{i, s}$ converges for infinitely many $i$, so $w_{0, s}$ must eventually converge to some $w_{0}$. At the same time, we do the same for the witness node $w_{1, s}$ for $\mathcal{R}_{1}$, looking only at witness nodes in successor trees $T\left[x_{j}\right]$ in which $w_{0, s}$ has never yet been located, and so on by a standard finite-injury process. The following sublemma is now clear:

Sublemma 3.31 For every $e, w_{e}=\lim _{s} w_{e, s}$ exists and $T\left[w_{e}\right] \hookrightarrow I_{1}$ and $T\left[w_{e}\right] \neq I_{1}$.

We build $T^{\prime}$ by copying $T$ at each stage, with the following provision. Find each $e \leq s$ such that $w_{e, s}$ is defined and $\varphi_{e, s}\left(w_{e, s}\right) \downarrow\left(\right.$ say $\left.y=\varphi_{e, s}\left(w_{e, s}\right)\right)$ and $f_{s}^{-1}(y)$ lies at level 2 in $T_{s}$. If there is no such $e$, simply extend $f_{s}$ to $f_{s+1}$ by adding new elements to $T_{s+1}^{\prime}$. If there is such an $e$, then for each such $e$, we check whether there exists a basic embedding of $T_{s}\left[f_{s}^{-1}(y)\right]$ into $I_{1}$ (recall that this is a computable condition using our nice copy of $I_{1}$ ). If no such embedding exists, then we are assured that $T\left[f_{s}^{-1}(y)\right] \nrightarrow I_{1}$, so if $w_{e, s}=w_{e}$, then $\mathcal{R}_{e}$ will be satisfied. If such an embedding does exist, then we add elements to $T_{s+1}^{\prime}[y]$ to make it a copy of $I_{1}$, and add more new elements to $T_{s+1}^{\prime}$ to be the new image of $T_{s}\left[f_{s}^{-1}(y)\right]$ under $f_{s+1}$. Hence $T^{\prime}\left[\varphi_{e}\left(w_{e, s}\right)\right] \cong I_{1}$, so if $w_{e, s}=w_{e}$, we have satisfied $\mathcal{R}_{e}$. Notice that $f_{s+1}$ is no longer onto 
$T_{s+1}^{\prime}$ since we have added a copy of $I_{1}$. However, we know that $I_{1}$ occurs infinitely often as a successor tree above $x_{i}$ in $T$, so $T$ and $T^{\prime}$ are still isomorphic. $\mathcal{R}_{i}$ is injured if $w_{e, s+1} \neq w_{e, s}$ for some $e<i$.

As with the case for trees of height 4 , there are two minor modifications necessary for this strategy. First, we give $2 e+1$ many witnesses to each $\mathcal{R}_{e}$ strategy and force this requirement to respect $\mathcal{N}_{u}$ and $\mathcal{M}_{u}$ for $u<e$. That is, $\mathcal{R}_{e}$ is forbidden to use $w_{e, s}$ if $u \prec f_{s}^{-1}\left(\varphi_{e, s}\left(w_{e, s}\right)\right)$ for some $u<e$ or if $\varphi_{e, s}\left(w_{e, s}\right)<e$. The reason for explicitly adding the $\mathcal{M}_{u}$ requirements will become clear below when we discuss the general case where our set of nodes $X$ from the statement of Lemma 3.19 is $\Delta_{2}^{0}$ rather than computable. Second, it is possible that $\varphi_{e}\left(w_{e, s}\right)=y$ lies in a copy of $I_{1}$ in $T_{1}$. In this case, if $w_{e}=w_{e, s}$, then $\mathcal{R}_{e}$ is satisfied without any further action.

We end this case with some comments on how to combine the $\Delta_{2}^{0}$ approximation for the $x_{i}$ elements with the strategy just described. Suppose we have an element $x$ which we think is an $x_{i}$ element and we diagonalize at stage $s$ using a successor of $x$. This means that we want to create a copy of $I_{1}$ as a successor tree of $T^{\prime}\left[f_{s}(x)\right]$. The worry is that if we put $I_{1}$ down all at once, it may turn out that $x$ is not one of the $x_{i}$ elements, and even worse, that $x$ has no successor tree of type $I_{1}$. Such an outcome could destroy our isomorphism. Therefore, we fix an approximation $I_{1, s}$ to $I_{1}$ by finite subtrees. Instead of putting down all of $I_{1}$ at once as a successor tree of $T^{\prime}\left[f_{s}(x)\right]$, we build up $I_{1}$ by putting down $I_{1, t}$ at stage $t \geq s$. Furthermore, before putting down $I_{1, t}$, we check for either

1. evidence that $x$ is not an $x_{i}$ element; or

2. a successor $y$ of $x$ such that $y$ is bigger than any number seen so far in the construction and $I_{1, t}$ embeds into $T[y]$.

This search procedure must terminate since if $x$ is an $x_{i}$ element, then $x$ has infinitely many successor trees of type $I_{1}$. The reason for including clause (2) in the search is that if we find evidence that $x$ is not an $x_{i}$ element at stage $t+1$, then we can use the successor tree $T[y]$ found at stage $t$ to map to the copy of $I_{1, t}$ currently sitting as a successor tree of $T_{t}^{\prime}\left[f_{t}(x)\right]$. Since we can correct any mistakes caused by the $\Delta_{2}^{0}$ approximation, it is straightforward to add it in as a formal part of the above construction. Finally, notice that when we take into account this $\Delta_{2}^{0}$ approximation procedure, we can have elements $y \in T^{\prime}$ which leave the range of $f$ and later return to the range of $f$. This is the reason why we need to explicitly add the $\mathcal{M}_{u}$ requirements into this construction.

Case 2. If Case 1 (which began on page 47) does not apply, then there are only finitely many $T\left[x_{i}\right]$ in which some finite-appearing successor tree embeds into any of $I_{1}, \ldots, I_{p}$. We assume finitely much information, namely the roots of those finitely many successor trees, and ignore them in our construction, defining the elements $x_{i}$ to be those nodes at level 1 in $T$ which are not roots of these finitely many successor trees.

Consider any embedding $T\left[x_{i}\right] \hookrightarrow T\left[x_{j}\right]$ among these nodes at level 1 in $T$. Since no successor tree which occurs finitely often in $T\left[x_{i}\right]$ can embed into any of $I_{1}, \ldots, I_{p}$, we know that they must embed into the successor trees which occur finitely often in $T\left[x_{j}\right]$. Of course, 
the same relation holds for embeddings $T\left[x_{j}\right] \hookrightarrow T\left[x_{i}\right]$. Therefore, if we let $T_{i}$ (respectively $T_{j}$ ) be the tree formed by taking a root and adjoining all of the finitely occurring successor trees in $T\left[x_{i}\right]$ (in $T\left[x_{j}\right]$ respectively), then we have $T_{i} \equiv T_{j}$.

Sublemma 3.32 Fix $f: T_{i} \hookrightarrow T_{j}$ and $g: T_{j} \hookrightarrow T_{i}$. Then for any $u \in T_{i}$ at level 1 , there $i s$ a $v \in T_{j}$ at level 1 such that $T_{i}[u] \equiv T_{j}[v]$, and vice versa.

Proof. Fix any $u_{1} \in T_{i}$ at level 1 and let $v_{1} \in T_{j}$ be such that $v_{1}$ is at level 1 and $f$ maps $T_{i}\left[u_{1}\right] \hookrightarrow T_{j}\left[v_{1}\right]$. If $g$ maps $T_{j}\left[v_{1}\right] \hookrightarrow T_{i}\left[u_{1}\right]$, then we are done, so assume this does not happen. Let $u_{2} \neq u_{1}$ be at level 1 in $T_{i}$ such that $g$ maps $T_{j}\left[v_{1}\right] \hookrightarrow T_{i}\left[u_{2}\right]$.

Fix $v_{2} \in T_{j}$ at level 1 such that $f$ maps $T_{i}\left[u_{2}\right] \hookrightarrow T_{j}\left[v_{2}\right]$. We claim that $v_{2} \neq v_{1}$. For a contradiction, suppose that $v_{2}=v_{1}$. Then $f$ maps $T_{i}\left[u_{2}\right] \hookrightarrow T_{j}\left[v_{2}\right]=T_{j}\left[v_{1}\right]$ and $g$ maps $T_{j}\left[v_{1}\right] \hookrightarrow T_{i}\left[u_{2}\right]$. Therefore, $T_{i}\left[u_{2}\right] \equiv T_{j}\left[v_{1}\right]$ which means $f\left(u_{2}\right)=v_{2}=v_{1}$. But, $f$ also maps $T_{i}\left[u_{1}\right] \hookrightarrow T_{j}\left[v_{1}\right]$, so $v_{1} \prec f\left(u_{1}\right)$. Together, these statements imply that $f\left(u_{2}\right) \prec f\left(u_{1}\right)$, which contradicts the fact that $u_{1}$ and $u_{2}$ are incomparable nodes at level 1 in $T_{i}$.

We now check whether $g$ maps $T_{j}\left[v_{2}\right] \hookrightarrow T_{i}\left[u_{1}\right]$. If so, then we have

$$
T_{i}\left[u_{1}\right] \hookrightarrow T_{j}\left[v_{1}\right] \hookrightarrow T_{i}\left[u_{2}\right] \hookrightarrow T_{j}\left[v_{2}\right] \hookrightarrow T_{i}\left[u_{1}\right]
$$

In this case, $T_{i}\left[u_{1}\right] \equiv T_{i}\left[u_{2}\right] \equiv T_{j}\left[v_{1}\right] \equiv T_{j}\left[v_{2}\right]$ with $f\left(u_{1}\right)=v_{1}, f\left(u_{2}\right)=v_{2}, g\left(v_{1}\right)=u_{2}$ and $g\left(v_{2}\right)=u_{1}$, which means we are done. Otherwise, fix $u_{3} \in T_{i}$ at level 1 such that $u_{3} \neq u_{1}$ and $g$ maps $T_{j}\left[v_{2}\right] \hookrightarrow T_{i}\left[u_{3}\right]$.

We claim that $u_{3} \neq u_{2}$. For a contradiction, suppose that $u_{3}=u_{2}$. Then $g$ maps $T_{j}\left[v_{2}\right] \hookrightarrow$ $T_{i}\left[u_{3}\right]=T_{i}\left[u_{2}\right]$ and $f$ maps $T_{i}\left[u_{2}\right] \hookrightarrow T_{j}\left[v_{2}\right]$. Therefore, $T_{j}\left[v_{2}\right] \equiv T_{i}\left[u_{2}\right]$ and so $g\left(v_{2}\right)=u_{2}$. But, $g$ also maps $T_{j}\left[v_{1}\right] \hookrightarrow T_{i}\left[u_{2}\right]$ which means $u_{2} \prec g\left(v_{1}\right)$. Therefore, $g\left(v_{2}\right) \prec g\left(v_{1}\right)$. This contradicts the fact that $v_{1}$ and $v_{2}$ are incomparable nodes at level 1 in $T_{j}$.

We next let $v_{3}$ be a node at level 1 in $T_{j}$ such that $f$ maps $T_{i}\left[u_{3}\right] \hookrightarrow T_{j}\left[v_{3}\right]$. We claim that $v_{3}$ is not equal to either $v_{1}$ or $v_{2}$. For a contradiction, suppose $v_{3}=v_{1}$. Then $f$ maps $T_{i}\left[u_{3}\right] \hookrightarrow T_{j}\left[v_{3}\right]=T_{j}\left[v_{1}\right]$ and the composition $g f g$ maps $T_{j}\left[v_{1}\right] \hookrightarrow T_{i}\left[u_{3}\right]$. Therefore, $T_{i}\left[u_{3}\right] \equiv T_{j}\left[v_{1}\right]$ and $f\left(u_{3}\right)=v_{3}=v_{1}$. But, we also know that $f$ maps $T_{i}\left[u_{1}\right] \hookrightarrow T_{j}\left[v_{1}\right]$ which implies $v_{1} \prec f\left(u_{1}\right)$. Together, these statements say that $f\left(u_{3}\right) \prec f\left(u_{1}\right)$ which contradicts the fact that $u_{1}$ and $u_{3}$ are incomparable nodes at level 1 in $T_{i}$. The argument that $v_{3} \neq v_{2}$ is similar.

We continue by induction. Suppose $u_{1}, \ldots, u_{n}$ are pairwise distinct nodes at level 1 in $T_{i}$ and $v_{1}, \ldots, v_{n}$ are pairwise distinct nodes at level 1 in $T_{j}$ such that $f$ maps $T_{i}\left[u_{k}\right] \hookrightarrow T_{j}\left[v_{k}\right]$ (for $k \leq n$ ) and $g$ maps $T_{j}\left[v_{k}\right] \hookrightarrow T_{i}\left[u_{k+1}\right]$ (for $k<n$ ). We check if $g$ maps $T_{j}\left[v_{n}\right] \hookrightarrow T_{i}\left[u_{1}\right]$. If so, then

$$
T_{i}\left[u_{1}\right] \equiv \cdots \equiv T_{i}\left[u_{n}\right] \equiv T_{j}\left[v_{1}\right] \equiv \cdots \equiv T_{j}\left[v_{n}\right]
$$

and $f\left(u_{k}\right)=v_{k}$ (for $\left.k \leq n\right), g\left(v_{k}\right)=u_{k+1}($ for $k<n)$ and $g\left(v_{n}\right)=u_{1}$. In this case, we are done.

Otherwise, we fix $u_{n+1} \neq u_{1}$ at level 1 in $T_{i}$ such that $g$ maps $T_{j}\left[v_{n}\right] \hookrightarrow T_{i}\left[u_{n+1}\right]$. We argue as above that $u_{n+1} \neq u_{k}$ for all $k \leq n$. We let $v_{n+1}$ be a node at level 1 in $T_{j}$ such that $f$ 
maps $T_{i}\left[u_{n+1}\right] \hookrightarrow T_{j}\left[v_{n+1}\right]$. We argue as above that $v_{n+1} \neq v_{k}$ for all $k \leq n$. We are now in position to continue the induction.

Since there are only finitely many nodes at level 1 in $T_{i}$ and $T_{j}$, this process must come to an end. Therefore, we get our result in the end. Notice that this proof shows that the number of nodes at level 1 in $T_{i}$ is less that or equal to the number of nodes at level 1 in $T_{j}$. If we switch the roles of $T_{i}$ and $T_{j}$, we get that for any $u \in T_{j}$ at level 1 , there is a $v \in T_{i}$ at level 1 such that $T_{j}[u] \equiv T_{i}[v]$. Therefore $T_{i}$ and $T_{j}$ have the same number of nodes at level 1.

This sublemma tells us that each $T\left[x_{i}\right]$ has the same number of finitely occurring successor trees. Let $y_{1}, \ldots, y_{q}$ be the roots of the finitely occurring successor trees in $T\left[x_{0}\right]$. We define an equivalence relation by $y_{i} \sim y_{j}$ if $T\left[y_{i}\right] \equiv T\left[y_{j}\right]$. The embedding relation $\hookrightarrow$ between these classes is well defined. Furthermore, we know by Sublemma 3.32 that the classes defined in a similar way for the finitely occurring successor trees of any other $T\left[x_{i}\right]$ are exactly the same and each equivalence class has exactly the same size.

For each $y_{n}$, fix a finite tree $S_{n} \subset T\left[y_{n}\right]$ such that $S_{n}$ does not embed into any of the infinite types $I_{1}, \ldots, I_{p}$. Furthermore, for each $m \leq q$ such that $T\left[y_{n}\right] \hookrightarrow T\left[y_{m}\right]$, we extend $S_{n}$ to a finite tree such that $S_{n} \nrightarrow T\left[y_{m}\right]$. Of course, these finite trees $S_{n}$ have the same properties relative to the finitely occurring successor trees above any other $T\left[x_{i}\right]$. Therefore, we can use these trees to identify the finitely many finitely occurring successor trees above each node $x_{i}$. That is, for each $x_{i}$ we look for the appropriate number of successors $y$ such that $T[y]$ contains one of these finite trees. Furthermore, given a $\Delta_{2}^{0}$ procedure to identify the nodes $x_{i}$, there is a $\Delta_{2}^{0}$ procedure to identify the successors $y$ of $x_{i}$ for which $T[y]$ is a finitely occurring successor tree and to determine which equivalence class $T[y]$ belongs to.

There are infinitely many different isomorphism types among the trees $\left\{T\left[x_{i}\right]: i \in \omega\right\}$. Since they all have the same infinite-occurring isomorphism types, we may fix an equivalence class $\mathcal{E}$ such that $\{T[y]: y \in T \wedge y \in \mathcal{E}\}$ contains infinitely many different isomorphism types. (Here we are interpreting $\mathcal{E}$ as a class including successor nodes from each of the trees $T\left[x_{i}\right]$. By the comments above, we have a $\Delta_{2}^{0}$ procedure to identify $y \in \mathcal{E}$.) Moreover, every $T[y]$ with $y \in \mathcal{E}$ is of height at most $n-2$, since $\operatorname{level}_{T}(y)=2$. Our construction of $T^{\prime}$ therefore uses induction on height, for which we regard the height 4 case given above as the base case. We identify elements of $\mathcal{E}$ above the various $x_{i}$ and do the same construction on them that we did for a tree $T$ with $h t(T) \leq n-1$.

To be more specific, suppose that $\mathcal{E}=\left\{z_{i} \mid i \in \omega\right\}$. We begin the proof of Lemma 3.19 again, using the $z_{i}$ elements in place of the $x_{i}$ elements. The only change in the hypothesis of the lemma is that each $z_{i}$ is at level 2 rather than at level 1 . However, this change does not affect the argument at all. That is, the fact that $T\left[z_{i}\right] \equiv T\left[z_{j}\right]$ implies that each $T\left[z_{i}\right]$ has the same infinitely occurring successor trees. We denote the isomorphism types of these trees by $J_{1}, \ldots, J_{b}$. If there are infinitely many $i$ for which $T\left[z_{i}\right]$ has a finitely occurring successor tree which embeds in one of $J_{1}, \ldots, J_{b}$, then we may assume without loss of generality that there are infinitely many $i$ which work with $J_{1}$. We run exactly the same argument as in Case 1 , looking for appropriate successors of the $z_{i}$ with which to diagonalize. Otherwise, if there are 
not infinitely many such $i$, we are back in Case 2 and we repeat this process over again with nodes at level 3. Since $T$ has finite height, this process must stop. This completes the case of a height $n$ tree, and also completes the proof of Lemma 3.19.

Proof of Proposition 3.1. Let $T$ be as in the statement of Proposition 3.1. Let $r$ be the root of $T$, and let $x_{0}, x_{1}, \ldots$ be the immediate successors of $r$ in $T$. Then every node above $r$ in $T$ is of finite type. Since $r$ is not of finite type, there must be infinitely many of these successor trees. We consider the three ways in which $r$ could fail to be of finite type as in Definition 1.7 .

First, suppose there is an isomorphism type $I$ which occurs infinitely often as a successor tree of $r$ and which does not have strongly finite type. We split into two cases. If there are only finitely many isomorphism types of successor trees of $r$ which embed into $I$, then Lemma 3.10 shows that $T$ is not computably categorical. If there are infinitely many isomorphism types of successor trees of $r$ which embed into $I$, then Lemma 3.16 implies that $T$ is not computably categorical. Therefore, we can assume that any isomorphism type which occurs infinitely as a successor tree of $r$ has strongly finite type.

Second, suppose there exist distinct isomorphism types $I_{0}$ and $I_{1}$ such that each occurs infinitely often as a successor tree to $r$ and $I_{0} \hookrightarrow I_{1}$. Since we can assume $I_{0}$ and $I_{1}$ have strongly finite type and they are not isomorphic, we must have $I_{1} \nrightarrow I_{0}$ by Lemma 2.10. We can now apply Lemma 3.15. Let the indices $i$ be those for which $T\left[x_{i}\right] \cong I_{0}$ and let the indices $j$ be those for which $T\left[x_{j}\right] \cong I_{1}$. There are infinitely many such indices $i$ and $j, T\left[x_{i}\right] \hookrightarrow I_{0}$, $I_{0} \hookrightarrow T\left[x_{j}\right]$ and $T\left[x_{j}\right] \nrightarrow I_{0}$. Therefore, Lemma 3.15 proves that $T$ is not computably categorical. We can now assume that there is no embedding between isomorphism types which occur infinitely often as successor trees of $r$. By Lemma 3.5, we know that there can only be finitely many isomorphism types which occur infinitely often as successor trees of $r$.

Finally, let $\mathcal{T}$ be the set of isomorphism types which occur among the successor trees of $r$. It could be that $\mathcal{T}$ is infinite. We split into two cases. If there is an infinitely occurring isomorphism type $I$ for which $I^{\prime} \hookrightarrow I$ for infinitely many $I^{\prime} \in \mathcal{T}$, then we can apply Lemma 3.16. Otherwise, the finitely many isomorphism types which occur infinitely often each have only finitely many isomorphism types from $\mathcal{T}$ which embed into them. This situation is exactly the hypothesis for Lemma 3.18. Thus we have proved Proposition 3.1.

In the cases above in which we constructed a $\Delta_{2}^{0}$ isomorphism $f$ between $T$ and $T^{\prime}$, the computable dimension of $T$ must be $\omega$ by Goncharov [10]. However, we can see this more directly (and prove it in the remaining cases) simply be rewriting the positive requirements:

$$
\mathcal{R}_{\langle e, i\rangle}: \quad \varphi_{e} \text { one-one and total } \Longrightarrow\left[\left(\exists w_{e} \in T_{i}\right) T_{i}\left[w_{e}\right] \not T^{\prime}\left[\varphi_{e}\left(w_{e}\right)\right]\right] \text {. }
$$

Here $\left\{T_{i}\right\}$ is assumed to be a finite sequence of computable trees isomorphic to $T$, and the $T^{\prime}$ which we construct to satisfy these requirements will be of a different computable isomorphism class from each of them. In the original construction, $T$ actually served a dual purpose, as both the template for $T^{\prime}$ and the computable isomorphism type to be avoided. Here we always use $T_{0}$ as the template, but diagonalize simultaneously against all the $T_{i}$. 


\section{Induction}

In this section, we prove the second half of Theorem 1.8, that trees which are not of finite type cannot be computably categorical, and indeed must have computable dimension $\omega$. Section 2 established the converse of this statement, and Section 3 enables us to use induction to prove the following proposition.

Proposition 4.1 Let $T$ be a tree of finite height but not of finite type. Then $T$ is not computably categorical.

Proof. The proof uses induction on the height $n$ of $T$. The base case $n=2$ is trivial, since every tree of height $\leq 2$ is of finite type. Let $r$ be the root of $T$, with immediate successors $x_{0}, x_{1}, \ldots$ If every node $x_{i}$ is of finite type, then Proposition 3.1 shows that $T$ has infinite computable dimension. So we may suppose that some isomorphism type $I_{0}$ appearing above $r$ is not of finite type. (Without loss of generality we assume that $T\left[x_{0}\right] \cong I_{0}$.) By the inductive hypothesis, $I_{0}$ must not be computably categorical, so there is a computable tree $U$ which is isomorphic to $T\left[x_{0}\right]$ but not computably isomorphic to it, and we may take the domain of $U$ to be the computable set $T\left[x_{0}\right]$. Let $V$ be identical to $T$, only with $U$ in place of $T\left[x_{0}\right]$. Then $V$ is computable and isomorphic to $T$.

Now we assume for a contradiction that $T$ is computably categorical. Then there must exist a computable isomorphism $\varphi$ from $V$ to $T$, which must map $U$ to some other successor tree $T\left[x_{j}\right]$ computably isomorphic to $U$. (Hence $j \neq 0$.) Moreover, $\varphi$ would then have to map $T\left[x_{j}\right]$ (which is also a successor tree in $V$ ) to yet another successor tree $T\left[x_{k}\right]$ computably isomorphic to $U$, and so on. Therefore, the isomorphism type $I_{0}$ must appear infinitely often above $r$ in $T$.

Moreover, since $I_{0}$ appears infinitely often above $r$, we can build another computable tree isomorphic to $T$, simply by adding any computable copy of $I_{0}$ as a new successor tree above $r$. Since this copy can be of any computable isomorphism type for $I_{0}$, the same argument as above shows that every computable isomorphism type of $I_{0}$ must appear infinitely often as a successor tree above $r$. Indeed, under the assumption that $T$ is computably categorical, we see that for each such computable isomorphism type this process would yield an infinite c.e. set of roots of successor trees of that computable isomorphism type.

Since $I_{0}$ is not computably categorical, we have at least two of these c.e. sets, say $C_{1}$ and $C_{2}$. The idea is to use elements of $C_{1}=\left\{w_{0}, w_{1}, \ldots\right\}$ as witness elements when we build $T^{\prime}$. We wait until $\varphi_{e, s}\left(w_{e}\right)$ converges, and then redefine the $\Delta_{2}^{0}$-isomorphism $f: T \rightarrow T^{\prime}$ so that from stage $s$ on, $T^{\prime}\left[\varphi_{e}\left(w_{e}\right)\right]$ is built computably isomorphic to $T\left[y_{e}\right]$, where $C_{2}=\left\{y_{0}, y_{1} \ldots\right\}$. (Namely, define $f_{s+1}\left(f_{s}^{-1}\left(\varphi_{e}\left(w_{e}\right)\right)\right)=y_{e}$.) The difficulty is that at the stage $s$ at which $\varphi_{e}\left(w_{e}\right)$ converges, we do not know if $T_{s}^{\prime}\left[\varphi_{e}\left(w_{e}\right)\right]$ embeds into $I_{0}$ or not, since $f_{s}^{-1}\left(\varphi_{e}\left(w_{e}\right)\right)$ may or may not lie in a successor tree in $T$ isomorphic to $I_{0}$. To handle this difficulty, we appeal to a corollary of Kruskal's Theorem.

Corollary 4.2 Let $\left\{S_{i}: i \in \omega\right\}$ be an infinite set of finite trees. Then there exists $m \in \omega$ such that for every $j$ there is an $i \leq m$ such that $S_{i} \hookrightarrow S_{j}$. 
Proof. If the set $\left\{S_{j}:(\forall i<j)\left[S_{i} \hookrightarrow S_{j}\right]\right\}$ were infinite, it would contradict Kruskal's Theorem. Hence we may take $m$ to be the greatest index in this set. The corollary follows by an easy induction on the indices $>m$.

Let $J$ be the set of all finite trees $S$ which do not embed into $I_{0}$. Then Corollary 4.2 yields a finite subset $\mathcal{S} \subseteq J$ such that for every $S \in J$ there is some $S^{\prime} \in \mathcal{S}$ such that $S^{\prime} \hookrightarrow S$. Moreover, no $S^{\prime} \in \mathcal{S}$ embeds into $I_{0}$.

The witness elements for our construction will be the nodes $w_{e}$ described above. Since the set $C_{1}$ is infinite and computably enumerable, we need not use $\Delta_{2}^{0}$ guessing, either for them or for the corresponding nodes $y_{e} \in C_{2}$. (Technically, we will use $\Delta_{2}^{0}$-guessing, but with a simple method of renaming the elements of $C_{1}$ and $C_{2}$.)

A requirement $\mathcal{R}_{e}$ requires attention at stage $s$ if $s$ is the least stage such that $\varphi_{e, s}\left(w_{e}\right)$ converges to some $w_{e}^{\prime} \in T_{s}^{\prime}$. At each stage $s$, we simply extend $f_{s}$ to $f_{s+1}$ mapping $T_{s+1}$ to $T_{s+1}^{\prime}$ by adding fresh elements to $T_{s+1}^{\prime}$ as needed, except on those successor trees $T_{s}\left[w_{e}\right]$ such that $\mathcal{R}_{e}$ requires attention. For those $e$, we search for the least $t \geq s$ such that one of the following holds:

1. $\operatorname{level}_{T_{t}}\left(f_{s}^{-1}\left(w_{e}^{\prime}\right)\right) \neq 1$; or

2. some $S^{\prime} \in \mathcal{S}$ embeds into $T_{t}\left[f_{s}^{-1}\left(w_{e}^{\prime}\right)\right]$; or

3. $T_{s}^{\prime}\left[w_{e}^{\prime}\right]$ embeds into $T_{t}\left[y_{e}\right]$.

If either (1) or $(2)$ holds, then again we simply extend $f_{s}$ to $f_{s+1}$ on $T_{s+1}\left[w_{e}\right]$ by adding fresh elements to $T_{s+1}^{\prime}$, without redefining $f_{s+1}$ on any nodes. However, if (3) holds, then we may need to redefine $f$.

The idea of the redefinition of $f$ is as follows. Let $a=f_{s}^{-1}\left(w_{e}^{\prime}\right)$. Currently, using $f_{s}, T^{\prime}\left[w_{e}^{\prime}\right]$ is being built by copying $T[a]$ and $T^{\prime}\left[f_{s}\left(y_{e}\right)\right]$ is being built by copying $T\left[y_{e}\right]$. We use the embedding in (3) to define $f_{s+1}$ so that $T^{\prime}\left[w_{e}^{\prime}\right]$ begins copying $T\left[y_{e}\right]$ and $T^{\prime}\left[f_{s}\left(y_{e}\right)\right]$ begins copying $T[a]$. This successfully diagonalizes because $T\left[w_{e}\right]$ and $T\left[y_{e}\right]$ are not computably isomorphic. Before performing this switch, we need to check that no higher priority requirements will be injured. We ask first whether $w_{e}^{\prime}$ lies in the set

$$
P_{e, s}=\left\{f_{s}\left(y_{0}\right), \ldots f_{s}\left(y_{e-1}\right)\right\} \cup\left\{w_{i}^{\prime}: i<e \& \varphi_{i, s}\left(w_{i}\right) \downarrow=w_{i}^{\prime}\right\} .
$$

If it does, then we cannot redefine $f$ without possibly injuring some $\mathcal{R}_{i}$ of higher priority, so instead we eliminate $w_{e}$ from our enumeration of $C_{1}$ and pick $w_{s+1}$ to be the witness node for $\mathcal{R}_{e}$. (At future stages, we will refer to this node as $w_{e}$.) However, if this elimination has already happened $2 e$ times for different values of $w_{e}$ at previous stages, and the elements of $P_{e, s}$ have not changed since those stages, then we need not perform the elimination again (nor redefine $f$ at all), since in this case $\varphi_{e}$ must map all $2 e+1$ of those different values of $w_{e}$ into $P_{e, s}$ and hence cannot be one-to-one.

If $w_{e}^{\prime} \notin P_{e, s}$, then we may proceed without injuring any higher-priority requirement. (If $w_{e}^{\prime}=f_{u}\left(y_{j}\right)$ for some $j>e$ at some later stage $u$, we will simply ignore that $y_{j}$ and renumber 
$C_{2}$ with the element $y_{j+1}$ as $y_{j}$ instead, thereby possibly injuring a lower-priority requirement once.) Let $g$ be the embedding of $T_{s}^{\prime}\left[w_{e}^{\prime}\right]$ into $T_{t}\left[y_{e}\right]$. (We may assume $g\left(w_{e}^{\prime}\right)=y_{e}$.) Define $f_{s+1}(x)=g^{-1}(x)$ for all $x$ in the image of $g$, and add fresh elements to $T_{s+1}^{\prime}$ to be the range of all of $T\left[y_{e}\right]$ under $f_{s+1}$. For all sufficiently large elements $x \in T\left[y_{e}\right]$, we may take $f_{s+1}(x)=x$. Thus $f_{s+1}$ now maps $T\left[y_{e}\right]$ to $T^{\prime}\left[w_{e}^{\prime}\right]$.

We also must redefine $f_{s+1}$ on the set $A=f_{s}^{-1}\left(T_{s}^{\prime}\left[w_{e}^{\prime}\right]\right)$. Now since $f_{s}$ is an isomorphism from $T_{s}$ to $T_{s}^{\prime}, A \cong T_{s}^{\prime}\left[w_{e}^{\prime}\right]$ must embed into $I_{0}$ via a lifting of the same $g$, so we may find an embedding $h$ of $A$ into $T\left[y_{e}\right]$. We add enough elements of $T\left[y_{e}\right]$ to $T_{s+1}$ so that $A \hookrightarrow T_{s+1}\left[y_{e}\right]$, then combine this embedding with $f_{s}: T_{s}\left[y_{e}\right] \hookrightarrow T_{s}^{\prime}\left[f_{s}\left(y_{e}\right)\right]$, adding fresh elements to $T_{s+1}$ as needed, to define $f_{s+1}$ on $A$. Thus $f_{s+1}$ maps $T\left[f_{s}^{-1}\left(w_{e}^{\prime}\right)\right]$ to $T^{\prime}\left[f_{s}\left(y_{e}\right)\right]$. This completes the construction.

With the redefinition, we see that now $T\left[y_{e}\right]$ is not only isomorphic to $T^{\prime}\left[w_{e}^{\prime}\right]$, but actually computably isomorphic to it via $f$, since $f$ is the identity map on cofinitely much of $T\left[y_{e}\right]$. If $\varphi_{e}$ were an isomorphism from $T$ to $T^{\prime}$, then $f^{-1} \circ \varphi_{e}$ would be a computable isomorphism from $T\left[w_{e}\right]$ onto $T\left[y_{e}\right]$, which is impossible, by our choice of $w_{e}$ and $y_{e}$. Hence $\mathcal{R}_{e}$ is satisfied.

Moreover, if either (1) or (2) holds for $w_{e}^{\prime}$, then $\mathcal{R}_{e}$ must again be satisfied. This is clear for (1), since level ${ }_{T}\left(w_{e}\right)=1$. If (2) holds, then some $S \in \mathcal{S}$ embeds into $T^{\prime}\left[w_{e}^{\prime}\right]$, but not into $T\left[w_{e}\right]$ (by our choice of $\mathcal{S}$ ). Hence clearly $\mathcal{R}_{e}$ is satisfied.

We must show that when we search for a stage $t$ in the construction, we do eventually find one. Suppose level $T_{T^{\prime}}\left(w_{e}^{\prime}\right)=1$, and suppose that no $S \in \mathcal{S}$ embeds into $T\left[f_{s}^{-1}\left(w_{e}^{\prime}\right)\right]$. By our choice of $\mathcal{S}$, this guarantees that every finite subtree of $T\left[f_{s}^{-1}\left(w_{e}^{\prime}\right)\right]$ embeds into $I_{0}$. But $T_{s}^{\prime}\left[w_{e}^{\prime}\right]$ is isomorphic to $T_{s}\left[f_{s}^{-1}\left(w_{e}^{\prime}\right)\right]$, hence must embed into $I_{0}$. Since $T\left[y_{e}\right] \cong I_{0}$, we will eventually find a stage $t$ and an embedding satisfying (3).

The redefinition process does no injury to any other $\mathcal{R}_{e}$. The only possibility for injury among the requirements occurs when elements of $C_{1}$ or $C_{2}$ must be ignored or renamed, as described above, and when this happens each requirement respects the higher-priority requirements, so ultimately each $\mathcal{R}_{e}$ is satisfied.

Moreover, redefinition of $f$ can only occur finitely often on any $T[x]$, and redefinition of $f^{-1}$ can only occur finitely often on any $T^{\prime}\left[x^{\prime}\right]$, since each requirement is injured only finitely often. (Our care in making $f_{s}\left(w_{e}\right) \notin P_{e, s}$ ensured this for $f^{-1}$.) Hence $f$ is a bijection between $T$ and $T^{\prime}$. Since our redefinitions always respected the partial order we were building on $T^{\prime}$, $T^{\prime}$ is computable and $f$ is an isomorphism. But since each $\mathcal{R}_{e}$ holds, there is no computable isomorphism from $T$ to $T^{\prime}$, contradicting our assumption that $T$ was computably categorical.

Corollary 4.3 Every computable tree $T$ of finite height but not of finite type must have infinite computable dimension.

Proof. Because we proved Proposition 4.1 by contradiction, we do not know if there are two computable copies of $T$ which are $\Delta_{2}^{0}$-isomorphic but not computably isomorphic. Therefore, we cannot apply Goncharov's result that a pair of computable structures which are $\Delta_{2}^{0}$ isomorphic but not computably isomorphic must have computable dimension $\omega$. Instead, the 
proof proceeds by induction on the height of $T$. Assume $T$ is a tree of finite height which does not have finite type. If every successor tree in $T$ has finite type, then we are done by the results in Section 3. Otherwise, we fix $y \in T$ at level 1 such that $T[y]$ does not have finite type. By the induction hypothesis, $T[y]$ must have infinite computable dimension.

For a contradiction, assume that $T$ has finite computable dimension $m$. Fix representatives $T^{0}, \ldots, T^{m-1}$ of the computable isomorphism classes of $T$ and fix nodes $y^{i} \in T^{i}$ at level 1 such that $T[y] \cong T^{i}\left[y^{i}\right]$. To run a diagonalization argument as above, we need to find appropriate c.e. sets $C^{i}$ in $T^{i}$ and $C$ in $T$. We define these sets and specify their exact properties below.

First, we define $C^{i}$ for a fixed $i<m$. Let $x_{e}^{i}$ denote the nodes at level 1 in $T^{i}$ and assume that $y^{i}=x_{0}^{i}$. Let $U_{0}, \ldots, U_{m-1}$ be computable copies of $T^{i}\left[x_{0}^{i}\right]$ defined on the same numbers as $T^{i}\left[x_{0}^{i}\right]$ which are pairwise not computably isomorphic and are not computably isomorphic to $T^{i}\left[x_{0}^{i}\right]$. Let $T_{j}^{i}\left(\right.$ for $j<m$ ) be the computable tree formed by taking $T^{i}$ and replacing $T^{i}\left[x_{0}^{i}\right]$ by $U_{j}$. Since the computable dimension of $T^{i}$ is $m$, one of the $T_{j}^{i}$ trees must be computably isomorphic to $T^{i}$. Without loss of generality, assume it is $T_{0}^{i}$ and fix an isomorphism $f: T_{0}^{i} \rightarrow T^{i}$. $f$ must send $U_{0}$ to some successor tree $T^{i}\left[x_{j_{0}}^{i}\right]$ with $x_{j_{0}}^{i} \neq x_{0}^{i}$. Thus, $T^{i}\left[x_{j_{0}}^{i}\right]$ is a successor tree in $T_{0}^{i}$ and $f$ must send this tree to some $T^{i}\left[x_{j_{1}}^{i}\right]$. Repeating this process, we get a c.e. set of nodes $x_{j_{k}}^{i}$ for successor trees in $T^{i}$ which are computably isomorphic to $U_{0}$. We denote these nodes by $w_{e}^{i}$ and we let $C^{i}$ be the c.e. set of these nodes.

Second, we define $C$. Let $x_{e}$, for $e \in \omega$, denote the nodes at level 1 in $T$ and assume that $y=x_{0}$. Let $V_{0}, \ldots, V_{m-1}$ be computable copies of $T\left[x_{0}\right]$ which are not computably isomorphic to any of the trees $U_{0}, \ldots, U_{m-1}$ used in the definition of $C^{i}$ for any $i$. (It does not matter if we reuse computable isomorphism types when defining $C^{i}$ and $C^{j}$ for $i \neq j$, but we need to have different computable isomorphism types when we define $C$. There are enough computable isomorphism types to accomplish these requirements because $T\left[x_{0}\right]$ has infinite computable dimension.) Let $T_{j}$ (for $j<m$ ) be the computable tree formed by taking $T$ and replacing $T\left[x_{0}\right]$ by $V_{j}$. By the same argument as in the last paragraph, we obtain a c.e. set $C$ of nodes $u_{e}$ at level 1 in $T$ such that $T\left[u_{e}\right]$ is computably isomorphic to (without loss of generality) $V_{0}$. We can sum up the important properties of these c.e. sets by:

- $T^{i}\left[w_{e}^{i}\right] \cong T[y]$ for $e \in \omega$ and $i<m$;

- $T\left[u_{e}\right] \cong T[y]$ for $e \in \omega$;

- $T\left[u_{k}\right] \cong T^{i}\left[w_{e}^{i}\right]$ for $e, k \in \omega$ and $i<m$, but not by a computable isomorphism.

We build $T^{\prime} \cong T$ which is not computably isomorphic to any $T^{i}$ by an argument very similar to the one given above. We build $T^{\prime}$ in stages together with a $\Delta_{2}^{0}$-isomorphism $f$ : $T \rightarrow T^{\prime}$. We index the witnesses in $C$ as $u_{\langle e, i\rangle}$ with $e \in \omega$ and $i<m$ and we use the nodes $w_{e}^{i}$ to diagonalize against $\varphi_{e}$ being an isomorphism from $T^{i}$ to $T^{\prime}$. The strategy to defeat $\varphi_{e}$ and $T^{i}$ is to wait for $\varphi_{e}\left(w_{e}^{i}\right)$ to converge to some $v_{e}^{i} \in T^{\prime}$ at stage $s$. Let $a=f_{s}^{-1}\left(v_{e}^{i}\right)$. We have that $f_{s}$ maps $T_{s}[a]$ to $T_{s}^{\prime}\left[v_{e}^{i}\right]$ and maps $T_{s}\left[u_{\langle e, i\rangle}\right]$ to $T_{s}^{\prime}\left[f_{s}\left(u_{\langle e, i\rangle}\right)\right]$. As above, we either find evidence that we have an easy win or else we find an embedding of $T_{s}^{\prime}\left[v_{e}^{i}\right]$ into $T\left[u_{\langle e, i\rangle}\right]$. In the latter case, we use this embedding to define $f_{s+1}$ so that it swaps the action of $f_{s}$ on the 
successor trees, by making $T^{\prime}\left[v_{e}^{i}\right]$ start to copy $T\left[u_{\langle e, i\rangle}\right]$ and making $T^{\prime}\left[f_{s}\left(u_{\langle e, i\rangle}\right)\right]$ start to copy $T[a]$. As above, this successfully diagonalizes since we know that $T^{i}\left[w_{e}^{i}\right]$ is not computably isomorphic to $T\left[u_{\langle e, i\rangle}\right]$. The formal details of this argument are essentially as above.

We note that for trees $T$ in which nodes at levels $\geq 1$ are not of finite type, these proofs only establish the existence of infinitely many computable isomorphism classes of copies of $T$, without giving us any actual idea how to construct copies in such classes. To construct copies in new classes would require a direct proof in the style of the Lemmas of Section 3, instead of the less-edifying proofs by contradiction in Proposition 4.1 and Corollary 4.3.

\section{$5 \quad \Delta_{n}^{0}$-categoricity}

The goal of this section is to prove the following theorem.

Theorem 5.1 For each $n \geq 1$, there is a computable finite height tree $T$ such that $T$ is $\Delta_{n+1}^{0}$-categorical but not $\Delta_{n}^{0}$-categorical.

We actually prove a slightly stronger statement by considering a more restrictive definition of trees. In this section, we define a tree to be a set $T \subseteq \omega^{<\omega}$ which is closed under initial segments. Such trees are obviously trees in the earlier sense, but they have the additional feature that the successor relation is computable. Therefore, we really establish Theorem 5.1 for computable finite height trees which have a computable successor relation.

Proof. The strategy for this proof is to show by induction on $n \geq 1$ that for any infinite and coinfinite $\Sigma_{n}^{0}$ (if $n$ is odd) or $\Pi_{n}^{0}$ (if $n$ is even) relation $P(x)$, there are computable trees $T_{P}$ and $S_{P}$ such that $T_{P}$ and $S_{P}$ are $\Delta_{n+1}^{0}$-isomorphic but any isomorphism between them computes $P(x)$. For the purposes of presenting a general outline, suppose $n$ is odd, so $T_{P}$ needs to code a $\Sigma_{n}^{0}$ relation $P(x)$.

$T_{P}$ will be $\omega$-branching at the root, with the property that for any node $\tau$ at level $1, T_{P}[\tau]$ has one of two distinct isomorphism types. Trees of one type are called coded $\Sigma_{n}^{0}$ trees and trees of the other type are called uncoded $\Sigma_{n}^{0}$ trees. There will be a computable sequence of nodes $\tau_{x} \in T_{P}$ such that each $\tau_{x}$ is at level 1 and

$$
P(x) \Leftrightarrow T_{P}\left[\tau_{x}\right] \text { is a coded } \Sigma_{n}^{0} \text { tree. }
$$

We will be able to say exactly what the isomorphism type of $T_{P}$ is. It $\omega$-branches at the root and has infinitely many nodes $\tau$ at level 1 for which $T_{P}[\tau]$ is a coded $\Sigma_{n}^{0}$ tree and infinitely many nodes at level 1 for which $T_{P}[\tau]$ is an uncoded $\Sigma_{n}^{0}$ tree. This description of the isomorphism type of $T_{P}$ will allow us to build a computable tree $S_{P}$ which is isomorphic to $T_{P}$, but for which we know exactly which nodes at level 1 correspond to coded $\Sigma_{n}^{0}$ trees and which do not. Therefore, we will be able to compute $P(x)$ from any isomorphism between $S_{P}$ and $T_{P}$. 
To fill in the details of this outline, we first show how to code a $\Sigma_{1}^{0}$ relation. Next, we show how to pass from the coding of a $\Sigma_{1}^{0}$ relation to a coding for a $\Pi_{2}^{0}$ relation, and how to pass from the coding of a $\Pi_{2}^{0}$ relation to the coding of a $\Sigma_{3}^{0}$ relation. Finally, we outline the general procedure for coding a $\Sigma_{n+1}^{0}$ relation from the coding of a $\Pi_{n}^{0}$ relation, and the procedure for coding a $\Pi_{n+1}^{0}$ relation from the coding of a $\Sigma_{n}^{0}$ relation.

First, we show how to code an infinite and coinfinite $\Sigma_{1}^{0}$ relation $P(x)$. Assume that

$$
\forall x(P(x) \Leftrightarrow \exists d R(x, d))
$$

where $R$ is computable. Let $T_{P} \subseteq \omega^{<\omega}$ be the computable set given by the closure of the following conditions under initial segments.

1. For all $n, m,\langle n, m\rangle \in T_{P}$.

2. For all $n, m,\langle n, m, 0\rangle \in T_{P}$ if and only if $m$ is the least number such that $R(n, m)$ holds.

$T_{P}$ has height 4 and is $\omega$-branching at the root. If $P(n)$ holds, then $T_{P}[\langle n\rangle]$ is a tree of height 3 which $\omega$-branches at the root and has a unique node at level 2 (in the restricted tree). We refer to any tree with this isomorphism type as a coded $\Sigma_{1}^{0}$ tree. If $P(n)$ does not hold, then $T_{P}[\langle n\rangle]$ is $\omega$-branching at the root and has no nodes at level 2 . We refer to any tree with this isomorphism type as an uncoded $\Sigma_{1}^{0}$ tree. Notice that the coded and uncoded $\Sigma_{1}^{0}$ trees are not isomorphic.

To give a slightly more general perspective, we need to distinguish coding a $\Sigma_{1}^{0}$ relation and coding a $\Sigma_{1}^{0}$ sentence. To code the $\Sigma_{1}^{0}$ relation $P$, we build a tree $T_{P}$ which is $\omega$-branching and for each $\langle n\rangle \in T_{P}$, we let $T_{P}[\langle n\rangle]$ code the $\Sigma_{1}^{0}$ sentence $P(n)$. That is, we effectively generate a tree $T_{P}[\langle n\rangle]$ which is a coded $\Sigma_{1}^{0}$ tree if the $\Sigma_{1}^{0}$ sentence $P(n)$ is true and is an uncoded $\Sigma_{1}^{0}$ tree if the $\Sigma_{1}^{0}$ sentence $P(n)$ is false.

The isomorphism type of $T_{P}$ is uniquely determined by the following facts: the root of $T_{P}$ is $\omega$-branching, and for every $\tau \in T$ at level $1, T_{P}[\tau]$ is either a coded $\Sigma_{1}^{0}$ tree or an uncoded $\Sigma_{1}^{0}$ tree, with infinitely many of each type. Furthermore, $T_{P}$ has the property that

$$
P(n) \Leftrightarrow T_{P}[\langle n\rangle] \text { is a coded } \Sigma_{1}^{0} \text { tree. }
$$

To see that $T_{P}$ is $\Delta_{2}^{0}$-categorical, suppose that $T$ is computable and isomorphic to $T_{P}$. For any $\tau \in T$ at level $1, T[\tau]$ is a coded $\Sigma_{1}^{0}$ tree if and only if $\exists \sigma_{0}, \sigma_{1}\left(\tau \prec \sigma_{0} \prec \sigma_{1}\right)$. $0^{\prime}$ can determine which nodes $\tau \in T$ are at level 1 and can tell whether $T[\tau]$ is a coded or uncoded $\Sigma_{1}^{0}$ tree. If $T[\tau]$ is a coded $\Sigma_{1}^{0}$ tree, then $0^{\prime}$ can determine the unique node at level 2 in $T[\tau]$. Of course, $0^{\prime}$ can also determine this information in $T_{P}$, so we can easily build the $\Delta_{2}^{0}$ isomorphism.

To see that $T_{P}$ need not be $\Delta_{1}^{0}$-categorical, assume $P(x)$ is noncomputable. Let $S_{P} \subseteq \omega^{<\omega}$ be the closure of the following conditions under initial segments.

1. For all $n, m,\langle n, m\rangle \in S_{P}$.

2. If $n$ is even, then $\langle n, 0,0\rangle \in S_{P}$. 
$S_{P}$ is a computable tree and by the description of the isomorphism type of $T_{P}, S_{P} \cong T_{P}$. In addition,

$$
S_{P}[\langle n\rangle] \text { is a coded } \Sigma_{1}^{0} \text { tree } \Leftrightarrow n \text { is even. }
$$

For any isomorphism $f: T_{P} \rightarrow S_{P}, P(n)$ holds if and only if $f(\langle n\rangle)=\langle m\rangle$ for some even number $m$. Therefore, the fact that $P$ is noncomputable implies that $f$ cannot be $\Delta_{1}^{0}$.

We turn to coding an infinite and coinfinite $\Pi_{2}^{0}$ relation $P(x)$ such that

$$
P(x) \Leftrightarrow \forall c \exists d R(x, c, d),
$$

where $R$ is computable. Let $T_{P} \subseteq \omega^{<\omega}$ be the closure of the following conditions under initial segments. (We give both an informal and a formal description of these conditions. Later, we will trust the reader to fill in the formal descriptions.)

1. $T_{P}$ is $\omega$-branching at the root. Formally, let $\langle 1\rangle \in T_{P}$ and $\left\langle p^{n}\right\rangle \in T_{P}$ for all primes $p$ and all $n \geq 1$. To clarify the coding below, view $\langle 1\rangle$ as $\left\langle 2^{0}\right\rangle$.

2. For all $n$ and $m, T_{P}\left[\left\langle 2^{n}, m\right\rangle\right]$ is the tree defined above for coding the $\Sigma_{1}^{0}$ sentence $\forall c \leq$ $m \exists d R(n, c, d)$. Formally, for all $i,\left\langle 2^{n}, m, i\right\rangle \in T_{P}$ and $\left\langle 2^{n}, m, i, 0\right\rangle \in T_{P}$ if and only if $i$ is the least number such that $\forall c \leq m \exists d \leq i R(n, c, d)$.

3. For all odd primes $p$ and all $n \geq 1, T_{P}\left[\left\langle p^{n}\right\rangle\right]$ consists of $n-1$ copies of the coded $\Sigma_{1}^{0}$ tree and infinitely many copies of the uncoded $\Sigma_{1}^{0}$ tree. Formally, for all $m$ and $i$, $\left\langle p^{n}, m, i\right\rangle \in T_{P}$, and $\left\langle p^{n}, m, i, 0\right\rangle \in T_{P}$ if and only if $i<n-1$.

If $P(n)$ holds, then $T_{P}\left[\left\langle 2^{n}\right\rangle\right]$ is $\omega$-branching at the root, and every node at level 1 (in this restricted tree) is the base of a coded $\Sigma_{1}^{0}$ tree. We call any tree with this isomorphism type a coded $\Pi_{2}^{0}$ tree.

For an odd prime $p$ and $n \geq 1$, the tree $T_{P}\left[\left\langle p^{n}\right\rangle\right]$ is $\omega$-branching at the root and at level 1 has exactly $n-1$ many coded $\Sigma_{1}^{0}$ trees and infinitely many uncoded $\Sigma_{1}^{0}$ trees. We call any tree with this isomorphism type an $(n-1)$-uncoded $\Pi_{2}^{0}$ tree. Notice that if $P(n)$ does not hold, then $T_{P}\left[\left\langle 2^{n}\right\rangle\right]$ is an $m$-uncoded $\Pi_{2}^{0}$ tree for some $m$.

Just as in the $\Sigma_{1}^{0}$ case, we have given a procedure for effectively constructing a tree $T_{P}\left[\left\langle 2^{n}\right\rangle\right]$ from the $\Pi_{2}^{0}$ sentence $P(n)$. This tree is a coded $\Pi_{2}^{0}$ tree if the $\Pi_{2}^{0}$ sentence $P(n)$ holds and it is an $m$-uncoded $\Pi_{2}^{0}$ tree (for some $m$ ) if the $\Pi_{2}^{0}$ sentence $P(n)$ is false. The other trees of the form $T_{P}\left[\left\langle p^{n}\right\rangle\right]$ are added so that the isomorphism type of $T_{P}$ will be independent of the choice of $P$, as long as $P$ is infinite.

We can now describe the isomorphism type of $T_{P}$ precisely. $T_{P}$ is $\omega$-branching at the root and consists of infinitely many coded $\Pi_{2}^{0}$ trees and infinitely many $m$-uncoded $\Pi_{2}^{0}$ trees for each $m$. Furthermore, we have

$$
P(n) \Leftrightarrow T_{P}\left[\left\langle 2^{n}\right\rangle\right] \text { is a coded } \Pi_{2}^{0} \text { tree. }
$$

To see that $T_{P}$ is $\Delta_{3}^{0}$-categorical, fix a computable tree $T$ which is isomorphic to $T_{P}$. For any $\tau \in T$ at level $1, T[\tau]$ is a coded $\Pi_{2}^{0}$ tree if and only if

$$
\forall \sigma\left((\tau \prec \sigma \wedge \neg \exists \delta(\tau \prec \delta \prec \sigma)) \rightarrow T[\sigma] \text { is a coded } \Sigma_{1}^{0} \text { tree }\right) .
$$


Since the property of being a $\Sigma_{1}^{0}$ tree can be expressed in a $\Sigma_{1}^{0}$ manner, this predicate is $\Pi_{2}^{0}$. Similarly, the predicate which says $T[\tau]$ is an $n$-uncoded $\Pi_{2}^{0}$ tree (for a fixed value of $n$ ) is $\Sigma_{2}^{0}$. Formally, $T[\tau]$ is an $n$-uncoded $\Pi_{2}^{0}$ tree if and only if there are disjoint $\tau_{0}, \ldots, \tau_{n-1}$ such that $\tau \prec \tau_{i}$ and $T\left[\tau_{i}\right]$ is a coded $\Sigma_{1}^{0}$ tree and for all disjoint $\tau_{0}, \ldots, \tau_{n}$ such that $\tau \prec \tau_{i}$, at least one $\tau_{i}$ is not the root of a coded $\Sigma_{1}^{0}$ tree. Since expressing $T[\sigma]$ is a coded $\Sigma_{1}^{0}$ tree is a $\Sigma_{1}^{0}$ statement, this entire expression is the conjunction of a $\Sigma_{1}^{0}$ and a $\Pi_{1}^{0}$ statement, and hence is $\Sigma_{2}^{0}$.

Assume that $T$ is a computable tree which is isomorphic to $T_{P}$. By the comments above, $0^{\prime \prime}$ can determine which nodes at level 1 in $T_{P}$ and $T$ are the base of coded $\Pi_{2}^{0}$ trees and which are the base of $n$-uncoded $\Pi_{2}^{0}$ trees. Once we match these nodes up, we can use $0^{\prime}$ to build the isomorphism above level 1, since we are essentially back in the case of $\Sigma_{1}^{0}$ trees.

Because we can describe the isomorphism type of $T_{P}$ precisely, we can build a computable tree $S_{P} \subseteq \omega^{<\omega}$ which is isomorphic to $T_{P}$ and for which

$$
A=\left\{x \mid S_{P}[\langle x\rangle] \text { is a coded } \Pi_{2}^{0} \text { tree }\right\}
$$

is computable. To see that $T_{P}$ need not be $\Delta_{2}^{0}$-categorical, consider the case when $P(x)$ is $\Pi_{2}^{0}$-complete. If $f: T_{P} \rightarrow S_{P}$ is an isomorphism, then $P(x) \Leftrightarrow f(x) \in A$. Therefore, $f$ cannot be $\Delta_{2}^{0}$ without contradicting the $\Pi_{2}^{0}$-completeness of $P$.

The last example we consider before the general case is how to code the $\Sigma_{3}^{0}$ relation

$$
P(x) \Leftrightarrow \exists b \forall c \exists d R(x, b, c, d) .
$$

We first code $P(x)$ into a computable tree $T_{P} \subseteq \omega^{<\omega}$ as follows.

1. $T_{P}$ is $\omega$-branching at the root. In this case, we let $\langle n\rangle \in T_{P}$ for all $n$.

2. For each $n$ : $T_{P}[\langle n\rangle]$ is $\omega$-branching at the root; for each $m, T_{P}[\langle n\rangle]$ has infinitely many nodes at level 1 each of which is the root of the tree for the $\Pi_{2}^{0}$ sentence $\forall c \exists d R(n, m, c, d)$; and for each $m, T_{P}[\langle n\rangle]$ has infinitely many nodes at level 1 each of which is the root of the $m$-uncoded $\Pi_{2}^{0}$ tree.

If $P(n)$ holds, then $T_{P}[\langle n\rangle]$ consists of infinitely many coded $\Pi_{2}^{0}$ trees as well as infinitely many $m$-uncoded $\Pi_{2}^{0}$ trees for each $m$. We refer to any tree with this isomorphism type as a coded $\Sigma_{3}^{0}$ tree. If $P(n)$ does not hold, then $T_{P}[\langle n\rangle]$ consists of infinitely many $m$-uncoded $\Pi_{2}^{0}$ trees for each $m$, and nothing else. We refer to any tree with this isomorphism type as an uncoded $\Sigma_{3}^{0}$ tree. As above, we are coding the $\Sigma_{3}^{0}$ sentences $P(n)$ by effectively constructing a tree $T_{P}[\langle n\rangle]$ which is a coded $\Sigma_{3}^{0}$ tree if the $\Sigma_{3}^{0}$ sentence $P(n)$ is true and is an uncoded $\Sigma_{3}^{0}$ tree if the $\Sigma_{3}^{0}$ sentence $P(n)$ is false.

We can describe the isomorphism type of $T_{P}$ precisely as follows. $T_{P}$ is $\omega$-branching at the root and consists of infinitely many coded $\Sigma_{3}^{0}$ trees and infinitely many uncoded $\Sigma_{3}^{0}$ trees.

To see that $T_{P}$ is $\Delta_{4}^{0}$-categorical, let $T$ be any computable tree which is isomorphic to $T_{P}$. For any $\tau \in T$ at level $1, T[\tau]$ is a coded $\Sigma_{3}^{0}$ tree if and only if

$$
\exists \tau_{2}\left(\tau<\tau_{2} \wedge \operatorname{level}\left(\tau_{2}\right)=2 \wedge T\left[\tau_{2}\right] \text { is a coded } \Pi_{2}^{0} \text { tree }\right) .
$$


Since determining if $T\left[\tau_{2}\right]$ is a coded $\Pi_{2}^{0}$ tree is $\Pi_{2}^{0}$, this predicate is $\Sigma_{3}^{0}$. Therefore, $0^{\prime \prime \prime}$ can determine which nodes at level 1 in $T$ and $T_{P}$ are the base of a coded $\Sigma_{3}^{0}$ tree and which are the base of an uncoded $\Sigma_{3}^{0}$ tree. Once these nodes are matched up correctly, $0^{\prime \prime}$ can build the rest of the isomorphism as in the previous case. Therefore, $T_{P}$ is $\Delta_{4}^{0}$-categorical.

Since we can describe the isomorphism type of $T_{P}$ exactly, we can build a computable tree $S_{P} \subseteq \omega^{<\omega}$ which is isomorphic to $T_{P}$ and such that

$$
A=\left\{n \mid S_{P}[\langle n\rangle] \text { is a coded } \Sigma_{3}^{0} \text { tree }\right\}
$$

is computable. As above, if $P(x)$ is $\Sigma_{3}^{0}$-complete, there cannot be a $\Delta_{3}^{0}$ isomorphism between $T_{P}$ and $S_{P}$.

We now present two general constructions. First, we use a construction similar to the $\Pi_{2}^{0}$ coding to pass from a $\Sigma_{n}^{0}$ coding to a $\Pi_{n+1}^{0}$ coding. Let $P(x)$ be an infinite and coinfinite $\Pi_{n+1}^{0}$ relation such that

$$
P(x) \Leftrightarrow \forall a R(x, a)
$$

where $R$ is $\Sigma_{n}^{0}$. Assume that we have defined the isomorphism types for a coded $\Sigma_{n}^{0}$ tree and an uncoded $\Sigma_{n}^{0}$ tree and that such trees are not isomorphic. Assume that these trees are defined in such a way that given any $\Sigma_{n}^{0}$ sentence we can effectively construct a tree which is a coded $\Sigma_{n}^{0}$ tree if the sentence is true and is an uncoded $\Sigma_{n}^{0}$ tree if the sentence is false. Furthermore, assume that there is a $\Sigma_{n}^{0}$ sentence which is true in the coded $\Sigma_{n}^{0}$ tree and false in the uncoded $\Sigma_{n}^{0}$ tree. We define $T_{P}$ as the closure of the following conditions under initial segments.

1. $T_{P}$ is $\omega$-branching at the root. Formally, we put $\langle 1\rangle \in T_{P}$ and $\left\langle p^{u}\right\rangle \in T_{P}$ for all primes $p$ and $u \geq 1$.

2. For all $u$ and $m, T_{P}\left[\left\langle 2^{u}, m\right\rangle\right]$ is constructed to code the $\Sigma_{n}^{0}$ sentence $\forall a \leq m R(u, a)$.

3. For all odd primes $p$ and $u \geq 1, T_{P}\left[\left\langle p^{u}\right\rangle\right]$ consists of $(u-1)$ copies of the coded $\Sigma_{n}^{0}$ tree and infinitely many copies of the uncoded $\Sigma_{n}^{0}$ tree.

If $P(u)$ holds, then the root of $T_{P}\left[\left\langle 2^{u}\right\rangle\right]$ is $\omega$-branching and the nodes at level 1 in this tree are all roots of coded $\Sigma_{n}^{0}$ trees. We refer to any tree with this isomorphism type as a coded $\Pi_{n+1}^{0}$ tree.

If $P(u)$ does not hold, then the root of $T_{P}\left[\left\langle 2^{u}\right\rangle\right]$ is $\omega$-branching, and the trees above the nodes at level 1 contain infinitely many copies of the uncoded $\Sigma_{n}^{0}$ tree and for some $m$, exactly $m$ copies of the coded $\Sigma_{n}^{0}$ tree. We refer to any tree with this isomorphism type as an $m$-uncoded $\Pi_{n+1}^{0}$ tree.

The isomorphism type of $T_{P}$ is uniquely determined by the fact that the root is $\omega$-branching and $T_{P}$ consists of infinitely many copies of the coded $\Pi_{n+1}^{0}$ tree and infinitely many copies of the $m$-uncoded $\Pi_{n+1}^{0}$ tree for each $m$.

For any computable tree $T$ isomorphic to $T_{P}$ and any $\tau \in T$ at level $1, T[\tau]$ is a coded $\Pi_{n+1}^{0}$ tree if for all $m$, there are distinct nodes $\tau_{0}, \ldots, \tau_{m}$ such that for all $i<m$,

$$
\tau<\tau_{i} \wedge \operatorname{level}\left(\tau_{i}\right)=2 \wedge T_{P}\left[\tau_{i}\right] \text { is a coded } \Sigma_{n}^{0} \text { tree. }
$$


This condition is $\Pi_{n+1}^{0}$ by our assumption on the complexity of determining if a tree is a coded $\Sigma_{n}^{0}$ tree. Furthermore, $T[\tau]$ is an $m$-uncoded $\Pi_{n+1}^{0}$ tree if there exist distinct nodes $\tau_{1}, \ldots, \tau_{m}$ such that for all $1 \leq i \leq m$, equation (1) holds (for $m=0$ this check is vacuous), but for all choices of nodes $\tau_{1}, \ldots, \tau_{m+1}$, there is some $1 \leq i \leq m+1$ such that

$$
\left(\tau<\tau_{i} \wedge \operatorname{level}\left(\tau_{i}\right)=2\right) \rightarrow T_{P}\left[\tau_{i}\right] \text { is an uncoded } \Sigma_{n}^{0} \text { tree. }
$$

Altogether, this condition is the conjunction of a $\Sigma_{n}^{0}$ statement and a $\Pi_{n}^{0}$ statement.

To see that $T_{P}$ is $\Delta_{n+2}^{0}$-categorical, notice that each of the conditions in the previous paragraph can be determined by $0^{(n+1)}$. Therefore, given any computable tree $T$ isomorphic to $T_{P}, 0^{(n+1)}$ can match up the nodes at level 1 correctly. The fact that the rest of the isomorphism can be built follows by induction.

To see that $T_{p}$ is not $\Delta_{n+1}^{0}$-categorical, consider the case when $P(x)$ is $\Pi_{n+1}^{0}$-complete. Because we know the isomorphism type of $T_{P}$ exactly, we can build a computable tree $S_{P}$ such that

$$
A=\left\{m \mid S_{P}[\langle m\rangle] \text { is a coded } \Pi_{n+1}^{0} \text { tree }\right\}
$$

is computable. If $f: T_{P} \rightarrow S_{P}$ is an isomorphism, then $P(x)$ holds if and only if $f\left(\left\langle 2^{x}\right\rangle\right)=\langle m\rangle$ for some $m \in A$. Therefore, $f$ cannot be $\Delta_{n}^{0}$ without contradicting the fact that $P$ is $\Pi_{n+1^{-}}^{0}$ complete.

It remains to show how to pass from a $\Pi_{n}^{0}$ coding to a $\Sigma_{n+1}^{0}$ coding. Let $P(x)$ be an infinite and coinfinite $\Sigma_{n+1}^{0}$-complete relation given by

$$
P(x) \Leftrightarrow \exists a R(x, a)
$$

where $R$ is $\Pi_{n}^{0}$. Assume that we have determined the isomorphism types for the coded and $m$-uncoded $\Pi_{n}^{0}$ trees with the corresponding complexity results as above. We define the computable tree $T_{P}$ as the downward closure of the following conditions.

1. $T_{P}$ is $\omega$-branching at the root. Formally, for all $u,\langle u\rangle \in T_{P}$.

2. For each $u$ : $T_{P}[\langle u\rangle]$ is $\omega$-branching at the root; for each $m, T_{P}[\langle u\rangle]$ has infinitely many nodes at level 1 each of which is the root of tree coding the $\Pi_{n}^{0}$ sentence $R(u, m)$; and for each $m, T_{P}[\langle u\rangle]$ has infinitely many nodes at level 1 each of which is the root of an $m$-uncoded $\Pi_{n}^{0}$ tree.

If $P(u)$ holds, then $T_{P}[\langle u\rangle]$ is $\omega$-branching at the root and contains infinitely many copies of the coded $\Pi_{n}^{0}$ tree and infinitely many copies of the $m$-uncoded $\Pi_{n}^{0}$ tree for each $m$. We refer to any tree with this isomorphism type as a coded $\Sigma_{n+1}^{0}$ tree.

If $P(u)$ does not hold, then $T_{P}[\langle u\rangle]$ is $\omega$-branching at the root and consists of infinitely many copies of the $m$-uncoded $\Pi_{n}^{0}$ tree for each $m$. In particular, there are no coded $\Pi_{n}^{0}$ trees in $T_{P}[\langle u\rangle]$. We refer to any tree with this isomorphism type as an uncoded $\Sigma_{n+1}^{0}$ tree.

Let $T$ be a computable tree isomorphic to $T_{P}$ and suppose $\tau \in T$ is a level 1 node. $T[\tau]$ is a $\Sigma_{n+1}^{0}$ coded tree if and only if there is a $\tau_{2} \in T$ such that

$$
\tau<\tau_{2} \wedge \operatorname{level}\left(\tau_{2}\right)=2 \wedge T\left[\tau_{2}\right] \text { is a coded } \Pi_{n}^{0} \text { tree. }
$$


By assumption on the complexity of $\Pi_{n}^{0}$ trees, this condition is $\Sigma_{n+1}^{0}$. As above, this condition implies that $T_{P}$ is $\Delta_{n+2}^{0}$-categorical. To show that $T_{P}$ is not $\Delta_{n+1}^{0}$-categorical, we define a tree $S_{P}$ and argue as above.

\section{Trees Under the Infimum Function}

We end this paper with a brief discussion about trees defined using the infimum function and a conjecture about when they are computably categorical. We have already mentioned (see Section 1) that if $(T, \wedge)$ is computable, then the corresponding $(T, \prec)$ is also computable. However, it is simple to build a tree $T$ in which $\prec$ is computable and $\wedge$ is not. Start with 0 as the root, and make every even number a successor of 0 at level 1 . To diagonalize against the possibility that $\varphi_{e}$ computes $\wedge$, we wait until $\varphi_{e}(\langle 4 e+2,4 e+4\rangle) \downarrow=0$, and if this ever happens, we add the next available odd number $x$ to $T$ at level 1 with $x \prec 4 e+2$ and $x \prec 4 e+4$. $T$ will have domain $\omega$ and height 3 , and $\prec$ will be computable, but our diagonalization ensures that $\wedge$ is not computable.

The notion of an embedding depends strongly on whether we define trees using $\prec$ or $\wedge$. Consider the following two trees:

It is easy to embed $I_{0}$ into $I_{1}$ with respect to $\prec$, but there is no embedding of $I_{0}$ into $I_{1}$ respecting $\wedge$. (The infimum of any pair of distinct nodes in $I_{0}$ is the root, whereas no possible image of $I_{0}$ in $I_{1}$ has the same property.) For the remainder of this section, therefore, we will speak of $\prec$-embeddings and $\wedge$-embeddings, to distinguish these two types of embeddings. In the rest of the paper, of course, "embedding" always means $\prec$-embedding. Notice that for an isomorphism, it does not matter which notion we use. That is, any isomorphism between trees under $\prec$ is also an isomorphism of the same trees under $\wedge$ (and conversely).

The simple example above creates problems when one investigates computable categoricity. Consider the tree $T$ which consists of a root with infinitely many copies of $I_{0}$ and infinitely many copies of $I_{1}$ (and nothing else) as successor trees above the root. This tree has height 4, and $(T, \prec)$ is not of finite type, hence not computably categorical, by Theorem 1.8. However, $(T, \wedge)$ is computably categorical. Clearly we can build a computable copy of $(T, \wedge)$, and given any two computable copies, we can find the root of each, then identify successor trees of each type in each copy and match them up. In particular, every successor tree contains three pairwise-incomparable nodes $a, b$, and $c$, and once those nodes have appeared, we simply compute $a \wedge b, a \wedge c$, and $b \wedge c$. The successor tree is of type $I_{0}$ iff these three infima are equal.

We do have the following result. 
Lemma 6.1 Any computably categorical tree $(T, \prec)$ will still be computably categorical when re-interpreted as $(T, \wedge)$, assuming only that the function $\wedge$ so defined is computable.

Proof. If a computable structure $\left(T^{\prime}, \wedge^{\prime}\right)$ is isomorphic to $(T, \wedge)$, then the corresponding $\left(T^{\prime}, \prec^{\prime}\right)$ is also computable, hence isomorphic to $(T, \prec)$ via some computable $\varphi$. As noted above, the isomorphism $\varphi$ must also preserve infima, so it is an isomorphism of $(T, \wedge)$ onto $\left(T^{\prime}, \wedge^{\prime}\right)$ as required.

To determine computable categoricity for trees under the infimum function, therefore, we need to consider $\wedge$-embeddings rather than $\prec$-embeddings. Fortunately, one of our main tools, Kruskal's Theorem (stated as Theorem 3.4), yields not only $\prec$-embeddings but also $\wedge$-embeddings. The first step, therefore, is to refine the notion of being of finite type by referring to $\wedge$ instead of $\prec$.

Definition 6.2 1. A tree $T$ is of strongly finite $\wedge$-type if it satisfies Definition 1.6 when the word "embedding" is replaced everywhere by " $\wedge$-embedding."

2. A tree $T$ is of finite $\wedge$-type if it satisfies Definition 1.7 when the word "embedding" is replaced everywhere by " $\wedge$-embedding" and "strongly finite type" is replaced everywhere by "strongly finite $\wedge$-type."

Notice that in our example from the previous page, $(T, \wedge)$ has finite $\wedge$-type but $(T, \prec)$ does not have finite $\prec$-type.

We conjecture that with these definitions, the proofs from Sections 2, 3, and 4 will go through with relatively few modifications, as long as one always refers to $\wedge$-embeddings and (strongly) finite $\wedge$-type. Thus we would have the analogue of Theorem 1.8:

Conjecture 6.3 For a computable tree $(T, \wedge)$ of finite height, the following are equivalent:

1. $T$ is of finite $\wedge$-type;

2. $(T, \wedge)$ is computably categorical;

3. $(T, \wedge)$ has finite computable dimension;

4. $(T, \wedge)$ is relatively computably categorical.

In [24], Miller proved the corresponding result for computable trees $(T, \wedge)$ of infinite height: the computable dimension of $(T, \wedge)$ must be $\omega$. Together with Conjecture 6.3, this would answer the question of computable categoricity for all trees under the infimum function. 


\section{References}

[1] C.J. Ash; Categoricity in hyperarithmetical degrees, Annals of Pure and Applied Logic 34 (1987), 1-14.

[2] C.J. Ash \& J.F. Knight; Computable Structures and the Hyperarithmetic Hierarchy (Amsterdam: Elsivier Science 2000.)

[3] C. J. Ash, J. F. Knight, M. Mannasse, \& T. Slaman; Generic copies of countable structures, Annals of Pure and Applied Logic 42 (1989), 195-205.

[4] J. Chisholm; On intrisically 1-computable trees, unpublished manuscript.

[5] J.N. Crossley, A.B. Manaster, \& M.F. Moses; Recursive categoricity and recursive stability, Annals of Pure and Applied Logic 31 (1986), 191-204.

[6] R.G. Downey; On presentations of algebraic structures, in Complexity, Logic, and Recursion Theory, ed. A. Sorbi (New York: Dekker, 1997), 157-205.

[7] R.G. Downey \& C.G. Jockusch; Every low Boolean algebra is isomorphic to a recursive one, Proceedings of the American Mathematical Society 122 (1994), 871-880.

[8] S.S. Goncharov; Autostability and computable families of constructivizations, Algebra and Logic 14 (1975), 647-680 (Russian), 392-409 (English translation).

[9] S.S. Goncharov; Autostable models and algorithmic dimensions, Handbook of Recursive Mathematics, vol. 1 (Amsterdam: Elsevier, 1998), 261-287.

[10] S.S. Goncharov; Nonequivalent constructivizations, Proc. Math. Inst. Sib. Branch Acad. Sci. (Novosibirsk: Nauka, 1982).

[11] S.S. Goncharov; The problem of the number of non-self-equivalent constructivizations, Algebra and Logic 19 (1980), 401-414 (English translation).

[12] S.S. Goncharov; Groups with a finite number of constructivizations, Soviet Math. Dokl. 19 (1981) 58-61.

[13] S.S. Goncharov \& V.D. Dzgoev; Autostability of models, Algebra and Logic 19 (1980), 45-58 (Russian), 28-37 (English translation).

[14] S.S. Goncharov, S. Lempp \& R. Solomon; The computable dimension of ordered abelian groups, to appear in Advances in Mathematics.

[15] S.S. Goncharov, A.V. Molokov \& N.S. Romanovskii; Nilpotent groups of finite algorithmic dimension, Siberian Math Journal 30 (1989), 63-68. 
[16] D.R. Hirschfeldt, B. Khoussainov, R.A. Shore, \& A.M. Slinko; Degree spectra and computable dimension in algebraic structures, Annals of Pure and Applied Logic 115 (2002), 71-113.

[17] B. Khoussainov \& R.A. Shore; Computable isomorphisms, degree spectra of relations, and Scott families, Annals of Pure and Applied Logic 93 (1998), 153-193.

[18] B. Khoussainov \& R.A. Shore; Effective model theory: the number of models and their complexity, Models And Computability: Invited Papers from Logic Colloquium '97, ed. S.B. Cooper \& J.K. Truss, LMSLNS 259 (Cambridge: Cambridge University Press, 1999), 193-240.

[19] J.B. Kruskal; Well quasi-ordering, the tree theorem, and Vázsonyi's conjecture, Transactions of the American Mathematical Society 95 (1960), 210-225.

[20] O.V. Kudinov; An autostable 1-decidable model without a computable Scott family of $\exists$ formulas, Algebra and Logic 35 (1996), 255-260 (English translation).

[21] O.V. Kudinov; An integral domain with finite algorithmic dimension, unpublished manuscript.

[22] P. LaRoche, Recursively presented Boolean algebras, Notices Amer. Math. Soc. 24 (1977), A-552, research announcement.

[23] G. Metakides \& A. Nerode; Effective content of field theory, Ann. Math. Logic 17 (1979), 289-320.

[24] R.G. Miller; The computable dimension of trees of infinite height, to appear in Journal of Symbolic Logic.

[25] R.G. Miller; The $\Delta_{2}^{0}$ spectrum of a linear order, Journal of Symbolic Logic 66 (2001), 470-486.

[26] C.St.J.A. Nash-Williams; On well-quasi-ordering finite trees, Proc. Cambridge Phil. Soc. 59 (1963), 833-835.

[27] A.T. Nurtazin; Computable classes and algebraic criteria of autostability, thesis from Math. Inst. Siberian Branch of SSSR Acad. Sci., Novosibirsk, 1974 (Russian).

[28] A.T. Nurtazin; Strong and weak constructivizations and enumerable families, Algebra and Logic 13 (1974), 177-184.

[29] J.B. Remmel; Recursively categorical linear orderings, Proceedings of the American Mathematical Society 83 (1981), 387-391.

[30] J.B. Remmel; Recursive isomorphism types of recursive Boolean algebras, Journal of Symbolic Logic 46 (1981), 572-594. 
[31] S.G. Simpson; Nonprovability of certain combinatorial properties of finite trees, Harvey Friedman's Research on the Foundations of Mathematics, ed. L. A. Harrington, M. D. Morley, A. Scedrov \& S. G. Simpson (Amsterdam: North-Holland, 1985), 87117.

[32] T.A. Slaman; Relative to any nonrecursive set, Proceedings of the American Mathematical Society 126 (1998), 2117-2122.

[33] R.I. Soare; Recursively Enumerable Sets and Degrees (New York: Springer-Verlag, 1987).

[34] S. Wehner; Enumerations, countable structures, and Turing degrees, Proceedings of the American Mathematical Society 126 (1998), 2131-2139.

[35] W. White, On the complexity of categoricity in computable structures, Mathematical Logic Quarterly 496 (2003), 603-614.

\author{
Department of Mathematics \\ UNIVERSITY OF WISCONSIN \\ 480 LinCOLN DRIVE \\ MADISON, WI 53706-1388 \\ E-mail: lempp@math.wisc.edu \\ E-mail: cmccoy1@nd.edu \\ Department of Mathematics \\ Queens College - C.U.N.Y. \\ 65-30 Kissena Blvd. \\ Flushing, New York 11367 \\ E-mail:rmiller@forbin.qc.edu \\ 196 Auditorium RoAd \\ University of Connecticut U-3009 \\ Department of Mathematics \\ STORRs, CT 06269-3009 \\ E-mail: solomon@math.uconn.edu
}

